\title{
A Bio-inspired Excitable Cardiac Cell Membrane from Electroactive Polymers: Ion Transport Studies
}

A Dissertation Presented to the

Faculty of the School of Engineering and Applied Science

University of Virginia

In Partial Fulfillment

of the Requirements for the Degree of

Doctor of Philosophy

Mechanical and Aerospace Engineering

Christina Viau Haden Moored

December 2012 


\section{APPROVAL SHEET}

The dissertation is submitted in partial fulfillment of the requirements for the degree of

Doctor of Philosophy in Mechanical and Aerospace Engineering

Christina Viau Haden Moored

The dissertation has been read and approved by the examining committee:

Pamela M. Norris, Advisor

Silvia Blemker, Chairman

Hilary Bart-Smith

Edward Berger

Donald A. Jordan

John Scully

Accepted for the School of Engineering and Applied Science:

James H. Aylor, Dean

School of Engineering and Applied Science

December 2012 


\begin{abstract}
The heart is an incredibly efficient biological pump, which contracts as a whole from the coordinated effort of millions of excitable cells called cardiac myocytes. These cells are considered excitable since they possess the ability to respond to a threshold activation signal, but only if sufficient time has passed and they have recovered from their previous excitation. Excitable cells also have the capacity to propagate an activation signal to their neighboring cells, and collectively are referred to as an excitable medium. Numerous examples of excitable media exist in nature, including the heart, forest fires, the intestine, nerves, and certain kinds of chemical reactions. Engineered artificial excitable media, however, have never been developed before.

The long-term goal of this project, therefore, is to develop the first such artificial excitable medium from an array of artificial excitable cells, called gel-cells. The conceptual design of the gel-cell consists of a thin electroactive ion-gating membrane (polypyrrole), sandwiched between two layers of hydrated polymer (hydrogel) loaded with different concentrations of potassium chloride $\left(\mathrm{KCl}_{a q}\right)$ electrolytes. The voltage controlled artificial membrane initially confines ions to the upper chamber of the gelcell, and releases them upon membrane opening into the lower chamber, a process similar to membrane activation in cardiac myocytes. Potassium sensing electrodes embedded throughout the gel-cell provide feedback on the location and concentration of ions within the gel-cell, becoming markers for its activation. The scope of the research presented here was to construct the biomimetic cell membrane portion of the gel-cell, and study ion transport through its assembly.

First, the electrochemical response of polypyrrole (PPy) was characterized via cyclic voltammetry, and its membrane morphology and thickness were observed via scanning electron microscopy. Experimentally obtained membrane thickness values were then compared to model predictions, which were subsequently improved by updating membrane density and structure assumptions. Hydrogel pore size approximations were correlated to equilibrium water content calculations, and $\mathrm{KCl}_{a q}$ concentrations were quantified using electrochemical impedance spectroscopy. Time-resolved $\mathrm{KCl}_{a q}$ flux experiments were performed on PPy membranes in solution, providing real-time concentration profiles of $\mathrm{KCl}_{a q}$ as it passes through the membrane. From the parameter space tested, the optimal membrane preparation and gating potentials were selected which produced the highest flux rates. Time-lapse flux experiments (providing piece-wise concentration profiles for $\mathrm{KCl}_{a q}$ ) were then performed on PPy membranes in both solution and hydrogel. Ion transport through two nearly identical membranes produced under different deposition conditions $\left(\mathrm{PPy}_{a}\right.$ and $\left.\mathrm{PPy}_{b}\right)$ were compared in solution. The slightly denser and thicker membrane $\left(\mathrm{PPy}_{b}\right)$ produced nearly an order of magnitude higher flux rates than $\mathrm{PPy}_{a}$, suggesting that an active component of ion transport occurs predominantly through these membranes via binding and release mechanisms in PPy. Next, experiments performed on PPy membranes in hydrogel demonstrated that flux rates through these assemblies were proportional to hydrogel pore size. In addition, ion transport was reduced by two
\end{abstract}


orders of magnitude through PPy/hydrogel as compared to PPy in solution. Finally, the potentiometric response of potassium sensing electrodes was quantified in novel configurations of hydrogel versus hydrogel, demonstrating their ability to observe relative $\mathrm{KCl}_{a q}$ concentrations in this material. These results lay the foundation for the development of the gel-cell by providing a cardiac myocyte membrane analog made from electroactive polymer materials, and identify key variables which can be varied to optimize ion transport in these assemblies. 
I dedicate this work to my parents, Louise Viau and Timothy Wash Haden Jr., who always encouraged me to pursue my dreams. 


\section{Contents}

Acknowledgements viii

1 Introduction 1

2 Background $\quad 6$

2.1 Cardiac Myocytes and the Heart . . . . . . . . . . . 6

2.2 A Biomimetic Heart Cell . . . . . . . . . . . . . . . . . . . . . 13

2.2.1 Operating Sequence for an Excitable Gel-cell . . . . . . . . . . 15

2.2.2 Biomimetic Cell Components . . . . . . . . . . . . . . . 17

2.2.2.1 Polypyrrole . . . . . . . . . . . . . . . 18

2.2.2.2 Hydrogels . . . . . . . . . . . . . . . . . . . . 20

2.2.2.2.1 Solute Diffusion . . . . . . . . . . . . 23

2.2.2.2.2 Biocompatibility . . . . . . . . . . 23

2.2.2.3 Ion Selective Electrode . . . . . . . . . . . . . 24

2.2.2.4 Ionic Switch . . . . . . . . . . . . . . . 25

2.2.3 Potential Applications of the Artificial Cell . . . . . . . . . 25

3 Methods 28

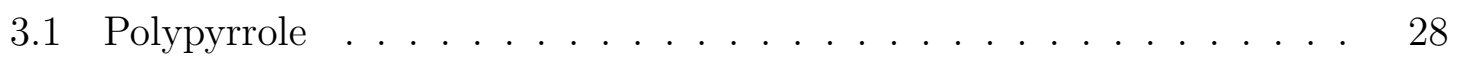

3.1.1 Polypyrrole for Flux Experiments . . . . . . . . . . . . . . . 29

3.1.1.1 Materials . . . . . . . . . . . . . . . . 29

3.1.1.2 Preparation . . . . . . . . . . . . . . . . 29

3.1.2 Polypyrrole for Ion Selective Electrodes . . . . . . . . . . . . . 31

3.1.2.1 Materials . . . . . . . . . . . . . . . 31

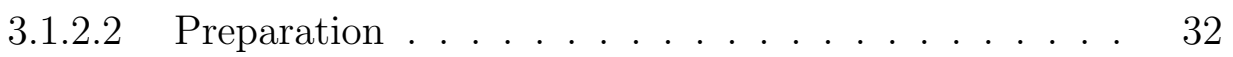

3.2 PPy Thickness . . . . . . . . . . . . . . . . . . . . . . . . . . . . . . . . . . . . . .

3.2.1 Theoretical Prediction . . . . . . . . . . . . . . 33

3.2.2 Scanning Electron Microscopy . . . . . . . . . . . . . 34

3.3 Hydrogel . . . . . . . . . . . . . . . . . . . . . . . . . . . . . . . . . . . . . . . . 35

3.3.1 Materials ..................... 35

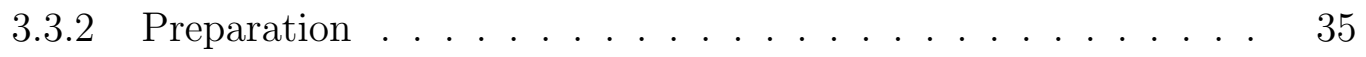

3.4 Potassium Chloride Detection . . . . . . . . . . . . . . 36

3.4.1 Electrochemical Impedance Spectroscopy . . . . . . . . . . . . 36

3.4.1.1 Materials and Preparation ........... 36

3.4 .2 Ion Selective Electrode . . . . . . . . . . . . . . . . . . 39 
3.4.2.1 Materials and Preparation . . . . . . . . . . . 39

3.5 Flux Rate Measurements . . . . . . . . . . . . . . . . . . . . 43

3.5.1 Flux Cell ........................ 43

3.5.2 'Time-Resolved' versus 'Time-Lapse' Measurements . . . . . . 46

3.5.3 Flux through PPy / Hydrogel . . . . . . . . . . . . . . . . . 48

4 Results $\quad 50$

4.1 Polypyrrole ........................ . . . 50

4.1 .1 Cyclic Voltammetry . . . . . . . . . . . . 51

4.1.2 Scanning Electron Microscopy . . . . . . . . . . . . . . 53

4.1.3 Thickness Determination and Prediction ........... . 54

4.1.4 Comparable Membranes from Different Deposition Schemes . 61

4.2 Hydrogel . . . . . . . . . . . . . . . . . . . 63

4.2.1 Water Content Determination . . . . . . . . . . . . . 63

4.2.2 Electrochemical Impedance Spectroscopy . . . . . . . . . . . . 65

4.3 Flux Experiments . . . . . . . . . . . . . . . . . . . 67

4.3.1 Time-Resolved Concentration Readings . . . . . . . . . . . . . 67

4.3.1.1 Membrane Activation Parameters . . . . . . . . . . 70

4.3.1.2 Membrane Performance Variability . . . . . . . . . . 72

4.3.2 Time-Lapse Concentration Readings in Solution . . . . . . . . 77

4.3.2.1 Comparing Membrane Deposition Schemes . . . . . . 78

4.3.2.2 Comparing Concentration Gradients . . . . . . . . . 80

4.3.3 Time-Lapse Concentration Readings in PPy / Hydrogel . . . . 81

4.4 Potassium Ion Selective Electrode . . . . . . . . . . . . . . . . 85

5 Discussion $\quad 89$

5.1 Polypyrrole as an Artificial Cell Membrane . . . . . . . . . . . . . 89

5.1.1 Ion Gating . . . . . . . . . . . . . . . . . . . . 90

5.1.2 Active Ion Pumping through Cycling . . . . . . . . . . . . 91

5.2 Hydrogel as Artificial Intra- and Extracellular Space . . . . . . . . . . 95

5.2.1 Hydrogel Water Content . . . . . . . . . . . . . . . . . 95

5.2.2 Solute Diffusivity in Hydrogel . . . . . . . . . . . . . . . . . 97

6 Conclusions and Future Work $\quad 99$

6.1 Summary of Results . . . . . . . . . . . . . . . . . . . . . . 99

6.1.1 Polypyrrole and Hydrogel . . . . . . . . . . . . . . . . . 100

6.1.2 Flux Experiments . . . . . . . . . . . . . . . . . 101

6.1.3 Ion Selective Electrode Studies . . . . . . . . . . . . . . . . . 104

6.2 Recommendations for Future Work . . . . . . . . . . . . . . . 105

6.2.1 Excitability and Refractoriness . . . . . . . . . . . 105

6.2.2 Connecting Gel-Cells . . . . . . . . . . . . . . . . . 105

6.2.3 Cell Contractility . . . . . . . . . . . . . . 106

6.2 .4 Component Improvement . . . . . . . . . . . . . . . . . . . . 106

6.2.5 Miniaturization . . . . . . . . . . . . . . . 107 
A Summary of Failed Experiments $\quad 126$

A.1 Ionic Liquid Polymer Gels . . . . . . . . . . . . . . . . . . . . 126

A.1.1 Mass Spectroscopy . . . . . . . . . . . . . . . . . 127

A.1.2 Ultraviolet-Visible Spectroscopy . . . . . . . . . . . . . . . . . 127

A.1.3 Mohr Method . . . . . . . . . . . . . . . . . . . 128

A.1.4 EIS Probe and Ion Selective Electrodes . . . . . . . . . . . . . 129

A.1.5 Conclusion . . . . . . . . . . . . . . . . . 130

A.2 Working Electrode Material . . . . . . . . . . . . . . . 130

A.2.1 Stainless Steel Mesh . . . . . . . . . . . . . . . . . 130

A.2.2 Gold Plated Stainless Steel Mesh . . . . . . . . . . . . . . 131

A.2.3 Gold Mesh . . . . . . . . . . . . . . . . . . . . . . . . . 133

A.2.4 Conclusion . . . . . . . . . . . . . . . . . 133

A.3 Time-Resolved Hydrogel Flux Experiments . . . . . . . . . . . . . . . 134 


\section{List of Figures}

1.1 Human heart inspired artificial excitable cell. . . . . . . . . . . . . . 2

2.1 Microscopy images of cardiac myocytes. . . . . . . . . . . . . . . 7

2.2 Voltage-gated ion channels schematic. . . . . . . . . . . . 8

2.3 Action potential cycle of the cardiac myocyte. . . . . . . . . . . . . 9

2.4 Cardiac wave propagation example. . . . . . . . . . . . . . 10

2.5 Conceptual design of the artificial excitable cell. . . . . . . . . . . . . 14

2.6 Sequence diagram of the artificial excitable cell cycle. . . . . . . . . . 16

2.7 Chemical structure of pyrrole. . . . . . . . . . . . . . . . . . 18

2.8 Microscopy images of polypyrrole. . . . . . . . . . . . . . . . . 19

2.9 Illustration of the oxidation and reduction of PPy. . . . . . . . . . 20

2.10 Voltammetric response for an ion selective electrode. . . . . . . . . . 25

2.11 Potential applications for the artificial cell. . . . . . . . . . . . . 27

3.1 Schematic of a 3-electrode electrochemical cell. . . . . . . . . . . . . . 30

3.2 PPy on stainless steel plate. . . . . . . . . . . . . . . . 31

$3.3 \mathrm{PPy} / \mathrm{CH}_{3} \mathrm{CN}$ on gold mesh, before and after. . . . . . . . . . . . . . 32

$3.4 \mathrm{PPy} / \mathrm{diH}_{2} \mathrm{O}$ thickness determination via SEM. . . . . . . . . . . . . 34

3.5 Electrochemical impedance spectroscopy probe. . . . . . . . . . . 37

3.6 EIS probe equivalent electrical circuit. . . . . . . . . . . . . . . . . . . . . . . . . 38

3.7 EIS probe response in $\mathrm{KCl}_{a q} \ldots \ldots \ldots$. . . . . . . . . . . . . . . . . 39

3.8 Ion selective electrode testing setup. . . . . . . . . . . . . . 41

3.9 Ion selective electrodes in hydrogel. . . . . . . . . . . . . . . . . . 42

3.10 Ion selective electrode voltammetric response in hydrogel. . . . . . . . 42

3.11 Schematic of lux cell layout. . . . . . . . . . . . . . . . . . . 44

3.12 Platinum ring working electrdode Solidworks schematic. . . . . . . . . 44

3.13 Flux cell Solidworks schematic. . . . . . . . . . . . . . . 45

3.14 Flux cell layout picture. . . . . . . . . . . . . . . . . . 46

3.15 Flux experiment sequence diagrams. . . . . . . . . . . . . . . . . . 47

3.16 PPy/Hydrogel artificial cell membrane. . . . . . . . . . . . . . . . . . 48

$3.17 \mathrm{PPy} /$ Hydrogel in a flux cell. . . . . . . . . . . . . . . . . 49

4.1 Cyclic voltammetry on PPy. . . . . . . . . . . . . . . . 51

4.2 Scanning electron microscopy of PPy. . . . . . . . . . . . . . 54

4.3 Membrane thickness: prediction vs. experimental values. . . . . . . . 55

4.4 Membrane density: experimental values. . . . . . . . . . . . . . . . 57 
4.5 Membrane thickness model prediction with experimental density correction. . . . . . . . . . . . . . . . . . . 58

4.6 Membrane thickness model prediction with experimental density and y value corrections. . . . . . . . . . . . . . . . . . . . . 59

4.7 Farrington model percent error. . . . . . . . . . . . . . . . . . . 60

$4.8 \mathrm{PPy}_{a} \& \mathrm{PPy}_{b}$ experimental thickness values. . . . . . . . . . . 61

4.9 Membrane density: experimental values. . . . . . . . . . . . 62

4.10 Equilibrium water content of hydrogels. . . . . . . . . . . . . . 64

4.11 EIS probe calibration curves. . . . . . . . . . . . . . 66

4.12 Time-resolved $\mathrm{KCl}_{a q}$ concentration profile: transport initiation delay. 69

4.13 Flux rates of $\mathrm{KCl}_{a q}$ as a function of cycling pulse widths. . . . . . 72

4.14 Membrane performance variability: day-to-day. . . . . . . . . 74

4.15 Membrane performance variability: after reuse. . . . . . . . 76

4.16 Time-lapse $\mathrm{KCl}_{a q}$ concentration profile in solution. . . . . . . . . 78

4.17 Flux rates of $\mathrm{KCl}_{a q}$ as a function of membrane density. . . . . . . 80

$4.18 \mathrm{PPy} /$ hydrogel sandwich structure. . . . . . . . . . . . . . . 82

4.19 Time-lapse $\mathrm{KCl}_{a q}$ concentration profile in hydrogel. . . . . . . . . 83

4.20 Flux rates of $\mathrm{KCl}_{a q}$ as a function of hydrogel pore size. . . . . . . . 84

4.21 Schematic of ISE probes across membrane. . . . . . . . . . . 86

4.22 Hydrogel covered ISE probes. . . . . . . . . . . . . . . . 87

4.23 Potentiometric response of ISE/Hydrogel vs. ISE/Hydrogel. . . . . . 88

5.1 Schematic of ion pumping through PPy. . . . . . . . . . . 92

5.2 Schematic of PPy structure. . . . . . . . . . . . . . . . . 93

A.1 ILPG swelling . . . . . . . . . . . . . . . . . . . . . 126

A.2 Ionic conductivity of ILPG versus polymer content $\ldots \ldots \ldots \ldots$

A.3 UV Vis of ILPG . . . . . . . . . . . . . . . . . . . . . . . 128

A.4 Mohr Method . . . . . . . . . . . . . . . . . . . . . . . . 129

A.5 Chronoamperometric Response of PPy on S.S. Mesh _. . . . . . 131

A.6 Gold Plating on S.S. Illustration . . . . . . . . . . . . . . . . 132

A.7 Gold Plated S.S. Meshes, Before and After Reduction . . . . . . . . 132

A.8 PPy $/ \mathrm{CH}_{3} \mathrm{CN}$ on gold mesh, before and after . . . . . . . . 133

A.9 Flux experiment sequence diagrams. . . . . . . . . . . . . 135 


\section{List of Tables}

3.1 Hydrogel Composition Ratios with Resulting Pore Sizes . . . . . . . . 36

$4.1 \mathrm{PPy}_{a}$ and $\mathrm{PPy}_{b}:$ Membrane Summaries . . . . . . . . . . . . . . 79

4.2 Flux Rate Comparison: $\mathrm{PPy}_{b}$ in Varying Feed Solutions . . . . . . . . 81

4.3 Flux Rate Comparison: $\mathrm{PPy}_{b}$ in Solution and Hydrogel . . . . . . . . 84

4.4 ISE Recordings . . . . . . . . . . . . . . . . . 87

$5.1 \mathrm{KCl}_{a q}$ Fickian Diffusivity in Solution and Hydrogel . . . . . . . . . 96

5.2 Flux Rate Comparison: $\mathrm{PPy}_{b}, \mathrm{PPy}_{b} /$ Hydrogel and Biology . . . . . . 98 


\section{Acknowledgements}

I am thankful to my advisors, Pamela Norris and Don Jordan, for the opportunity to work on this exciting project. I could not have found better guides through graduate school; thank you for your patience and endless kindness. Many thanks to my chemistry and electrochemistry gurus, Jerome Ferrance and John Scully: for taking the time to answer my plethora of questions and emails. I'm also thankful for the technical advice, many supplies lent and ideas given by Florent Bocher, Eric Rouya, Kristina Tschulik and the supportive scientific community of my graduate student peers. Thank you to the great friends who helped make dissertation life more bearable outside (and around) the lab: Christina Pappas, Andrea Fiummefreddo, Justin Smoyer, Brian Meffle, Sara Gould, Kimberly Mitchell, and many more. A special thank you to my dear friends far away who always encouraged me to keep going, particularly Maciek Zarzycki and Ariane Lauzon. To my grand-parents Sally Martin and Timothy Wash Haden Sr.; who didn't make it to see me graduate, but were the light that inspired my pursuit of higher education. And to my grand-parents Simone and Jean-Claude Viau, who continue to inspire me every day by their passion for life and kindness. A very special thank you to my sweet and loving parents, Louise Viau and Timothy Wash Haden Jr., who have always believed in me, and who gave me the courage to do anything I set my heart to. And last but not least, to my wonderful husband, Keith William Moored III, to whom I owe more than words can express for his patience and support, for always being there, and for being the best husband I could ever ask for. 


\section{Chapter 1}

\section{Introduction}

The heart is a tremendously efficient biological pump, which beats over 2.5 billion times in an average lifetime [1]. It is composed of billions of cells, called cardiac myocytes, that relax and contract in unison to send blood throughout the body [2]. These cells are considered "excitable" as they require a threshold level of activation to be recruited, and possess refractory periods during which they may not be excited again for a short period of time. These resting phases ensure that the heart beats rhythmically and in a coordinated manner, while preventing contractile waves from traveling back upstream. As a collective, cardiac myocytes are regarded as an "excitable medium" capable of supporting the nonlinear electrical wave propagation that makes the heart beat. Many other examples of excitable media exist in nature, in-

cluding wild fires [3], certain living tissues (e.g. the heart [4], esophagus [5], intestine [6] and nerves [7]), chemical reactions [8, 9], and more [10]. However, a man-made artificial excitable medium has never before been created. The purpose of this research initiative, therefore, was to develop a conceptual design for such a synthetic excitable cell (Figure 1.1), and to demonstrate the feasibility of one of its most critical elements: the artificial cell membrane.

A conceptual model for a synthetic cardiac myocyte (known here as a gel-cell) was therefore developed, with activation phases described. These gel-cells are intended to 


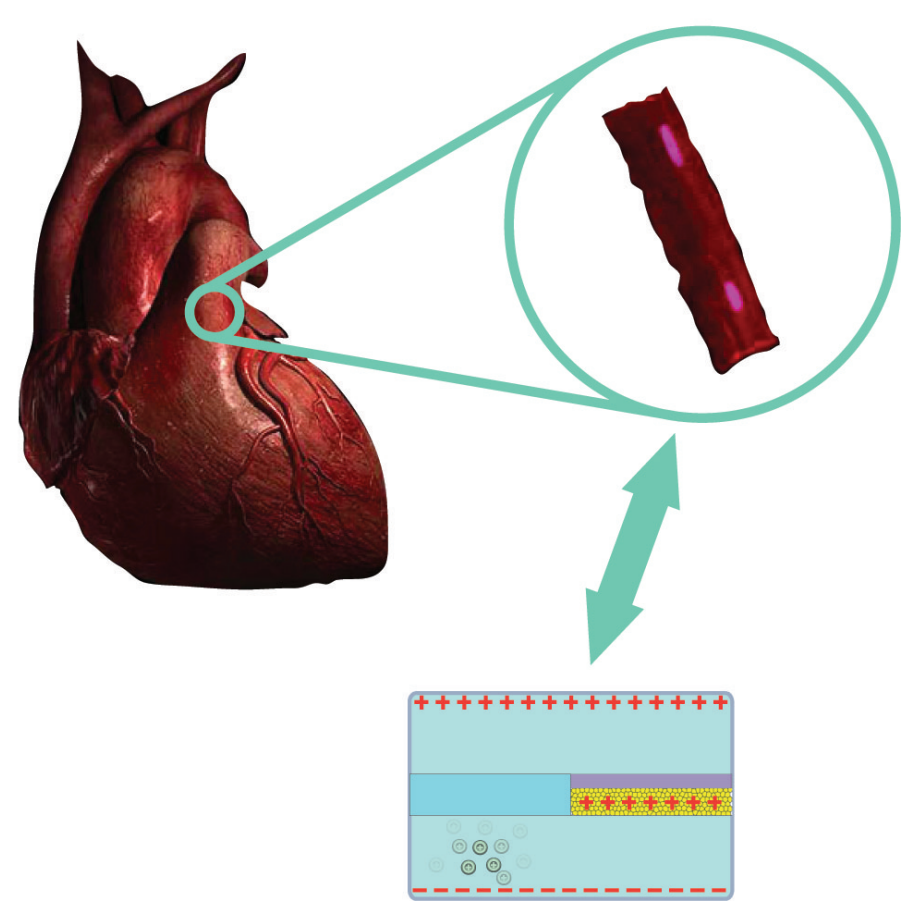

Figure 1.1: The heart is made almost entirely of cardiac myocytes, which are the contractile cells which work in unison to make the heart beat. An actual cardiac myocyte is shown in the magnification in the upper right portion of the image. This research lays the foundation for the development of a biomimetic cell based on such cardiac cells, capable of reproducing its main qualities of excitability and refractoriness.

possess the most basic characteristics of excitability: 1) a resting phase, 2) the ability to sense a threshold level of excitation, 3) an "excited state" where the cell performs its intended activities, 4) a refractory period where the cell is at rest and cannot be re-excited, and finally 5) recovery to the resting phase where the cell is ready to be re-excited [11]. Eventually, coupling these individual gel-cells into a continuous three-dimensional array would enable the propagation of a nonlinear activation wave with the characteristics of a true excitable medium.*

To this end, this research project aimed to answer the question: can the basic quality of excitability found in cardiac cell membranes be reproduced by precisely com-

*The nonlinear activation wave of the excitable medium possesses the following characteristics: 1) a definite threshold for activation below which no signal will propagate, 2) once activated, a pulse of specific shape and speed is propagated without damping, 3) wave fronts mutually extinguish when they encounter one another, 4) pulses vanish without a trace when they encounter a barrier [12], 5) pulses follow a dispersion relation, meaning that the wave speed is a function of how much time is elapsed between activation waves, and 6) the wave speed is a function of the wave front curvature, where a curved wave travels at a noticeably different speed than a plane-wave [13]. 
bined polymer materials? A fully functional and successful prototype of the artificial cell membrane was therefore made using an electroactive polymer embedded in a potassium chloride-rich hydrogel environment. Similar to voltage-gated ion channels (VGICs) in cardiac myocyte membranes, the artificial membrane prototype demonstrated the ability to control transverse ion flux in response to applied potentials.

An electroactive polymer material capable of gating ions in response to the application of a voltage was therefore investigated as a synthetic equivalent for VGICs found in cardiac myocyte membranes. Such biomimetic conducting polymers include polyaniline, polythiophene and polypyrrole [14]. Of these available options, polyaniline's ion gating properties are known to be $\mathrm{pH}$ sensitive [15], which was not a desirable quality for the gel-cell. Polythiophene on the other hand, is most widely used for its variable optical properties [16]. Finally, polypyrrole was chosen for its easy synthesis, the ability to select the dominant ion species for transport [17], and an extensive body of research regarding its ion gating ability [18]. Furthermore, to replicate the ion rich intra- and extracellular spaces found in cardiac myocytes, a semi-solid polymer matrix loaded with liquid electrolytes was sought to construct the main body of the gel-cell. Two main options exist which provide a semi-solid material while retaining liquid ion diffusive behavior within the pores of the material: ionic liquid polymer gels (ILPGs) and hydrogel. ILPG is composed of a molten salt liquid embedded within a polymer matrix, whereas hydrogels are a hydrated polymer matrix which can absorb electrolyte solutions. Although ILPGs were initially chosen for use in the gel-cell, the molten salts were incompatible with the potassium detection technologies used (see Appendix A). Hydrogels, however, were shown to easily uptake large concentrations of electrolytes and posed no interference to a range of ion detection methods used. Hydrogel materials were ultimately selected for these reasons, in addition to other qualities such as their biocompatibility, controllable electrolyte diffusivity, and ease of preparation.

This biomimetic, electroactive polymer membrane represents a critical step to- 
wards the development of the whole gel-cell. The scope of this work included the characterization of both polypyrrole and hydrogel materials for properties relevant to their incorporation into the gel-cell, using such methods as cyclic voltammetry, scanning electron microscopy, equilibrium water content and electrochemical impedance spectroscopy, among others. For example, scanning electron microscopy images of polypyrrole were used to determine their membrane thickness, which were then compared to a theoretical model predicting membrane thickness based on deposition conditions. The model was subsequently improved based on findings from this work, to make it a highly reliable membrane thickness prediction tool. Ion transport studies were also conducted on the polypyrrole gating system in both solution and hydrogel, which provided valuable information on the optimal membrane deposition and activation conditions for peak flux rates. These studies demonstrated the feasibility of the artificial cell membrane based on these materials, and were used to compare its performance to biology. This work also calibrated an ion selective electrode, which was employed in the gel-cell as an ion sensing system for cell activation. These data are presented in detail in Chapter 4, followed by a discussion on their implications as compared to the literature in Chapter 5.

Potential applications for the technology presented here range from the study of cardiac wave propagation to the development of a new class of synthetic muscles. Indeed, connecting the gel-cells into an array would provide a physical model to simulate cardiac wave propagation and better understand the underlying mechanisms for dysfunction, such as with atrial and ventricular fibrillation. In addition, harnessing the known contractile and biocompatible qualities of the materials used in the gelcell could produce a new class of artificial muscle with applications in the field of prosthetics. It would be possible, for example, to capture the biological signaling cues from surrounding muscle cells of an implant, and couple these to an activation signal for the gel-cell array, thereby seamlessly linking biology with the artificial muscle. It is also proposed that the excitable contractile cells could be used as an 
array formed into a tube, creating a bio-inspired peristaltic-type pump where the tube walls themselves propel the fluid forward in waves, similar to the heart. These are only a few examples of potential applications for these gel-cells.

The details for this work will be discussed in the following order. Chapter 2 reviews the relevant background literature, covering the workings of cardiac myocytes and the artificial cell conceptual design. This chapter will also give further details on the polymers employed in this design, and current applications in the literature. Chapter 3 describes in detail the preparation of both the hydrogel and polypyrrole materials, as well as presents the methods for flux rate determination through the membrane. The following section, Chapter 4, will cover the results obtained from the experiments performed on the artificial cell membrane prototype. A discussion is provided in Chapter 5, where the results obtained are considered in detail. A conclusion of this work is found in Chapter 6, where future considerations and overall direction for this project are considered. 


\section{Chapter 2}

\section{Background}

\subsection{Cardiac Myocytes and the Heart}

Cardiac cells, namely cardiac myocytes (see Figure 2.1), are the involuntary muscle cells of the heart, enabling it to contract and circulate blood throughout the body. They are the most recognizable biological example of an excitable unit capable of propagating mechanical and electrical signals when arranged in a continuum [19, 20], and are the biological inspiration for the artificial cells proposed in this research. These cells are on average $90 \mu \mathrm{m}$ in length by $20 \mu \mathrm{m}$ in diameter [21], and are tightly packed one against the other to make cardiac tissue. 


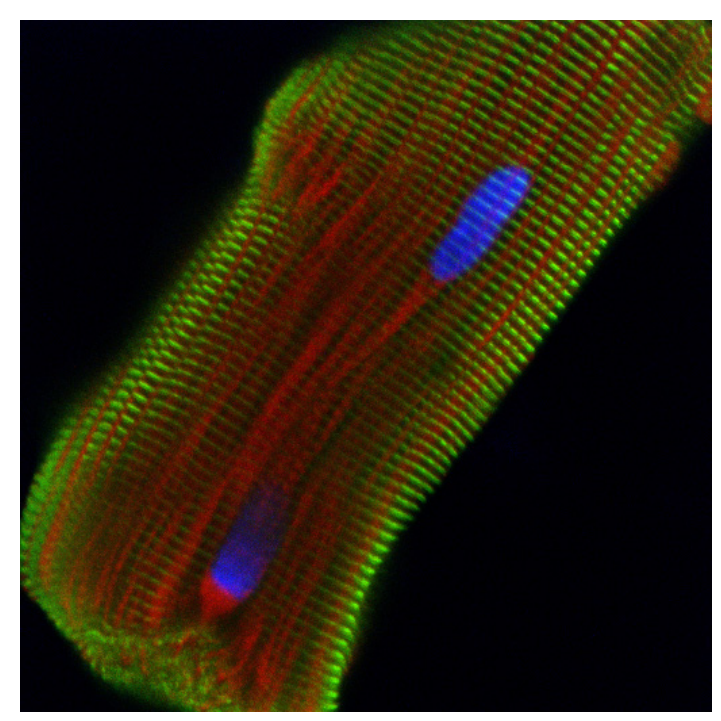

Figure 2.1: Confocal image of a single human cardiac myocyte. Labeled for nuclear DNA in blue, actin filaments in green, and mitochondrial DNA in red. The actin filaments (green) are arranged in a highly defined linear pattern throughout the cell, which is critical as they are part of the contractile mechanism found in cardiac myocytes. Image by Joe Goodhouse from the Confocal Core Lab at Princeton University, reproduced here with his permission.

In the healthy heart, a signal is received from the brain to initiate rhythmic pacing in a subgroup of cardiac cells called the cardiac pacemakers. They have been termed this way because even in the absence of regulatory signals coming from the brain, these cells are able to maintain continuous, cadenced activation of the heart [22]. Each heartbeat starts this way: the pacemaker cells release a biochemical impulse as a signal of their activation, which is passed on through gap junctions to neighboring cells [23]. The membrane potential of these neighboring cells is transiently affected by the incoming ions, causing them to be activated in turn via recruitment of voltage-gated ion channels (VGICs) located in the myocyte membrane. However, this activation is only possible if these cells had sufficiently recovered from their previous activation and are no longer in a 'refractory' state. The refractory period is defined as a time (on the order of a few hundred milliseconds) during which the cell is unable to respond to a second stimulus [11]. Effectively, cellular refractoriness ensures that an activation wave is unable to propagate back on itself, and helps maintain orderly wave propagation down the length of the heart. Because the effective resistance 
for ions is very low in cardiac tissue between cells [24], this activation transfer occurs almost instantaneously, making it seem as though the entire heart beats smoothly, all at once.

More specifically, when charged particles (gap junctional signaling molecules) travel to neighboring cells, they disrupt the carefully and precisely maintained charge gradient which exists across the receiving cell's membrane. Cardiac myocytes sense and respond to this minute depolarization by rapidly activating VGICs located in the cell membrane (Figure 2.2), hence making them "voltage gated". Rapid ion migration across the cell membrane in this fashion leads to a refractory period immediately following excitation, as ions are simply not available to reinitiate cellular pathways immediately after firing.

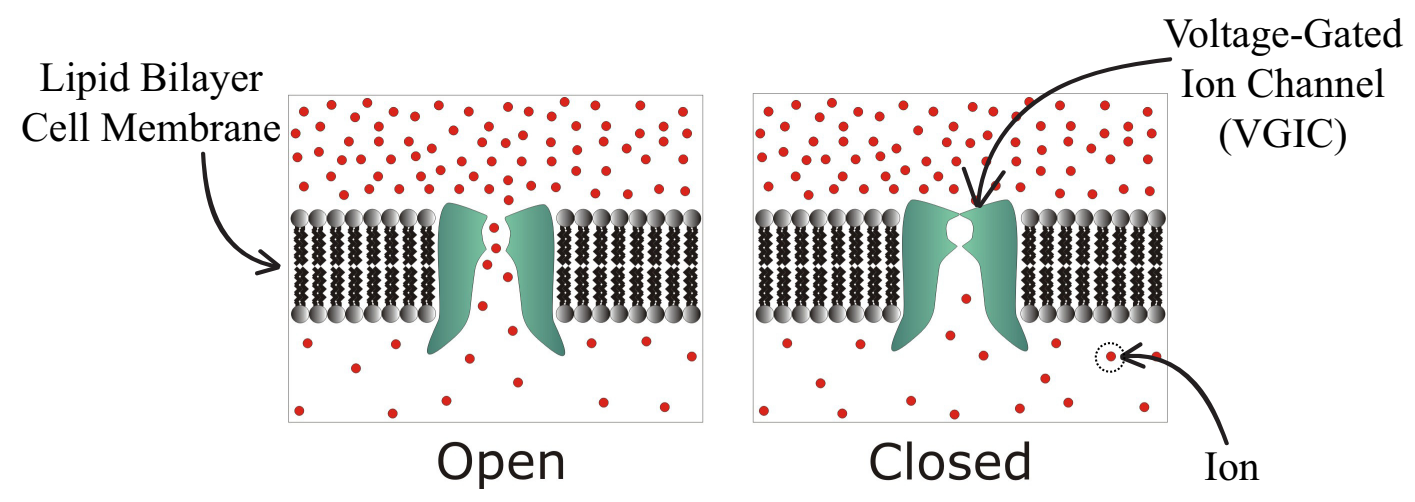

FIgURE 2.2: A schematic representation of the voltage-gated ion channel (VGIC) found in biological cells, analogous to the PPy membrane. Upon activation, VGICs allow high concentration extracellular ions to rush into the cell (left), activating intracellular pathways. Image source: [25].

This depolarization cycle is complex, and involves several ions entering and exiting the cell sequentially (see Figure 2.3); however, the influx of calcium ions in particular is noteworthy as it is responsible for the activation of intracellular signaling pathways leading to cellular contraction. These series of events, culminating in cellular contraction, are collectively known as the "excited state" of cardiac myocytes. These cells are therefore considered "excitable", as an activated cell will keep recruiting neighboring cells to produce a non-linear activation wave that propagates down the length of the heart. 


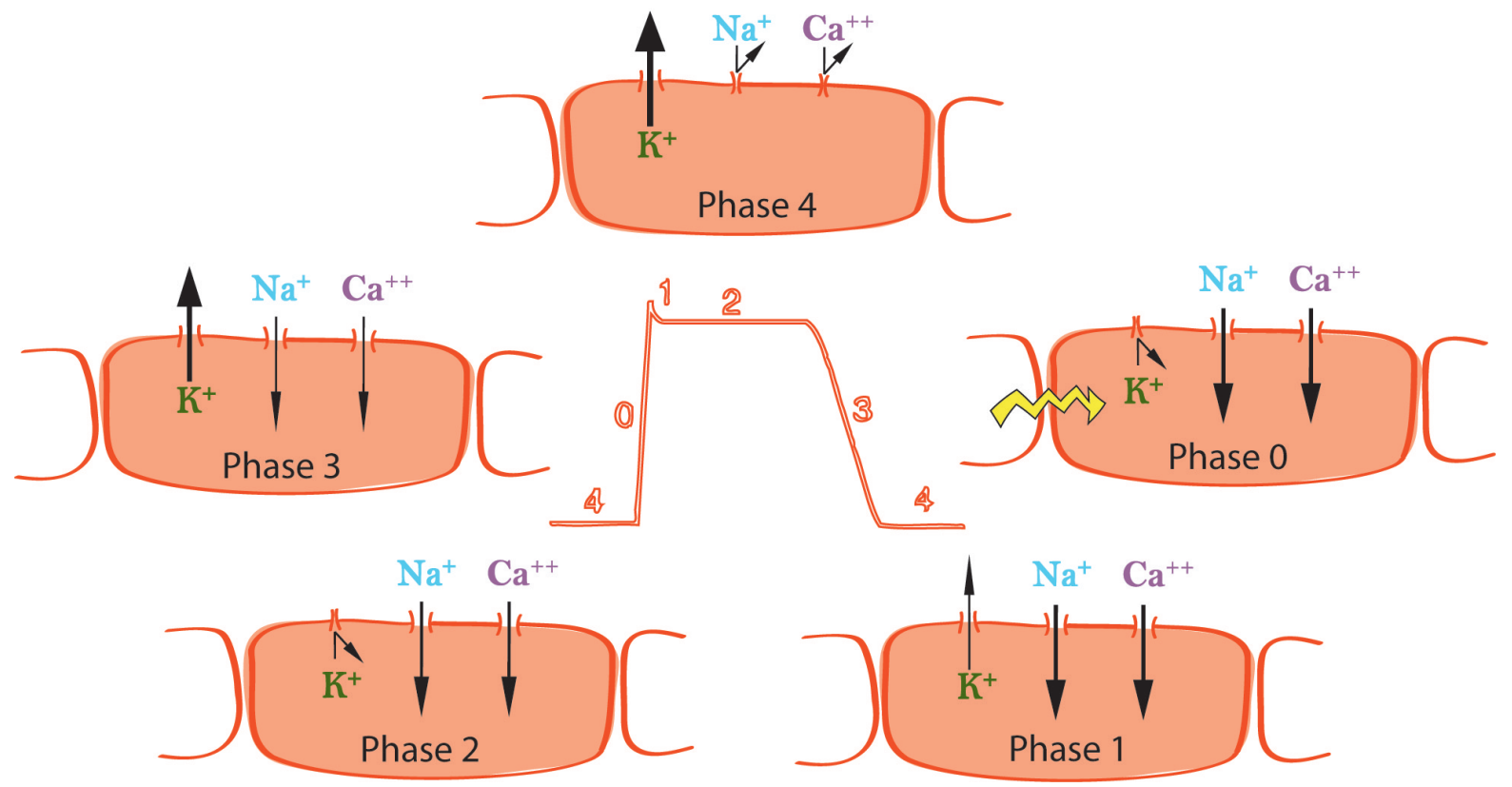

Figure 2.3: Simplified graphical representation of the cardiac cell cycle. Phases 0 through 4 represent the ionic fluxes involved in the cell cycle of an action potential, starting with cell activation in Phase 0 , and ending with relaxation in Phase 4 . They are represented in the center of this image, as the numbers on the action potential curve depicted, showing membrane potential versus time. The cell in the resting state (Phase 4) has a highly negative (around $-90 \mathrm{mV}$ ) membrane potential, due to potassium ions being actively pumped out of the cell [26]. Phase 0 represents the threshold activation of a cardiac cell after its neighbor becomes activated, sending charged signaling molecules via gap junctions. These incoming charges disrupt the cell's membrane potential, and this is sensed by voltage-gated ion channels (VGICs) in the cell membrane. Potassium ion VGICs close, and sodium and calcium channels simultaneously open [27], causing a large change in the membrane potential sending it to around $+30 \mathrm{mV}$. Simultaneously, calcium ions induce a biochemical pathway known as Calcium-Induced-Calcium-Release (CICR), which increases the intracellular calcium concentration nearly a thousand-fold [28]. Calcium flooding the intracellular space then induces contraction by allowing actin to bind and form cross-bridge attachments with myosin, which are the fundamental proteins involved in cellular contraction. Phase 1 is then characterized by a rapid potassium outpouring, which briefly repolarizes the membrane. However, this is short-lived, and the membrane potential plateaus for a few milliseconds in Phase 2, which corresponds to the refractory period of the cell, during which new recruitment could not result in another activation of the cell. In Phase 3 , potassium ion flux is restarted and sodium and calcium channel conductances decrease as their channels are deactivated. The cell is then returned to its full resting state, in Phase 4, ready to be activated again.

The ability to segregate ions on either side of a cell membrane is therefore a key element to the sustenance of life. As shown, nature allows for cellular activation in cardiac myocytes as needed by opening membrane VGICs, which act as the cell's floodgates, responding to changes in the transmembrane potential. Analogous to VGICs, electroactive polypyrrole (PPy) membranes, which were used in this project to mimic the biological membrane, also allow for the segregation and accumulation of 
ions to one side of the membrane, and upon voltage activation, the rushing of these ions across it $[29,30]$. These biomimetic polymer membranes will be central to the research presented, and will be discussed in more detail below.

In the diseased heart, signal transmission between cardiac myocytes is disrupted [31-33], and refractory periods are no longer clearly defined. This causes cells to be activated upstream again before the end of their refractory period, thus creating highly irregular wave reentry patterns throughout the heart (Figure 2.4, A and B). These wave propagation patterns cannot sustain a normal cardiac rhythm, and cause the heart to quiver instead of beat, which is known as cardiac fibrillation [34].
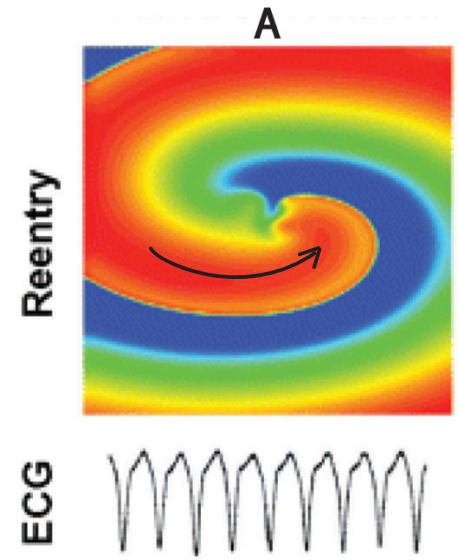
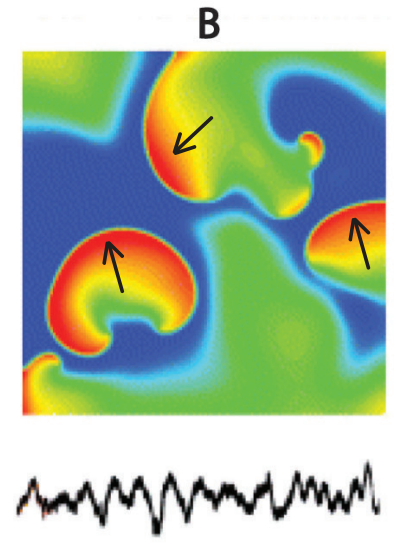
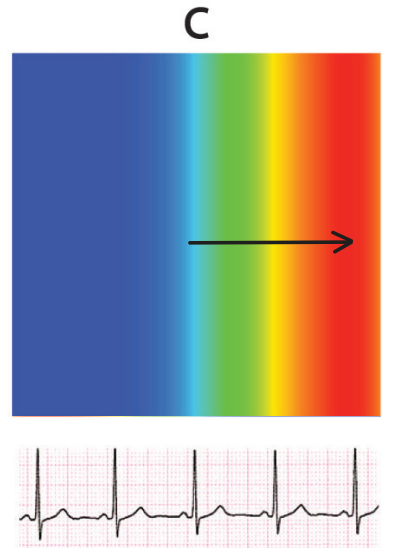

FIGURE 2.4: Examples of simulated cardiac wave propagation and their respective electrocardiogram (ECG) traces. The color gradient represents cellular recruitment, with red having most recently been activated, and blue corresponds to tissue at rest. Arrows indicate direction of wave travel. A) A reentrant wave begins, disrupting normal sinus rhythm. B) Instability of the reentrant wave instigates further wave disturbance, leading to the inability of the heart to contract normally. C) Example of a normal sinus rhythm propagating from left to right across the simulated tissue, and its associated ECG. Image adapted from Qu et al. with permission from Wiley. [35]

There are two major types of cardiac fibrillation: atrial and ventricular, which refer to the location of their occurrence in the heart. While the atria receive both oxygenated and deoxygenated blood from the rest of the body, the ventricles are responsible for pumping this blood back into the body. For this reason, ventricular fibrillation (VF) prolonging for more than a few minutes results in cardiac arrest and 
sudden cardiac death (SCD) [36], as the heart is no longer able to circulate blood throughout the body. Treatment for VF is therefore needed immediately after its onset, and typically includes defibrillation or cardioversion, which are highly successful rescue therapies. Atrial fibrillation $(\mathrm{AF})$, on the other hand, is not instantly fatal but can ultimately result in significant morbidity and mortality. Although treatments used to restore sinus rhythm in VF patients can also be used in the case of AF, such aggressive treatments as defibrillation and cardioversion would be best replaced by more sophisticated preventative and eradication therapies. AF is characterized by tachycardia (abnormally fast heartbeats), resulting in a fatigued and eventually enlarged heart. This can in turn lead to the formation of blood clots, the occurrence of strokes, and even heart failure [37]. AF is the most common type of arrhythmia found in developed countries, affecting over 2.2 million people in the United States in 2009 [38]. These two types of arrhythmias (AF and VF) remain major health problems today, claiming the lives of over 461,000 Americans in the same year [38], despite decades of research aimed at understanding their underlying initiating mechanisms.

Treatments for AF currently include anti-arrhythmic drugs (AAD) and/or radiofrequency ablation (RFA). Radiofrequency ablation is a technique in which catheters are sent into the atria of originating reentry behavior, and high frequency waves are used to burn small incisions in the tissue at random locations, in an attempt at forcing a sinus rhythm on the heart. As compared to drug therapies, RFA treatments report higher efficacy rates $(71 \%$ versus $52 \%)$ and lower complication rates $(5 \%$ versus $30 \%$ ) [39], but are still not based on a precise understanding of reentrant wave initiation, which could improve treatment efficacy rates significantly. One of the longterm scientific ambitions of this research, therefore, is to develop a physical model of cardiac tissue to simulate and study cardiac fibrillation initiation and sustenance, and to assist in the improvement of treatment options.

Currently, a better understanding of the wave propagation disruption in AF is afforded by biological experiments and computer simulations. In the biological ex- 
periments, AF is induced in animal models, which are either chronically instrumented with an array of electrodes placed in the heart to detect arrhythmias [40-43], or sacrificed to observe protein expression $[44,45]$ and wave patterns using a voltage sensitive dye [46-48]. While biological experiments are necessary for their insight into cellular pathway activations occurring in situ, they are also constrained by their need for animal models and the limited spatiotemporal information these relay, as basket catheters placed in the atria carry restricted numbers of electrodes [49-52], and voltage-sensitive dyes give only two-dimensional wave propagation information at the surface $[53,54]$. Even studies recording the transillumination of voltage-sensitive dyes encounter difficulties with signal-to-noise ratios and cannot accurately evaluate wave propagation in 3-D [46]. To overcome this limitation and uncover the critical 3-D characteristics underlying fibrillation, computer simulations have been developed which are capable of modeling the wave propagation occurring in the heart $[55,56]$. Although highly accurate and physiologically relevant as they are based on the models of ionic transport across the cells membrane, the Nernst equation, and physiological data; they too are limited by their ability to only model a restricted number of elements due to their computational complexity [57]. Most recently, computer simulations have managed to model up to around 100,000 nodes for the heart [58], but this is still a far reach from the $>200$ million elements needed to properly treat activity at a cellular scale [59].

The breakdown of orderly cardiac wave propagation to a turbulent or fibrillatory state is enormously complex, and the treatment of cardiac fibrillation is very difficult. Data on the efficacy of currently available treatments reflect a persisting lack of fundamental understanding into the mechanisms that initiate and sustain the disrupted cardiac wave propagation on a biochemical and structural level. In fact, an expert panel from the National Heart, Lung, and Blood Institute convened in 2008 suggested that, among others, there was a great need for improving non-invasive technologies which could help better understand the influence of heart structure on 
the incidence of AF [37]. Indeed, the development of a 3-D, structurally accurate, biomimetic artificial heart has the potential to provide a better understanding of 1 ) the loci of AF formation, 2) where to best focus RFA therapy, and 3) the effects of cardiac geometries on wave propagation and reentry sustainability.

Molding a coupled array of micron-scaled gel-cells could produce a physiologically accurate model of the shape, layout, and thickness of the human heart. Electrical activation signals produced by each artificial cell could then be read by a computer, effectively mapping a 3-D representation of the wave propagation as it occurs in realtime through the geometry of a heart. This information could help better understand the effects of geometry on cardiac wave propagation, which are believed to be of great importance in the initiation and maintenance of AF [60].

\subsection{A Biomimetic Heart Cell}

In order to achieve the long-term goals outlined above, the aim of this work was to develop the conceptual design of an artificial excitable cell, determine the materials required for its fabrication, and demonstrate its feasibility through a prototype of the most critical portion of the gel-cell: the biomimetic cell membrane. The work outlined here lays the foundation for the eventual production of a complete gel-cell prototype, to be used as a building block in engineered excitable media. 


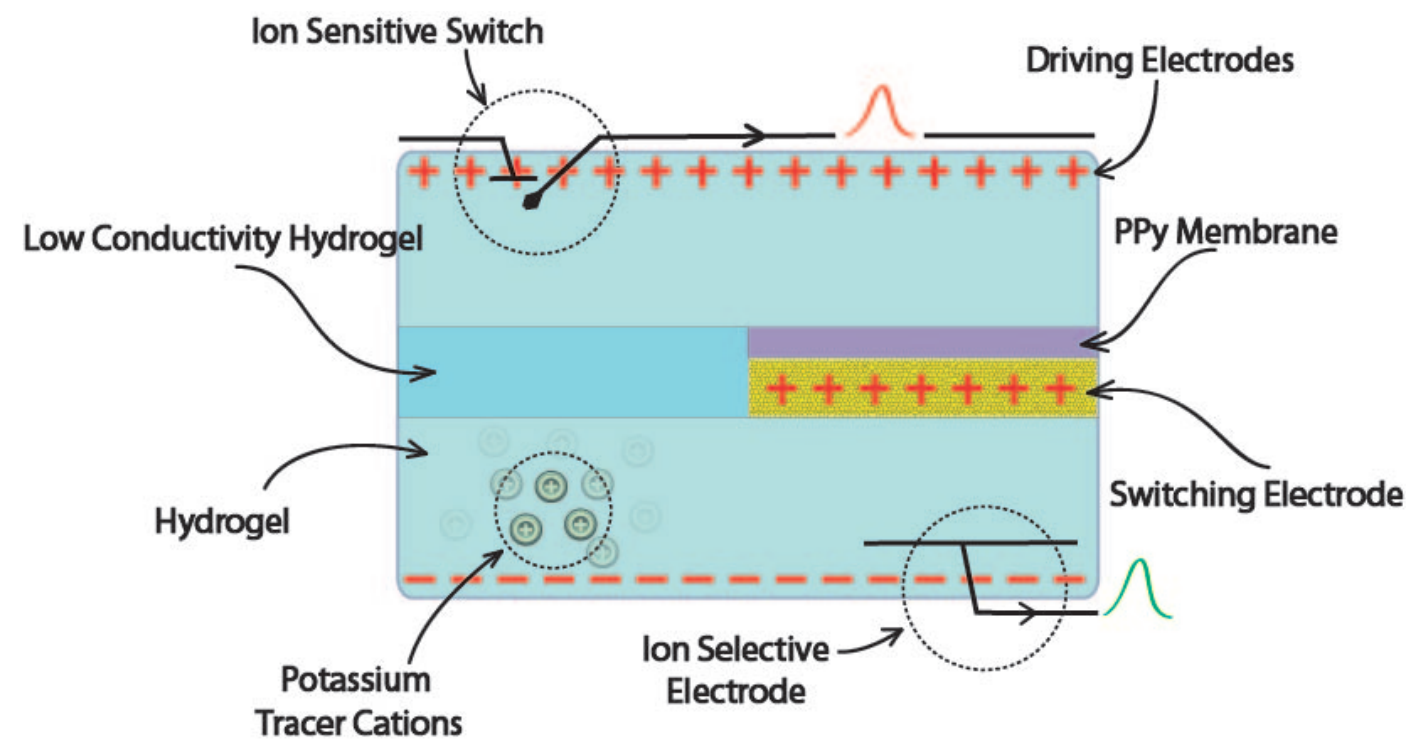

\section{Cell Schematic}

Figure 2.5: Conceptual design of the artificial excitable cell layout. Hydrogel comprises the main body of the cell (light blue hues). A PPy membrane (purple) controls the ionic flux from the upper portion to the lower portion of the cell. Potassium ions are free to move about the cell body, however, they have a harder time moving through the lower conductivity hydrogel (darker blue, middle left), and hence preferably move through the PPy membrane when it is open. An ion sensitive switch and a potassium ion selective electrode are used to ensure excitability and refractoriness of the cell by ensuring a lapse in time before re-excitation, and signaling when the cell has been activated.

Electro-active polymers (EAPs) were selected in order to control and direct ion transport, and to measure localized ion concentrations, thus reproducing the main events of the cardiac myocyte activation cycle. Hydrogel was chosen to make up the bulk of the upper and lower chambers of the gel-cell (Figure 2.5). Mimicking the ion-rich environments of biological cells, ions are free to move about in hydrogel material, making it a suitable foundation for the gel-cell [61]. For simplicity, instead of having a variety of ions permeating around the cell as found in biology, the gel-cell is simply filled with a potassium tracer ion.* As these ions cycle through the gel-cell, they are transported in a unidirectional, clock-wise cycle around the cell, driven by voltage gradient fields produced by electrodes placed at the top and bottom of the

*Potassium was chosen since it has the smallest hydrated shell and the fastest rate of flux through the gating membrane [62]. However, sodium ions could also be used instead, since they have a flux rate very similar to that of potassium: $\mathrm{K}_{q}=5.74 \times 10^{-9} \mathrm{~mol} \cdot \mathrm{s}^{-1} \cdot \mathrm{cm}^{-2}$ and $\mathrm{Na}_{q}=5.63 \times 10^{-9}$ $\mathrm{mol} \cdot \mathrm{s}^{-1} \cdot \mathrm{cm}^{-2}$. 
cell design.

An ion gating membrane made of polypyrrole (PPy) separates the upper and lower chambers of the gel-cell, mimicking the biological cell membrane described above. Similar to VGICs found in the biological cell membrane, a PPy membrane remains impassible to ions until activated, when it funnels them into the lower chamber of the gel-cell. Before opening the PPy ion gate, however, the gel-cell must first determine whether it is in the correct phase to respond, i.e. not in a refractory period. It does so by probing the local ion concentration in the upper chamber using an ion-sensitive switch [63].

Once ions migrate to the lower portion of the cell, they trigger an ion selective electrode (ISE) probe placed there to produce a voltage, which is used to propagate the signal of activation to neighboring cells. After this step, ions are driven back to the upper chamber via electrodes to restore its resting state; but this time they travel through the low-conductivity hydrogel in a slow diffusive process, as the PPy gate has been closed. When a sufficient concentration of ions has reached the upper chamber of the cell again, it is then available for re-activation, as determined by the ISE probe and switch located there.

\subsubsection{Operating Sequence for an Excitable Gel-cell}

The gel-cell is designed to cycle through five main phases, undergoing a process similar to the cardiac myocyte activation cycle. A brief description of the events occurring in each phase are found below, along with a schematic representation in Figure 2.6.

Phase 4 The gel-cell is in the resting phase and ready to be fired. The upper driving electrode is negatively charged to attract the tracer cations. The PPy membrane is closed and impassable to the tracer ion.

Phase 0 The gel-cell receives a signal to fire (green pulse) from a neighboring cell, 


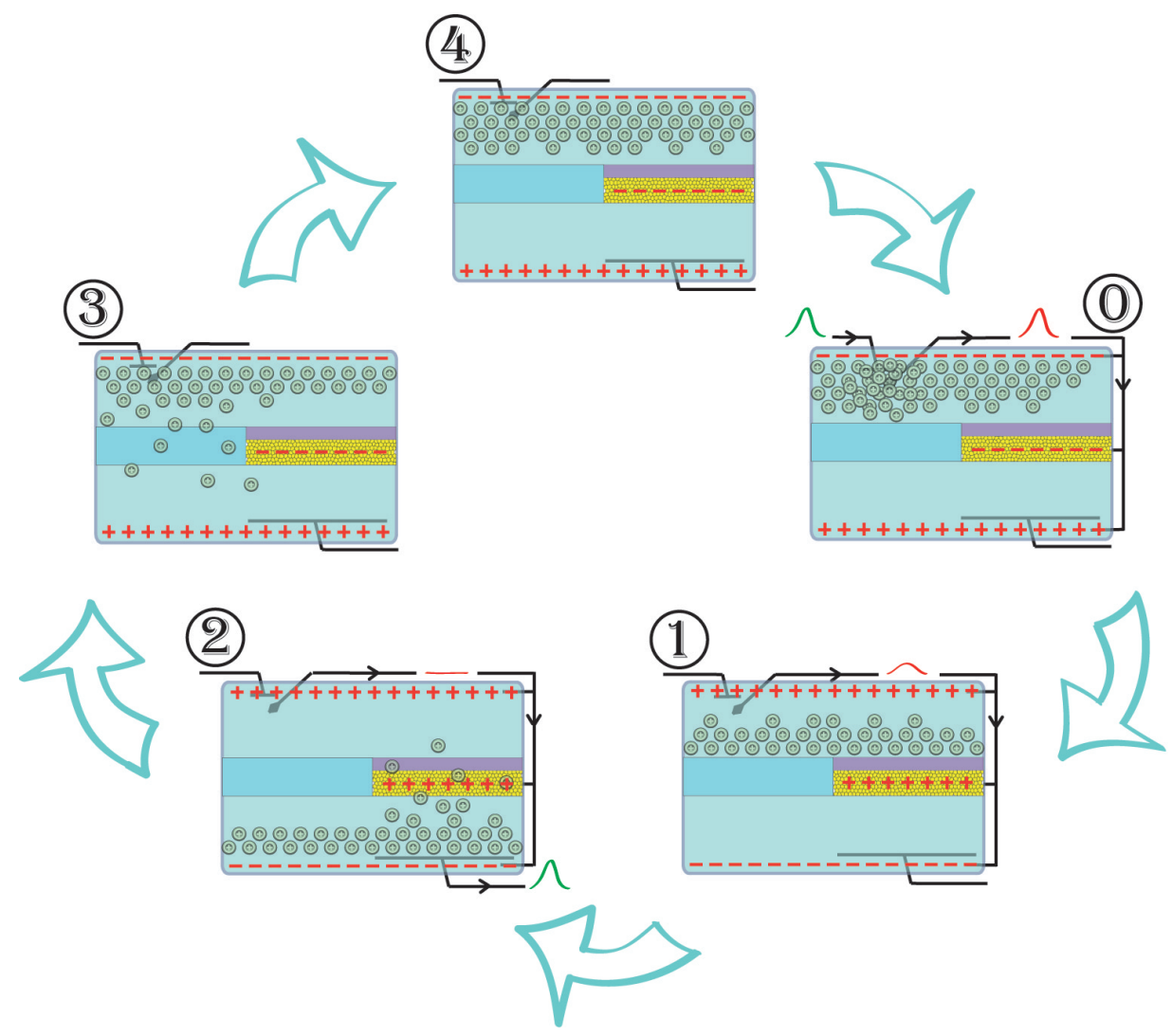

Figure 2.6: A sequence diagram illustrating the five phases in the artificial excitable cell cycle. Potassium tracer ions go through a clockwise rotation around the gel-cell as they are directed by electric fields down through the PPy membrane upon cell activation, and back up through the low-conductivity ILPG upon their return to the resting state.

analogous to gap junctional conduction in myocytes. This signal charges a negative electrode located in the ion-sensitive switch, increasing the local tracer ion concentration (if available) above threshold. An ion-selective sensing electrode relays the local concentration of ions by opening transistor gates in the switch, thus allowing the activation signal to continue to the rest of the cell (red pulse).

Phase 1 A polarity reversal at the driving electrodes causes the tracer ions to migrate to the barrier of the low-conductivity hydrogel and PPy membrane, but cannot yet pass through the closed PPy membrane. A positive voltage is concurrently being supplied to oxidize and open the PPy gating membrane.

Phase 2 The PPy membrane rapidly becomes permeable and tracer ions pass into the lower chamber. Ionic flux into the lower chamber occurs preferentially 
through the PPy membrane as it offers the path of least resistance to ionic movement. The ion selective electrode (ISE) sensor in the lower chamber measures an increasing ion concentration and outputs an increasing voltage analogous to the action potential found in cardiac myocytes. Furthermore, as tracer ions leave the upper chamber, the ion concentration there drops and the ion-sensitive switch reverses to its initial state, causing the PPy membrane to become impermeable to ions again. At this point the gel-cell is in the refractory stage and cannot be fired again.

Phase 3 The driving electrodes have now returned to their baseline polarities, and the PPy membrane is impermeable to ions again. The tracer ions are forced to diffuse back up through the low conductivity hydrogel. The amount of time it takes for ions to pass through this area dictates the refractory period duration for the gel-cell.

\subsubsection{Biomimetic Cell Components}

The main components of the artificial cell are: 1) the hydrogel medium, which is the polymer equivalent to the biological intra- and extra-cellular space, 2) the polypyrrole (PPy) gating membrane, analogous to a cell membrane with voltagegated ion channels (VGICs), 3) the potassium sensitive electrode, and 4) an ion sensitive switch. The scope of the project presented here covers the production and testing of the first three gel-cell components, as they are most critical to the overall conceptual design's feasibility. Future work will involve combining all four major elements to produce the fully assembled artificial excitable cell prototype. A review of each component and the details of their fabrication methods are discussed below. The results obtained from testing the first three components will be further discussed Chapter 4. 


\subsubsection{Polypyrrole}

Polypyrrole is a thin electroactive polymer membrane, produced by electrochemical polymerization from pyrrole. It is a very versatile polymer due to its responsiveness to applied voltages and has been used, among others, as an ion gate, potassium selective electrode, actuator, transistor, supercapacitor and in batteries [64-69]. Of particular interest in the context of this research, is its ability to act as a gating membrane capable of controlled ion permeability, opening and closing in response to an applied voltage as it switches between oxidation states, a behavior analogous to VGICs.

Polypyrrole (PPy) is a polyheterocycline organic polymer made from pyrrole (Figure 2.7), which was first reported synthesized in the early 1960's [70-72]. By the 1980's, PPy was gaining attention as a novel metallic polymer, due to its uncharacteristically high electrical conductivity, ranging from 10-100 S / cm at room temperature $[73,74]$. These high electrical conductivities are due to the overlapping and partially filled molecular orbitals of pyrrole, which allow for electron mobility throughout the $\pi$-conjugated polymer lattice $[75,76]$.

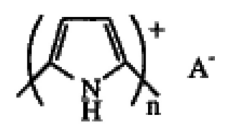

Figure 2.7: Chemical structure of pyrrole, the monomer which forms a polypyrrole chain after deposition.

In 1982, Burgmayer et al. discovered an additional capability for polypyrrole; this time a variable ionic conductivity. Their group noted the polymer's ability to switch between oxidative states in response to low voltages, and how these corresponded to two distinct ionic permeability states, one open and one closed; making it useful as a switchable ion gate [77]. Since then, more in-depth studies have shown that the nature of the counterion placed into the membrane during polymerization controls 
the polarity of the ions allowed to pass through the membrane [78].

Several uses for polypyrrole membranes have further emerged since their discovery. A wide variety of applications harness the myriad of mechanical, optical, chemical and electrical properties displayed by these fascinating polymers. For example, PPy responds to a voltage application by swelling or relaxing, depending on ionic uptake, to preserve electronegativity. This has been harnessed to provide motion, and several studies have demonstrated the actuating capability of PPy membranes [79-83]. They are additionally being investigated for use in solar cells, rechargeable batteries, biosensors, electrochromic devices, anti-static coatings and many more [75, 84-91].

The membrane is formed from the electrochemical polymerization of a pyrrole monomer and a counterion of choice, onto the working electrode of a 3-electrode electrochemical cell (see Chapter 3 for more details). Deposition time dictates membrane thickness, which is on the order of a few microns [92]. These films possess a characteristic cauliflower-like morphology under scanning electron microscopy (SEM, see Figure 2.8). Transport of a variety of ions through these membranes has been demonstrated, including potassium ions [93]. In fact, potassium ions have a very rapid flux rate through PPy membranes due to their small hydrated ion diameter, or effective ionic radius of $3 \stackrel{\circ}{A}[62]$.
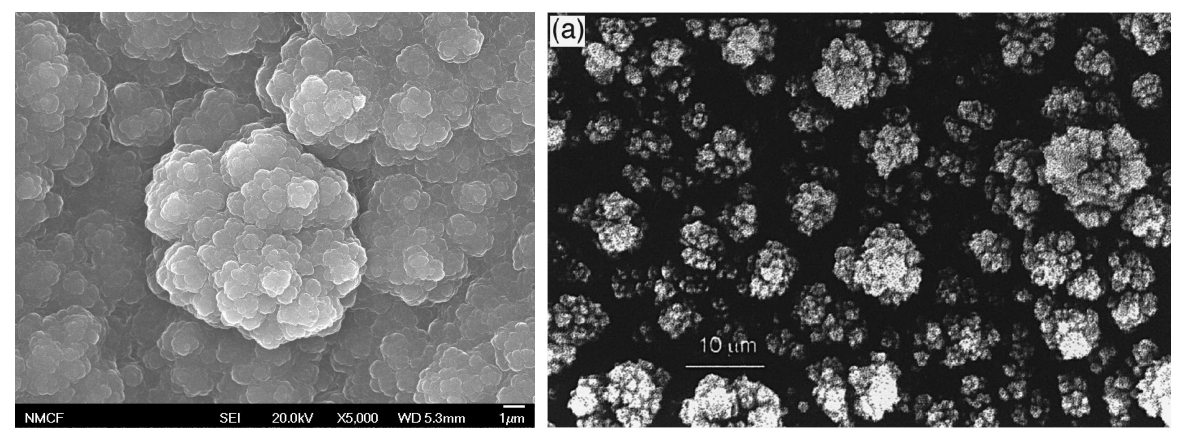

FIgURE 2.8: Scanning electron microscope (SEM) imaging of PPy membranes. Left: Image taken at UVa of a PPy membrane produced in the Soft Materials Lab. Right: SEM image taken by Davey et al., 1999, reproduced with permission from Elsevier. Notice the features at the same length scale are nearly identical in both images.

Polypyrrole's ability to gate ionic conductivity is based on its electrochemical re- 
dox state (Figure 2.9). Upon the application of a positive voltage, the PPy membrane becomes oxidized and in this state is permeable to ions. Conversely, upon the application of a negative voltage, membranes are chemically reduced, and their permeability to ions is decreased about a thousand-fold [29]. This ability to control ionic flux makes PPy membranes analogous to a biological membrane and therefore very useful in the design of the gel-cell.
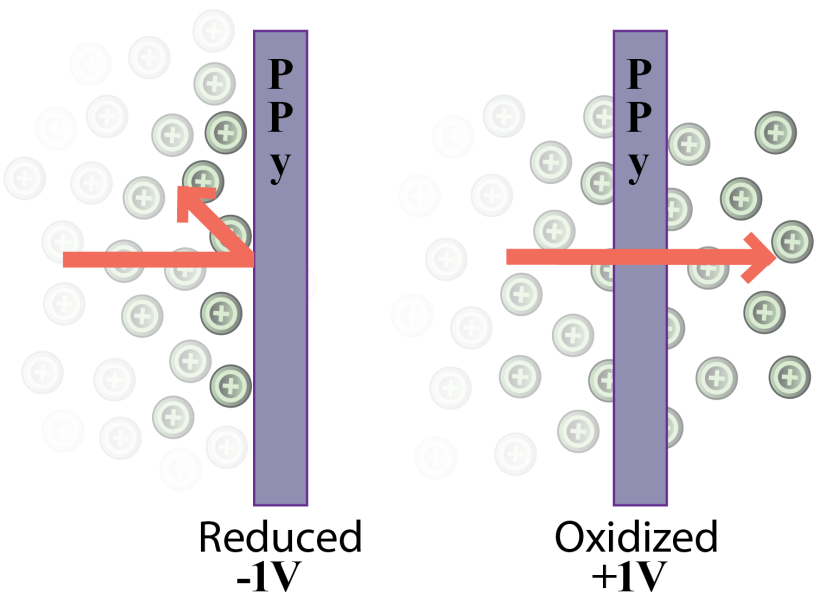

FIgURE 2.9: PPy is an electro-active thin membrane produced through electrochemical deposition. Upon application of a negative voltage to the membrane $(-1 \mathrm{~V})$, the membrane is chemically reduced, and in this state is impermeable to ions (left). When the voltage is reversed $(+1 \mathrm{~V})$, the membrane becomes chemically oxidized and is open to ion passage (right).

\subsubsection{Hydrogels}

The ideal material base for the gel-cell is a semi-solid, aqueous environment in which potassium chloride $(\mathrm{KCl})$ is easily incorporated and traced. The material that best suits these criteria is a water swollen polymer network, known as hydrogel. Hydrogels are made from polymers which would be glass-like if produced in the absence of water. In its presence, however, over $20 \%$ of its weight in water is absorbed by the polymer matrix, producing an elastic gel material instead [94]. The equilibrium water content, EWC (see Eq. 2.1), is a measure of the amount of water absorbed by hydrogels and is a function of the hydrophilicity and network structure of the base polymer [95]. It dictates mechanical properties of the gel, ion permeability, and can be 
correlated to hydrogel pore size, making it the most valuable and telling characteristic of hydrogels [96].

$$
\mathrm{EWC}=\frac{\text { weight of water in the gel }}{\text { total weight of swollen gel }} \times 100 \%
$$

Hydrogels can be made from a broad range of polymers. A few examples include 2-hydroxyethyl methacrylate (HEMA) [97], acrylamide (AAm), N,N'-methylene-bisacrylamide (MBAAAm) [98], N-vinyl pyrrolidone (VP) [99], polyvinyl pyrrolidone (PVP) [100], polyvinyl alcohol (PVA) [101], and methyl methacrylate (MMA) [102]. They have been used in a wide variety of applications: as biomaterials in drug release and soft contact lenses [94, 103], in agriculture [104] and electrophoresis [105]. Hydrogels have also been used in microfluidics, which motivated the development of new methods for the direct photopatterning of these materials on a micrometer-scale [106]. Producing large arrays of micropatterned gel-cells becomes a reality in light of these technological developments.

There are three classifications for hydrogels based on pore size: 1) macroporous hydrogels contain pores larger than $100 \mathrm{~nm}, 2)$ microporous hydrogels with pores between 5 and $100 \mathrm{~nm}$, and 3) nonporous hydrogels which do not contain any true pores [96]. Direct and indirect methods for pore size determination exist. Examples of direct pore size determination include electron microscopy [107], the bubble point method [108], gas adsorption [109], differential scanning calorimetry [110], NMR spectroscopy [111], Fourier transform infrared spectroscopy imaging [112], and more [113]. Indirect measurements involve the use of theoretical models to estimate pore size information, such as the Equilibrium Swelling Theory [113].

Since none of these direct analysis methods were readily accessible to the Soft Materials Lab, the polymer ratio to cross-linking agents were correlated to known porosity data found in the literature. The acylamide hydrogels chosen for this project were based on two recipes produced by Barthus et al. [114], who determined their pore sizes to be an average of $0.52 \mathrm{~nm}$ and $2 \mathrm{~nm}$ by Equilibrium Swelling Theory, 
and correlated to polymer cross-linker ratios. An even smaller pore size hydrogel recipe was prepared for the purposes of this work, with a polymer ratio of 0.065 and estimated pore size of $0.1 \mathrm{~nm}$.

In addition to the above qualities, hydrogels have good demonstrated compatibility with the electroactive polymer polypyrrole (PPy), which has a central role in the artificial cell design. In fact, Barthus et al. showed that PPy could be electrochemically prepared within a hydrogel environment, in the case of a controlled drug release device [114]. Hydrogel's ability to swell and contract depending on environmental conditions makes them interesting for use in drug delivery system [115]. In fact, they can be made to respond to cues such as temperature, $\mathrm{pH}$, light, electric or magnetic fields simply by changing the end group chemistry on the polymer chains [116-120]. Interestingly, this ability to swell and shrink led Kuhn et al. to dub them "chemical muscles" in the early 1950s, in one of the very first descriptions of hydrogels [121].

Work has also been done by Horkay et al. to study the interaction between the hydrogel polymer structure and alkali metal salt solutions (such as aqueous potassium chloride) at physiologically relevant concentrations [122], confirming their ability to be integrated into hydrogels. Horkay's group described the theoretical distribution of ions within a hydrogel network based on the principal of electronegativity, free energy and Bjerrum length. They found that if the distance between ions is much larger than the characteristic (Bjerrum) length, then ions would be distributed uniformly throughout the gel. If the opposite were true, however, ions would be closely bound to the polymer chains, in a process known as counterion condensation. The elasticity of the polymer was not affected by the presence of any of the ions either, meaning that there is likely no association between the polymer chains and ions in solution. This quality maximizes the mobility of ions within hydrogels, making it another desirable attribute of these polymers. 
2.2.2.1 Solute Diffusion Several studies have quantified $\mathrm{KCl}_{a q}$ flux through hydrogels as well $[98,123,124]$, and determined theoretical models for ion transport [125]. Capillary pore diffusion and solution-diffusion models were used to describe solute diffusion through PMMA and other materials used as dialysis membranes [126]. According to these studies, the speed of solute diffusion in hydrogels is dictated not only by chemical interactions between ion-ion, ion-water and ion-polymer; but also by the characteristics of the polymer medium through which it passes including pore size, tortuosity and water content.

In the simple capillary pore diffusion model by Pappenheimer [127], perpendicular pores of equal diameter run the length of a material, and flow through them follows Poiseuille's law. This model is overly simplistic, however, and was modified by Verniory [128] to include frictional resistance in consideration of Stoke's law describing flow of a sphere through a channel. Yet another description of solute behavior in hydrogels, the free volume model of diffusion, correlates water content in polymers to ion mobility [129], which could be helpful, but fails to account for the chemical interactions of the solute with polymer chains.

2.2.2.2 Biocompatibility A long term goal for this project is to eventually interface gel-cells with living tissue. To this end, material biocompatibility must be a consideration. Luckily, many examples already exist of hydrogels interfacing with the human body, including soft contacts [94, 103], burn dressings [130], cell culture [131], artificial cartilage implants $[132,133]$ and drug delivery [134]. It is the high water content of hydrogels which leads to good biocompatibility; as impurities can easily be washed out before use, the soft and pliable nature of the material reduces mechanical stress on living tissues, and provides low interfacial tension [96]. 


\subsubsection{Ion Selective Electrode}

A versatile polymer, polypyrrole has also been used as a solid state ion-selective electrode $[135,136]$. It does so by binding ions in solution to the ion exchange counterion within PPy, which in turn produces a voltage drop with respect to a reference electrode. However, the PPy and ion exchanger combination have a poor detection limit. The addition of ionophores such as valinomycin [137] or a crown ether [138] in polyvinylchloride (PVC) to the surface of PPy has been shown to greatly increase the detection limits for these sensors. In fact, Pandey et al. [138] showed a detection for potassium ions as low as $15 \mu \mathrm{M}$ by the addition of dibenzo-18-crown- 6 in PVC to the PPy membrane (see Figure 2.10).

The long-term stability of PPy potassium sensors was investigated by Momma et al. [139], who showed that the thicker the PPy membrane base, the higher the stability of the ion-selective electrode over time. They also demonstrated that the sensor fared better in higher concentrations of $\mathrm{KCl}_{a q}$ than in lower ones, likely due to the structural stability afforded by the presence of the binding agent. These properties are each useful in the design of the gel-cell, and will be used to detect the local availability of potassium chloride in the various steps of the activation cycle. 


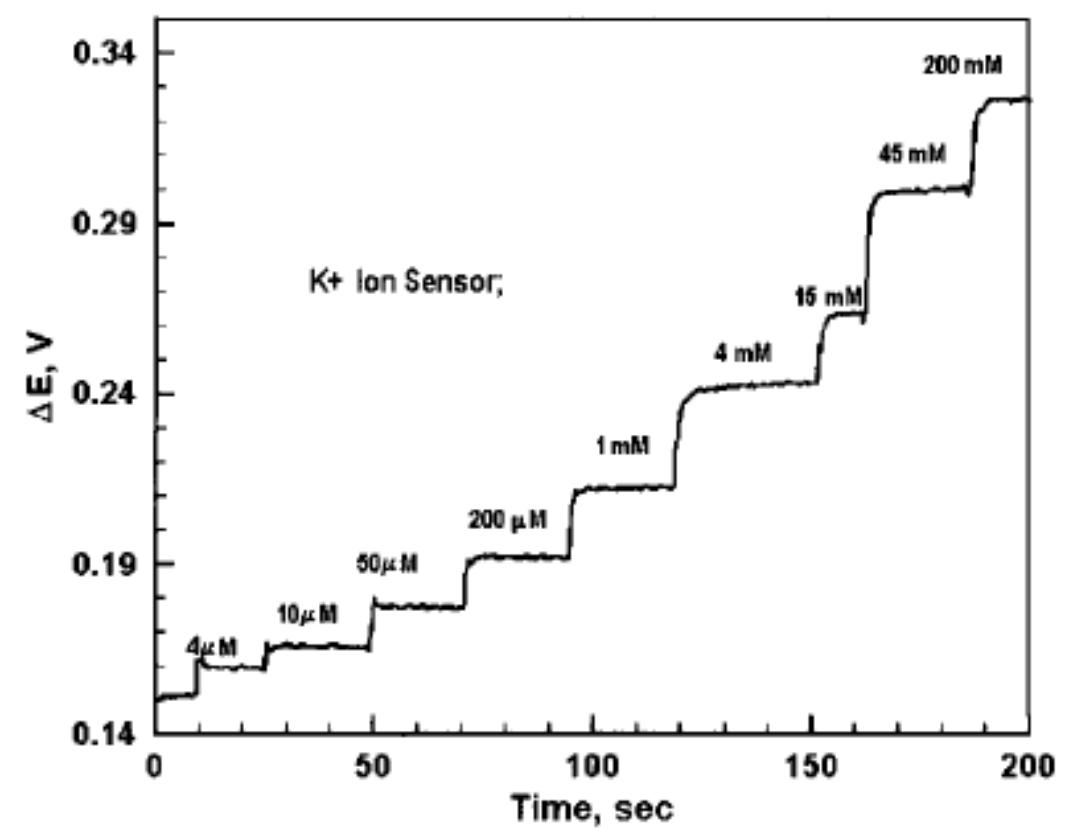

FiguRE 2.10: Voltage drop of PPy doped with dibenzo-18-crown in response to varying concentrations of $\mathrm{KCl}_{a q}$, as seen by Pandey et al. Reprinted from [138], with permission from Wiley.

\subsubsection{Ionic Switch}

The inclusion of a novel ion-sensitive switch will be critical for the development of an excitable gel-cell. The ion-sensitive switch has yet to be fully designed, and is left for future consideration. The concept however will likely contain the ISE described above, and soft polymer transistors for gating logic.

\subsubsection{Potential Applications of the Artificial Cell}

The development of an artificial excitable cell prototype and its testing are the first steps in the larger scope of a research project aimed at producing artificial excitable tissues. There has been a large body of work aimed at producing whole artificial cells, as reviewed in a recent book by Chang [140], however, none have used PPy as an analogous cell membrane in the way this research demonstrates. Eventually, the biomimetic gel-cells developed here will be connected with one another, and produced at the micron scale. Large numbers of these will then be coupled into 3-D arrays, and 
molded into complex shapes. A variety of applications will become possible through the development of such a novel artificial excitable tissue.

For example, if the artificial tissue were constructed into the shape of a physiologically accurate heart, it could be used to model the complex wave propagation and re-entry behavior seen in atrial fibrillation. Physical modeling of cardiac wave propagation and the initiation of arrhythmias would complement current research into the fundamentals of cardiac fibrillation by uncovering the critical 3-D wave propagation characteristics in real-time. This would provide a powerful alternative to computationally intensive simulations which can only capture activity through small sections of the heart at a time. Indeed, producing a physiologically accurate model of the heart could provide a better understanding of 1) the loci of AF formation, 2) where to best focus RFA therapy, and 3) the effects of cardiac geometries on wave propagation and reentry sustainability, thereby improving our fundamental understanding of the influence of heart structure on the incidence of AF.

Gel-cells also have the potential to become a new class of artificial muscle, by harnessing the inherent contractile qualities of the materials from which they are made. Indeed, both polymer materials used to produce the gel-cell, polypyrrole and hydrogel, possess the ability to change configuration and produce movement [81, 141-143]. Harnessing their individual contractile qualities synergistically, hydrogel and polypyrrole could make a gel-cell contractile in response to activation. Next, by combining thousands of these miniatuarized contractile gel-cells through nanoimprint lithography [144], for example, they could be produced into a macroscopically contractile 3-D tissue and even an artificial heart (Figure 2.11, bottom left).

As a final example, the technology being developed here could be used in lightweight tubes capable of driving fluids as they propagate contractile waves down their length (Figure 2.11, bottom right). Peristaltic waves, similar to those produced by the intestines to move food through the digestive track, could propel fluid down the length of the tube. Such a technology could eliminate the need for heavy conventional 
pumps in space craft, where the need for lightweight materials and energy and space conservation are of prime importance.

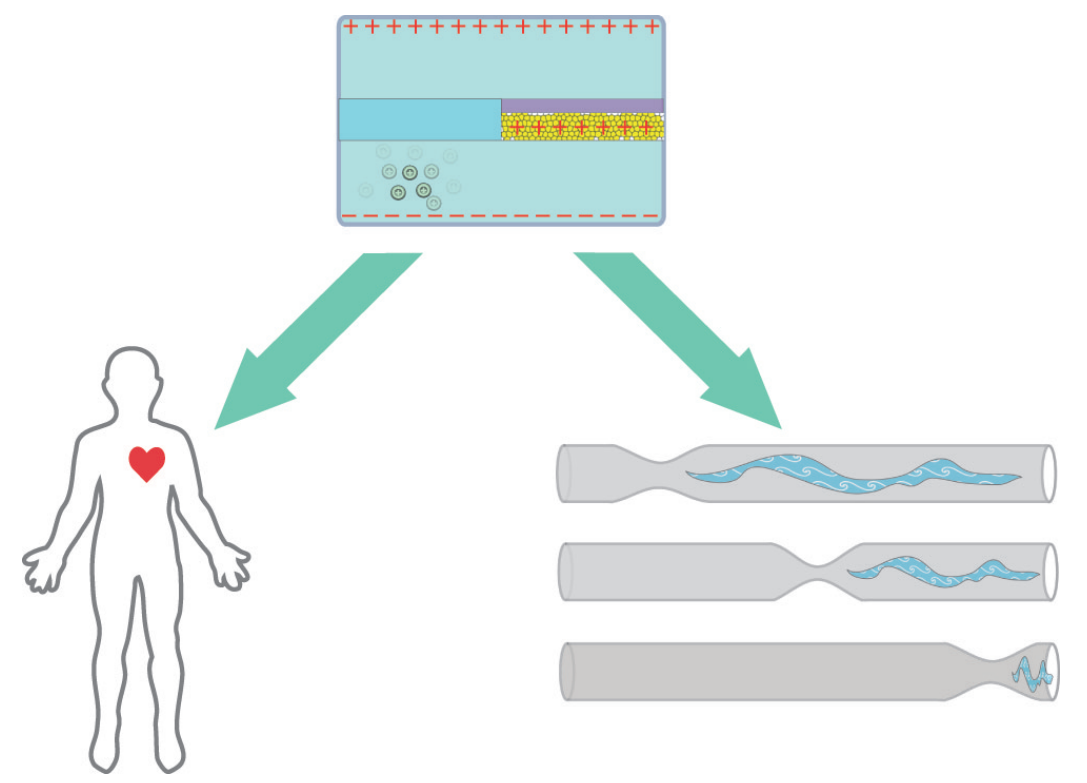

Figure 2.11: The gel-cell may some day be used to produce an artificial heart for the purpose of studying cardiac arrhythmias, or even serve as a replacement for a defective heart (bottom left). It may also be used as a tube pump where the artificial cells in an array are used to form the tube walls, and propagate a contractile wave down their length (bottom right). There are many more potential applications for this technology, as they could conceivably define a new class of artificial excitable contractile tissue. 


\section{Chapter 3}

\section{Methods}

In this chapter, methods for the preparation of both polypyrrole and hydrogel are presented. In addition, techniques employed for PPy thickness determination are discussed, along with an overview of potassium chloride assay methods in solution and hydrogel. Finally, experimental methods for the quantification of flux rates in both solution and hydrogel are shown.

\subsection{Polypyrrole}

Electropolymerization of polypyrrole involves four critical steps which are highly susceptible to variability. These steps are: monomer oxidization, radical-radical coupling, deprotonation, and chain propagation [145]. Since the morphology of the resulting membranes is highly variable and dependent on each of these steps, consistency in membrane preparation was necessary. Steps to provide reproducibility included the distillation of pyrrole monomer prior to experiments, deaeration during deposition, and temperature fixing by performing depositions on ice. These preparations assured, to the best of our ability, that resulting membranes were comparable. The details for membrane electropolymerization are described in more detail below. 


\subsubsection{Polypyrrole for Flux Experiments}

A membrane preparation made from polypyrrole and counterion p-toluenesulfonate $\left(\mathrm{TOS}^{-}\right)$was performed as described by Zhao et al. [146]. This particular recipe for polypyrrole was chosen for its demonstrated ability to gate potassium chloride ions in solution. The preparation included the use of distilled water as solvent, and an inexpensive stainless steel working electrode for PPy deposition. Stainless steel mirror finish plates were used to produce very thin membranes (on the order of a few microns), which could be peeled off easily and used free-standing in ion flux experimental setups.

\subsubsection{Materials}

Counterion p-toluenesulfonate sodium salt $\left(\mathrm{TOS}^{-}\right)$was used as received from VWR. Pyrrole, from Sigma-Aldrich, was distilled* and stored at $4{ }^{\circ} \mathrm{C}$ in a nitrogen $\left(\mathrm{N}_{2}\right)$ environment prior to use.

\subsubsection{Preparation}

A solution containing $0.2 \mathrm{M}$ pyrrole and $0.05 \mathrm{M} \mathrm{TOS}^{-}$in distilled water $\left(\mathrm{diH}_{2} \mathrm{O}\right)$ was dearated under $\mathrm{N}_{2}$ gas on ice for 30 minutes. A mirror finish stainless steel (\#304, 0.105" thick), obtained from McMaster-Carr, was used as the working electrode. The sheet was cut into smaller electrode pieces with surface area of $3.24 \mathrm{~cm}^{2}$, secured to a copper dipping electrode via double sided copper tape (Ted Pella, Inc.), and covered in insulating paint (XPS2000).

Depositions were carried out in a 3-electrode electrochemical cell system designed in the Soft Materials Lab (see Figure 3.1). A simple $60 \mathrm{ml}$ beaker was used as the bath, and a lid was designed in SolidWorks, then cut from a 5/8" thick black Delrin

\footnotetext{
${ }^{*}$ The pyrrole distillation process is noteworthy as it is hazardous. Pyrrole has a flash point of 36 ${ }^{\circ} \mathrm{C}$, and yet to be distilled its temperature must be elevated above a boiling point of $130{ }^{\circ} \mathrm{C}$. This must be done with caution, as an explosion will occur if the vapor concentration of pyrrole in air is between $3.1 \%$ and $14.8 \%$. Therefore, pyrrole solution must be slowly heated up while allowing pyrrole vapor to saturate the airspace above it before reaching the flash point.
} 
disk using a CNC Rapid Milling Machine from the Bio-Inspired Engineering Research Labs. The design of the lid allowed for the precise placement of the working electrode, reference electrode and counter electrode within the electrochemical cell, as well as a location for nitrogen gas to be supplied to the solution during deposition ${ }^{\dagger}$. The stainless steel electrode was used as a working / sense electrode, a saturated calomel electrode (SCE) as reference, and platinum mesh as counter electrode.

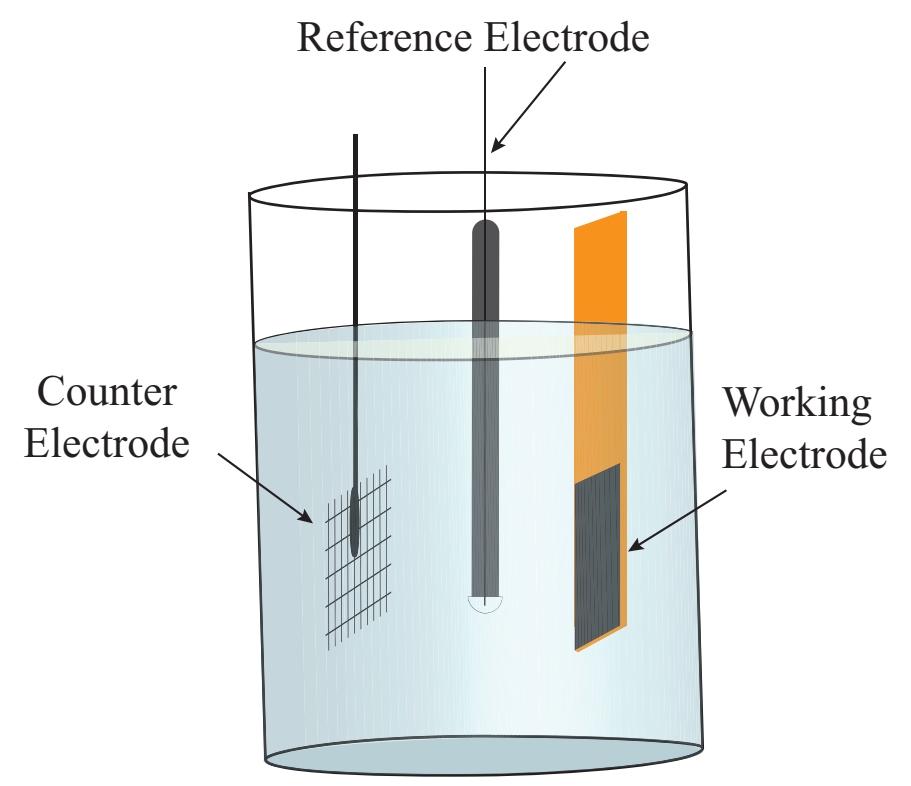

Figure 3.1: A three-electrode electrochemical cell used for the deposition of polypyrrole onto the working/sense electrode. A saturated calomel reference electrode and platinum mesh counterelectrode are needed to supply the appropriate voltage to the working electrode.

Galvanostatic depositions were carried out at $2 \mathrm{~mA} / \mathrm{cm}^{2}$ and $5 \mathrm{~mA} / \mathrm{cm}^{2}$ vs. SCE, using a VersaSTAT 3 potentiostat (Princeton Applied Research) for 5 to 35 minutes. Membrane survivability after detachment from the plate dictated a minimum of 15 minutes for deposition was required at $2 \mathrm{~mA} / \mathrm{cm}^{2}$ and 5 minutes at $5 \mathrm{~mA} / \mathrm{cm}^{2}$.

After deposition, PPy membranes were peeled off of the mirror finish working

\footnotetext{
${ }^{\dagger}$ In this arrangement, electrons are transferred between a working electrode upon which the polymer grows, and a platinum mesh counter-electrode placed some distance away from it. A third electrode, in this case a saturated calomel reference electrode, is placed in close proximity to both of these electrodes to provide an appropriate reading on the voltage drop existing between it and the working sense electrode connected in series to the working electrode. The placement of this reference electrode is critical as it dictates the appropriate voltage to be applied between the working and counter electrodes.
} 
electrodes as shown (see Figure 3.2), rinsed thoroughly in distilled water and stored in $\mathrm{diH}_{2} \mathrm{O}$ until needed. Flux experiments were typically performed immediately after depositions were completed, or the following day, as noted.

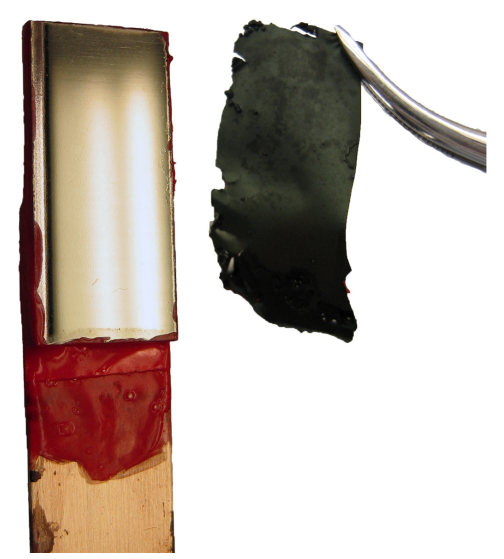

Figure 3.2: A mirror finish stainless steel \#304 electrode is shown. The resulting PPy from a galvanostatic deposition for 25 minutes at $0.5 \mathrm{~mA} / \mathrm{cm}^{2}$ can be easily peeled from the surface to produce a freestanding membrane, ready for use in flux experiments.

\subsubsection{Polypyrrole for Ion Selective Electrodes}

Although the $\mathrm{PPy} / \mathrm{TOS}^{-}$membranes described above are suitable for use in ion selective electrodes, it was found that preparations based on work by Tietje-Girault et al. resulted in stronger signal responses to potassium [147]. Their group used an acetonitrile $\left(\mathrm{CH}_{3} \mathrm{CN}\right)$ solvent instead of water, and new counterion tetrabutylammonium tetrafluoroborate $\left(\mathrm{Bu}_{4} \mathrm{NBF}_{4}\right)$. This recipe only successfully deposited onto noble metals, and for this reason, gold mesh was used as the working electrode instead of stainless steel.

\subsubsection{Materials}

Tetrabutylammonium tetrafluoroborate $\left(\mathrm{Bu}_{4} \mathrm{NBF}_{4}, 95 \%\right)$ from Acros Organics and acetonitrile $\left(\mathrm{CH}_{3} \mathrm{CN}\right)$ from Sigma-Aldrich were used as received and stored at room temperature. Pyrrole, also from Sigma-Aldrich, was distilled and stored at 4 ${ }^{\circ} \mathrm{C}$ in a nitrogen environment prior to use. Gold mesh was purchased from Unique 
Wire Weaving Co., Inc (www.uniquewire.com). Wire diameter was 0.0025", with 100 x 100 mesh size (56\% open area) and $99.99 \%$ purity.

\subsubsection{Preparation}

A solution containing $0.1 \mathrm{M}$ distilled pyrrole, $0.1 \mathrm{M} \mathrm{Bu}_{4} \mathrm{NBF}_{4}$, and $1 \%$ wt $\operatorname{diH}_{2} \mathrm{O}$ in acetonitrile was prepared and deaerated under nitrogen gas for 30 minutes on ice. The gold mesh working electrode was cleaned with ethanol, rinsed in $\operatorname{diH}_{2} \mathrm{O}$ and cut into $0.5 \mathrm{~cm}$ by $2 \mathrm{~cm}$ rectangles prior to use. A portion of the mesh was secured to the copper dipping electrodes using double sided copper tape, leaving a $0.5 \mathrm{~cm}$ by 1 $\mathrm{cm}$ area of the mesh extending beyond the electrode, which would be freestanding in the deposition solution. The copper tape and copper dipping electrode were covered in insulating paint, as shown in Figure 3.3. Using a VersaSTAT 3, potentiostatic depositions were carried out at $+0.8 \mathrm{~V}$ vs. SCE for 1 hour in the standard 3-electrode cell described above.

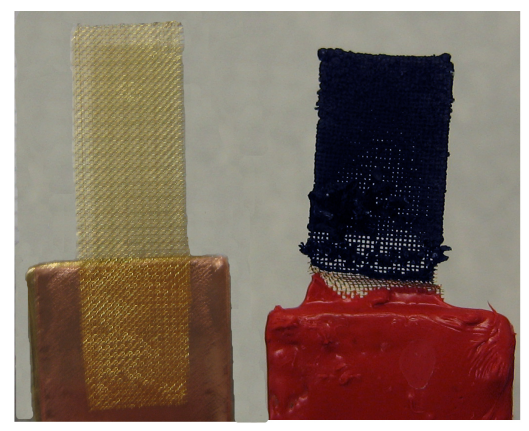

Figure 3.3: Gold mesh secured to the copper dipping electrode via double-sided copper tape (left). After deposition, PPy is shown on the gold mesh (right). These meshes do not represent the dimensions described above. However, they are representative of the gold mesh electrode attachment and painting process.

\section{$3.2 \quad$ PPy Thickness}

As membrane thickness directly influences the flux rate of potassium chloride by affecting the mean free path of travel for ions, a theoretical model predicting PPy membrane thickness was sought. Theoretically obtained values were then compared 
to experimentally determined thickness values to test the model validity. The methods for both theoretical and experimental thickness determination are demonstrated below.

\subsubsection{Theoretical Prediction}

Theoretical predictions of PPy membrane thickness were computed based on work by Farrington et al. [148], whose model relates the membrane thickness $(d)$, to the charge accumulation $(Q)$ during deposition, as follows:

$$
d=\frac{Q}{n \cdot F \cdot A \cdot \rho}\left[M_{1}+\frac{M_{2}}{y}\right] .
$$

Membrane thickness values were calculated for PPy / TOS $^{-}$membranes (as prepared for flux experiments), with $n$ being the number of electrons, 2 , involved in the polymerization process. $F$ is the Faraday constant, and $A$ is the surface area of the electrode onto which PPy is deposited. The PPy film density, $\rho$, was approximated as $1.1 \mathrm{~g} / \mathrm{cm}^{2}$ by Farrington et al. [148]. The number of pyrrole units per counterion, $y$, was also approximated at 3 by their group. $M_{1}$ and $M_{2}$ are the molecular weights of pyrrole and counterion $\mathrm{TOS}^{-}$, respectively. The charge density $(Q)$ for deposition was calculated from the current density applied at the membrane $(J)$, the working electrode surface area $(A)$, and duration of the deposition $(t)$ according to Equation 3.2 below:

$$
Q=J \cdot A \cdot t
$$

Results obtained from this model are presented and discussed further in the results, Chapter 4. 


\subsubsection{Scanning Electron Microscopy}

Cross-sectional images of the membranes were taken using a scanning electron microscope (SEM, JEOL JSM-6700F cold field-emission gun). After deposition and in preparation for imaging, membranes were covered with $120 \stackrel{\circ}{A}$ of gold-palladium to increase conductivity and reduce membrane charging during imaging, using a Precision Etching Coating System (PECS, Gatan 682) located in the Nanoscale Materials Characterization Facility. Membranes were then mounted on a 90-degree angle vertical bore SEM specimen mount using double-sided carbon tape (Ted Pella, Inc.). Crosssectional views of each membrane were analyzed using the measurement tool within the JEOL software to determine exact thickness. An example SEM cross-sectional view is shown in Figure 3.4, below. Results obtained will be further discussed in Chapter 4 .

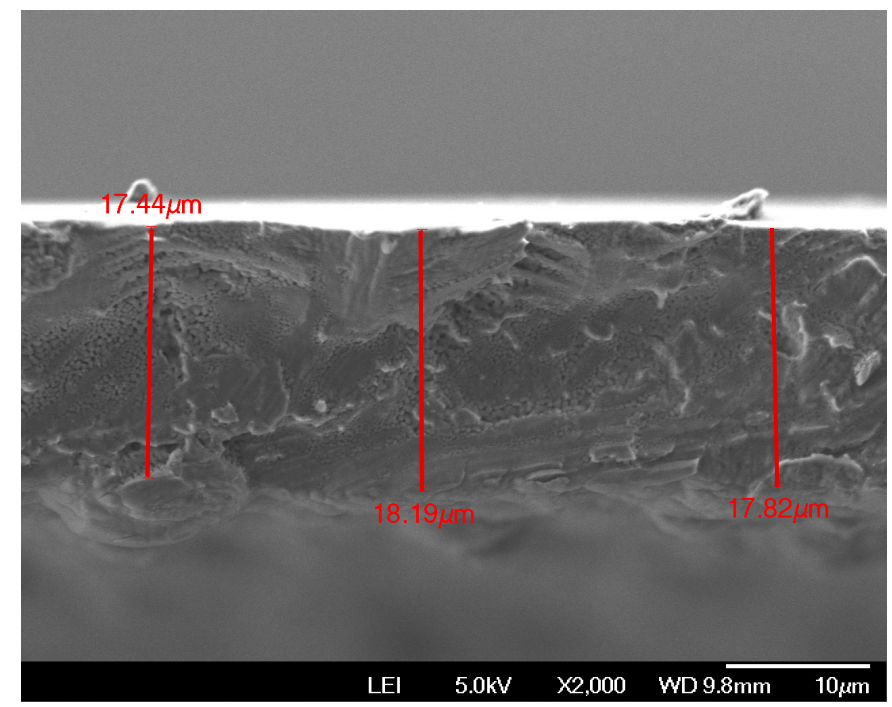

Figure 3.4: The cross-sectional view at 2000X of a $\mathrm{PPy} / \mathrm{diH}_{2} \mathrm{O}$ membrane (galvanostatic deposition at $2 \mathrm{~mA} / \mathrm{cm}^{2}$ for 35 minutes) is shown, taken on a JEOL $6700 \mathrm{~F}$ cold field-emission gun SEM. The instrument's software measurement tool was used to sample three locations across the membrane for an average thickness reading. 


\subsection{Hydrogel}

Hydrogels possess many qualities that make them attractive for use in the gel-cell. Among others, their high water content, excellent ion mobility within their matrix, and proven compatibility with polypyrrole motivated their selection. Preparation was based on a recipe from Barthus et al., which originally used the hydrogel as a support matrix for the growth of polypyrrole [114].

\subsubsection{Materials}

All materials were used as received. N,N'-methylene-bis-acrylamidde (MBA) from Sigma-Aldrich, and acrylamide from Cole-Palmer, were stored at $4{ }^{\circ} \mathrm{C}$ upon reception. N',N,N',N'-tetramethylethylenediamine (TEMED), also received from Sigma-Aldrich, was stored at room temperature. Potassium peroxodisulfate (PTS, 99\%) from VWR International was stored at room temperature, and capped under nitrogen atmosphere.

\subsubsection{Preparation}

Hydrogels of different pore sizes were prepared by mixing varying ratios of MBA to acrylamide, known as cross-linker ratios, into $8 \mathrm{ml}$ of distilled water $\left(\mathrm{diH}_{2} \mathrm{O}\right)$. Mixtures were then deaerated under nitrogen gas for 10 minutes, followed by the addition of $10 \mu \mathrm{L}$ TEMED and $10 \mathrm{mg}$ PTS. Hydrogel pore sizes of $2 \mathrm{~nm}$ and 0.52 nm, provided in Table 3.1, are based on work by Barthus et al. [114]. The smallest pore size, $0.1 \mathrm{~nm}$, was approximated by extrapolation from the two other known pore sizes.

The final solution was then placed into molds and left to gel for approximately 30 minutes. Once removed from the molds, hydrogels were soaked in $\mathrm{diH}_{2} \mathrm{O}$ to remove any impurities for over 24 hours. 
Table 3.1: Hydrogel Composition Ratios with Resulting Pore Sizes

\begin{tabular}{|c|c|c|c|}
\multicolumn{1}{c}{} & \multicolumn{3}{c}{ Hydrogel Compositions } \\
\hline MBA & $0.020 \mathrm{mg}$ & $0.044 \mathrm{mg}$ & $0.044 \mathrm{mg}$ \\
\hline Acrylamide & $0.418 \mathrm{mg}$ & $0.816 \mathrm{mg}$ & $0.676 \mathrm{mg}$ \\
\hline Cross-linker Ratio & 0.048 & 0.054 & 0.065 \\
\hline Pore Size & $2 \mathrm{~nm}$ & $0.52 \mathrm{~nm}$ & $0.1 \mathrm{~nm}$
\end{tabular}

\subsection{Potassium Chloride Detection}

To quantify the presence of potassium chloride in hydrogels, and ultimately within the gel-cell, two ion measurement methods were employed. The first, adapted from known electrochemical impedance spectroscopy (EIS) methods and performed via a customized probe, was developed to detect real-time and direct measurements of the salt in solution and in hydrogel. Although this method was highly successful and provided valuable information for the flux rate calculations, the complexity of the data capture system (involving the use of a potentiostat) made the technique limited in application for the gel-cell.

A secondary detection method was therefore tested, which involved the use of potassium sensors based on polypyrrole, as developed by Pandey et al. [138]. This ion-selective electrode (ISE) method for detecting potassium was more simple to run than the EIS probe, and is anticipated for use in the final gel-cell design.

The preparation and methods for these two detection systems (EIS probe and ISE sensor) are described in detail below, with results obtained from these systems discussed further in Chapter 4.

\subsubsection{Electrochemical Impedance Spectroscopy}

\subsubsection{Materials and Preparation}

Electrochemical impedance spectroscopy (EIS) probes were prepared using two platinum squares (each $0.5 \mathrm{~cm}$ by $0.5 \mathrm{~cm}$, and 0.004" thick, from ESPI Metals) in parallel and $0.5 \mathrm{~cm}$ apart from one another (see Figure 3.5). Electrical wires (14 
gauge) were soldered onto the back sides of the platinum electrodes and covered in XPS2000 insulating paint. The two electrodes were held parallel and in place by a custom designed U-shaped plastic guide (not shown).

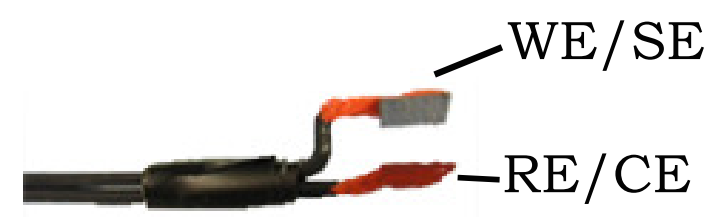

FigURE 3.5: An electrochemical impedance spectroscopy (EIS) probe made from two parallel platinum squares is used to quantify the resistance of the material between it's leads (solution or hydrogel), which is calibrated to represent ion concentration.

The working / sense leads of a VersaSTAT 3 potentiostat were connected to one platinum electrode, and the counter / reference to the other. An alternating current $2 \mathrm{mV}$ in magnitude and $0 \mathrm{~V}$ DC offset was applied to the platinum electrodes, while sweeping the AC frequency from $150 \mathrm{kHz}$ to $0.1 \mathrm{~Hz}$. The resulting real and imaginary impedances were used to gain information about the resistivity of the solution (or hydrogel) present between the probes.

The EIS probe response behavior can be described by the equivalent electrical circuit seen in Figure 3.6. The equivalent circuits for the surfaces of the platinum plates, which were confirmed by observing the bode response of an impedance sweep applied through the system, are represented by the capacitors and resistors in parallel, $\mathrm{R}_{1} / \mathrm{C}_{1}$, and $\mathrm{R}_{2} / \mathrm{C}_{2}$. The resistivity of the solution between the probe plates is represented by a resistor, $\mathrm{R}_{S}$, which will vary as a function of ion concentration, and can be determined via impedance measurement of the system. 


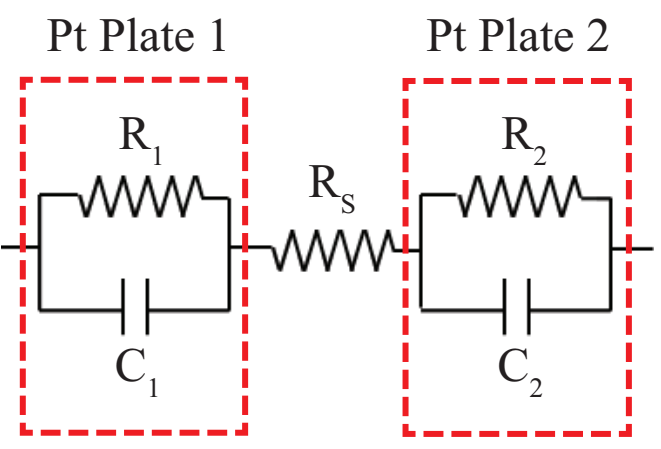

FiguRE 3.6: Equivalent electrical circuit of the EIS probe system. Each platinum electrode is represented by a resistor and capacitor in parallel. Resistor $R_{S}$ represents the solution between the electrodes. As described below, the concentration of ions in solution can be determined in the solution from this equivalent circuit using impedance spectroscopy to relate solution resistivity to ion concentration.

A frequency sweep of alternating current is applied to the circuit of the EIS probes via the potentiostat, from 0.1 to $150 \mathrm{kHz}$. At high frequencies, capacitors act as electrical short-circuits in the above equivalent circuit, revealing only the value of $\mathrm{R}_{S}$ in the complex impedance response. As seen in Figure 3.7 below, when placed in solution, the EIS probe produces a linear response to varying concentrations of $\mathrm{KCl}_{a q}$ in the high frequency domain, starting above approximately $3000 \mathrm{~Hz}$. This allowed the probe to be sampled only at a high frequency $(150 \mathrm{kHz})$ repeatedly, to obtain timevarying concentrations of $\mathrm{KCl}_{a q}$. This single frequency impedance approach became critical for the time-resolved data obtained during membrane flux experiments, as will be discussed in the following Chapter 4. Calibration curves in both solution and hydrogels are presented and discussed further in the following Chapter 4, as well. 


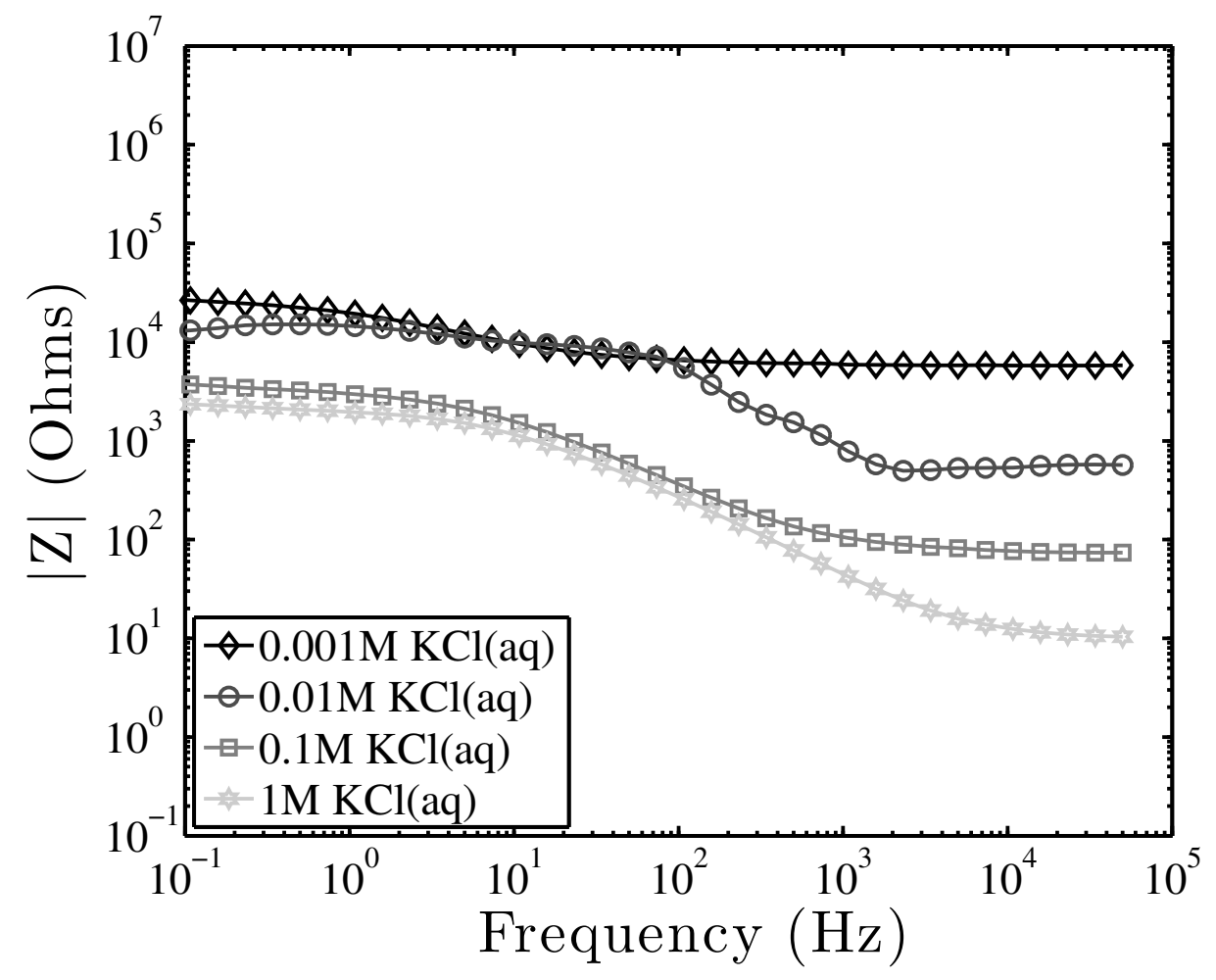

FIGURE 3.7: Electrochemical impedance spectroscopy response of the probes showing the impedance magnitude $(|\mathrm{Z}|)$ over the frequencies tested. The EIS probe is in aqueous solutions of $\mathrm{KCl}$ ranging from $0.001 \mathrm{M}$ to $1 \mathrm{M}$. In the high frequency regime, near $60 \mathrm{kHz}$, the average $|\mathrm{Z}|$ response represents the $\mathrm{R}_{S}$ value of the solutions tested. A linear response to $\mathrm{KCl}_{a q}$ concentrations is observed in this region.

\subsubsection{Ion Selective Electrode}

\subsubsection{Materials and Preparation}

Potassium ion selective electrodes (ISE) were produced according to Pandey et al. by using an ionophore coating to amplify the innate potassium detection ability of PPy [138]. PPy membranes were prepared as described above in acetonitrile base (see Section 3.1.2) on a gold mesh working electrode. Immediately following deposition, the membrane was rinsed in acetonitrile and dried under a stream of $\mathrm{N}_{2}$ gas. Meanwhile, a mixture of $56 \mathrm{mg}$ of poly(vinyl chloride) (PVC, Sigma-Aldrich) powder and $1.2 \mathrm{ml}$ tetrahydrofuran (THF, Sigma-Aldrich) was combined and set aside $\left(\mathrm{S}_{1}\right)$. A second solution $\left(\mathrm{S}_{2}\right)$ was prepared with $2 \mathrm{mg}$ of dibenzo-18-crown-6, $0.24 \mathrm{ml}$ of dibutyl phthalate, and $1.5 \mathrm{mg}$ of tetraphenyl borate (also from Sigma-Aldrich). After 
mixing thoroughly, $0.12 \mathrm{ml}$ of $\mathrm{S}_{2}$ was added to $\mathrm{S}_{1}$. The PPy membrane was then covered with 8 drops of this mixture and left to dry for 15 minutes. This was repeated a total of three times on each side of the membrane, after which it was conditioned in $0.01 \mathrm{M} \mathrm{KCl}_{a q}$ for 20 hours.

In the presence of potassium ions, the ISE sensor produces a voltage drop with respect to a reference electrode which is proportional to the ion concentration. To test the sensor, an apparatus for dipping the ISE in potassium chloride was setup as shown in Figure 3.8. A PEEK frit (2 $\mu \mathrm{m}$ pore size, Upchurch Scientific) separated the test solution from a potassium phosphate buffer solution ( $\mathrm{pH} 7$, Fisher Chemical), in which a customized silver / silver chloride reference electrode ${ }^{\ddagger}$ was placed. A voltage drop was read across this reference electrode and the ISE as shown in Figure 3.8, via LabVIEW using a Data Acquisition (DAQ) device.

\footnotetext{
${ }^{\ddagger}$ The silver chloride electrode was made from small glass tubes (3 $\mathrm{mm}$ OD) inserted with a silver wire from VWR International. The silver wire was treated in chlorox for several hours before use. A $3 \mathrm{~mm}$ OD frit (from Upchurch Scientific) was secured to the tip of the glass tube using heat shrinkable tubing, and filled with $1 \mathrm{M} \mathrm{KCl}_{a q}[149]$.
} 


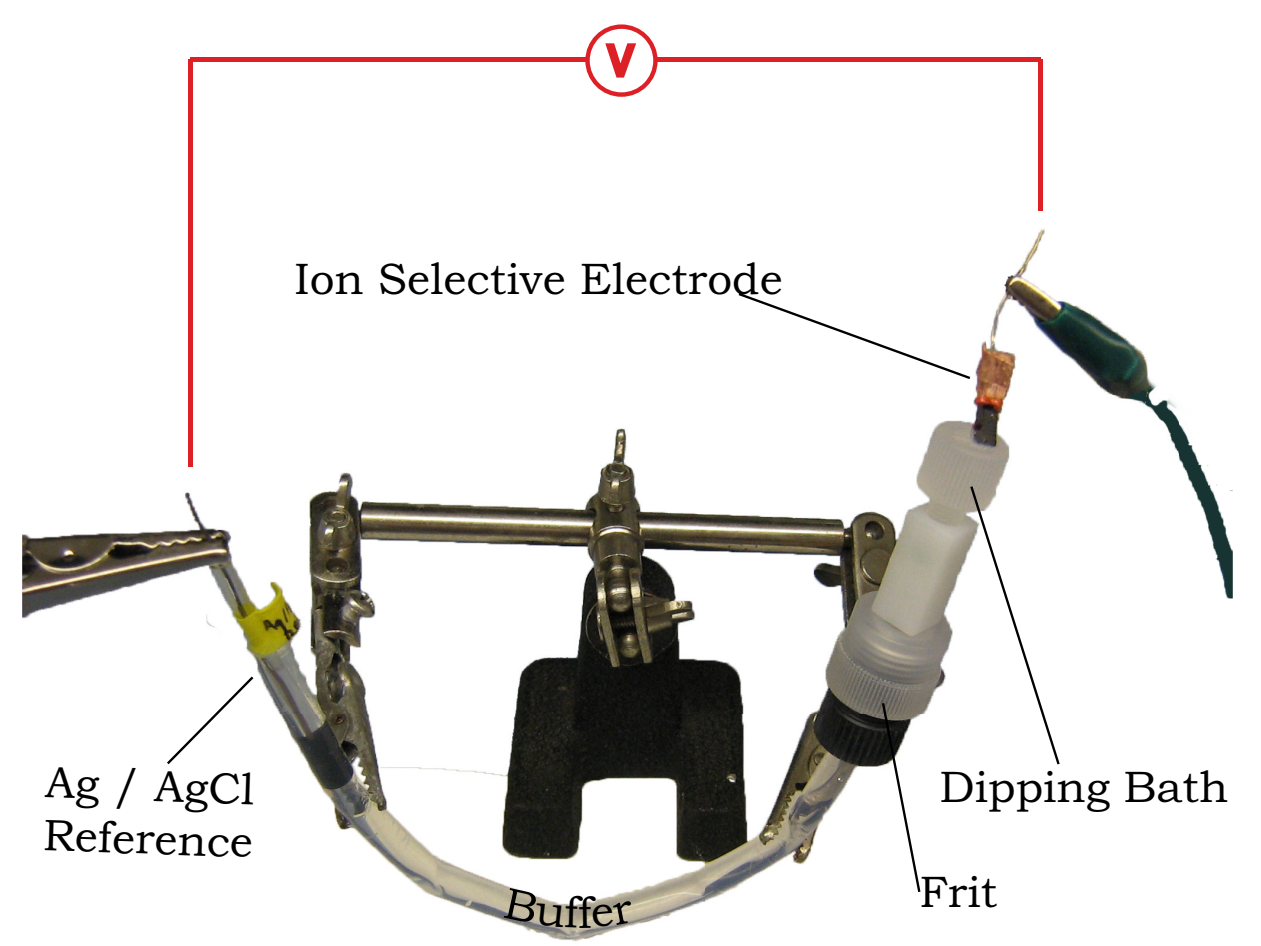

Figure 3.8: The testing setup for the ion selective electrode is shown. A dipping bath on the right hand side is filled with a potassium testing solution for the ISE. A frit separates the testing solution from a buffer solution ( $\mathrm{pH} 7$ ) in which the in-house $\mathrm{Ag} / \mathrm{AgCl}$ reference electrode is placed. Using alligator clips to interface with the system, voltage drop readings across the ISE and reference electrode are measured via a DAQ card and recorded via LabVIEW.

The ISE sensor was also used to detect the presence of potassium ions in hydrogel. Sensing electrodes were prepared as described above and encapsulated in a cylindrical hydrogel, to allow a direct contact between the hydrogel and the electrode surface (Figure 3.9). The hydrogel / sensor combinations were then soaked twice in $\operatorname{diH}_{2} \mathrm{O}$ as a pre-treatment, and tested in increasing concentrations of $\mathrm{KCl}_{a q}$, starting at $\operatorname{diH}_{2} \mathrm{O}$ and ending at $1 \mathrm{M} \mathrm{KCl}_{a q}$. Calibration curves for the sensing electrode were produced by recording voltage readouts as a function of ion concentration, and compared to sensor results in solution, as shown below in Figure 3.10. It is interesting to note that the sensor response in hydrogel offers an improved sensitivity over solution at higher concentrations of $\mathrm{KCl}_{a q}$. 


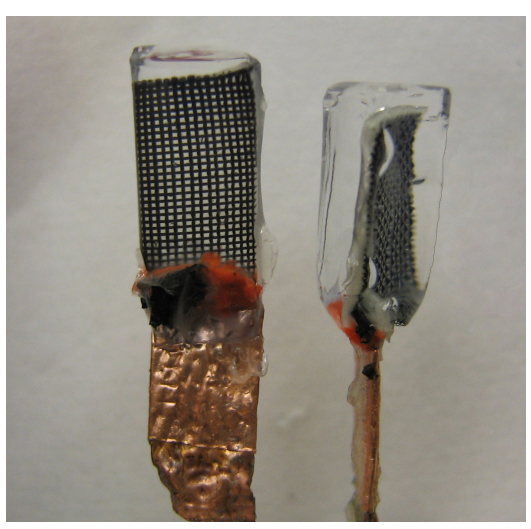

Figure 3.9: Two sensing electrodes produced in the Soft Materials Lab at UVa are shown side by side. They have been covered in a cylindrical hydrogel of $2 \mathrm{~nm}$ pore size.

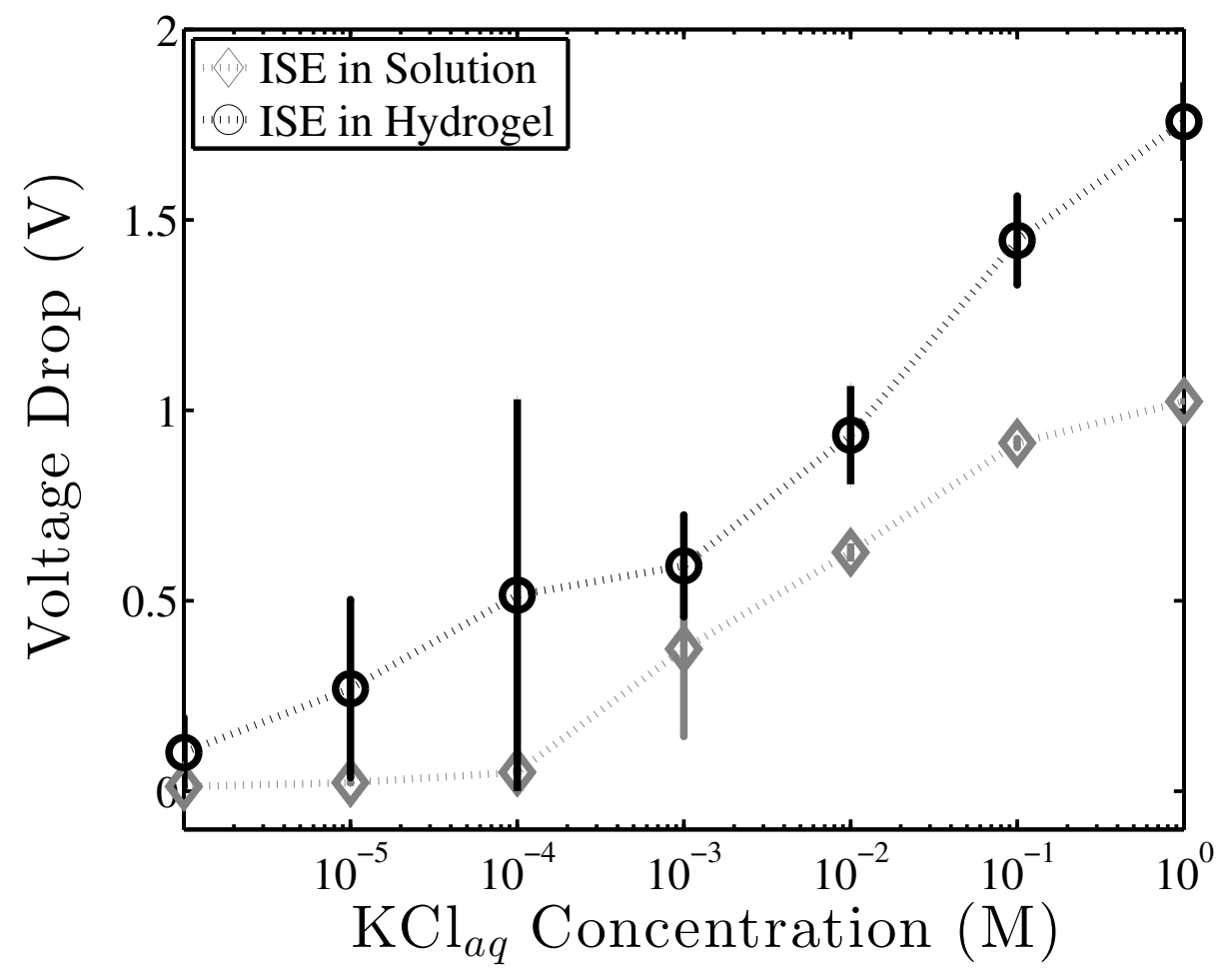

FiguRE 3.10: The voltage drop is shown for ISE sensors in solution and hydrogel as a function of $\mathrm{KCl}_{a q}$ concentration. The averaged response of two potassium sensing electrodes for each curve is shown. From these data it can be seen that the sensor response in hydrogel offers an improved sensitivity over solution at higher concentrations of $\mathrm{KCl}_{a q}$. 


\subsection{Flux Rate Measurements}

The flux measurement of potassium ions through polypyrrole was performed in a two-chamber transport cell designed in the Soft Materials Lab. The flux cell was designed to hold a PPy membrane in place hermetically between two solutions, while in contact with an electrode capable of supplying potentials to modulate its conductive states. Experiments were inspired by the work of Mirmohseni [150] and Partridge et al. [151]. Details of the flux cell are given below, followed by procedures for flux rate measurement.

\subsubsection{Flux Cell}

A PPy membrane was prepared as described in Section 3.1.1 above, on a stainless steel plate and rinsed in $\mathrm{diH}_{2} \mathrm{O}$ following deposition. It was then placed against a thin platinum $(\mathrm{Pt})$ ring working electrode, and secured in place between two halves of a flux apparatus by a C-clamp (see Figure 3.11). The platinum ring electrode (0.01" thick) was custom prepared by Precision Tool \& Die for the flux cell according to the design shown in Figure 3.12, and the flux cell was cut from a piece of white Delrin (McMaster-Carr) according to the design shown in Figure 3.13. 


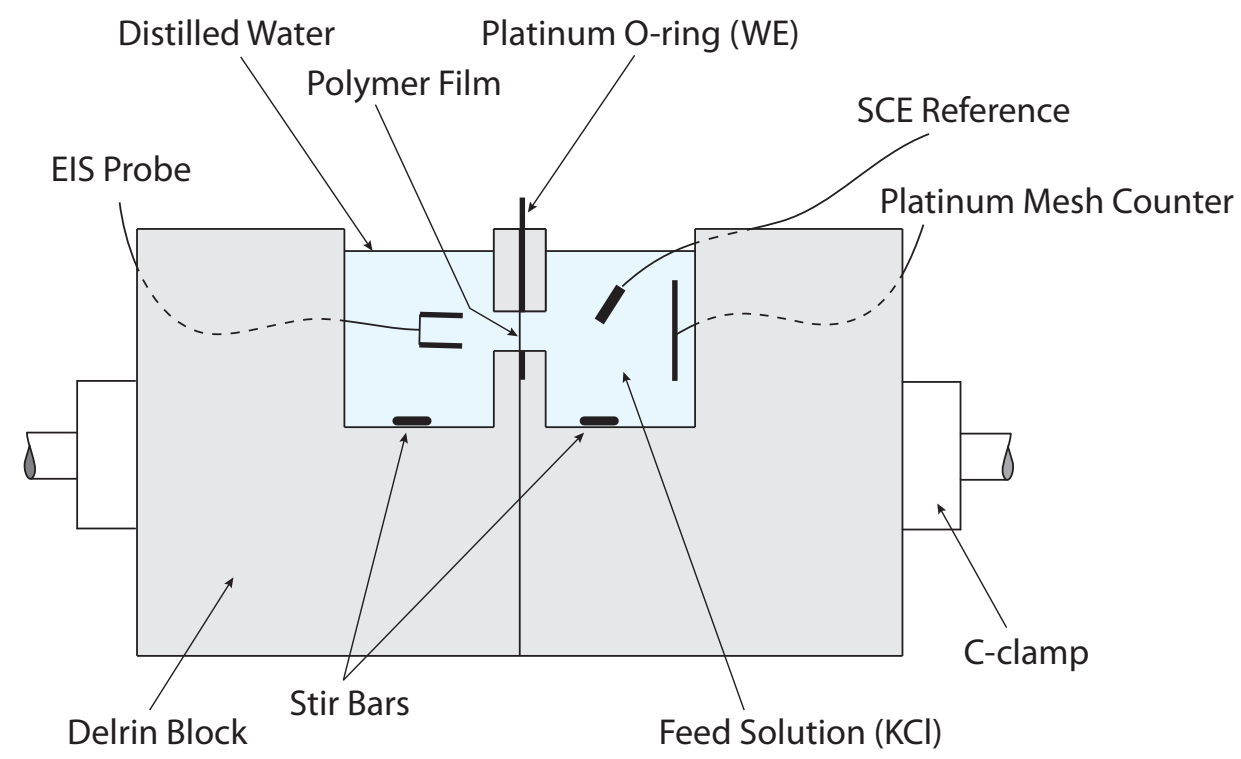

Figure 3.11: Schematic of flux cell layout. Two delrin pieces are held together by a C-clamp, which maintains the PPy membrane tightly in place between the two chambers and in contact with the platinum ring electrode. A feed solution containing high concentrations of $\mathrm{KCl}_{a q}$ is on the right and a receiving solution containing $\mathrm{diH}_{2} \mathrm{O}$ is on the left. An EIS probe is placed in the receiving solution and connected to a potentiostat. The feed solution contains the platinum mesh counter and SCE reference electrodes, also connected to a potentiostat. Micro stir bars are placed in both chambers.
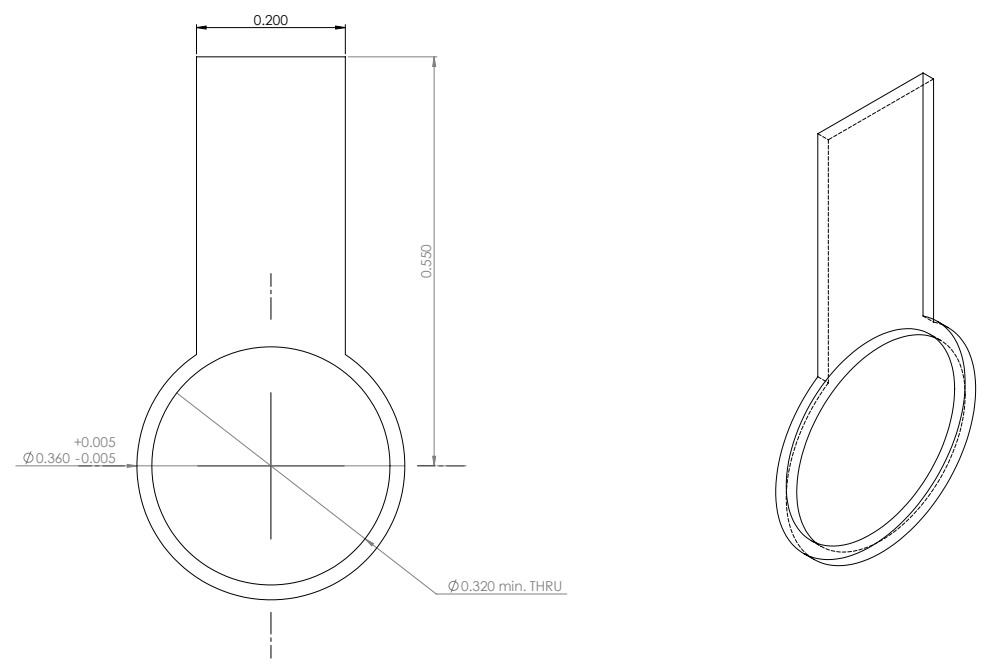

FIGURE 3.12: Schematic of the platinum ring electrode which contacts the PPy membrane supplying it with current. The electrode was produced by Precision Tool \& Die. 


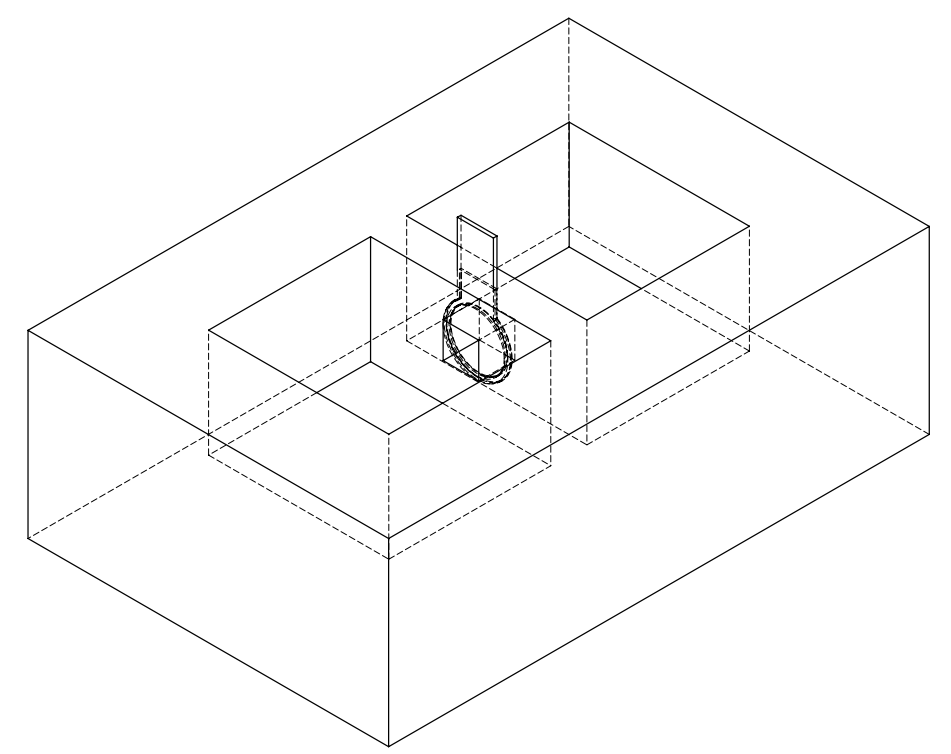

FIGURE 3.13: Schematic representation of the flux apparatus including positioning of the platinum ring working electrode, against which the PPy membrane is placed. The piece was cut from Delrin and the platinum ring was secured via a thin strip of Scotch Tape 3M.

A potassium ion gradient was created across the membrane in order to observe ion transport, by filling one bath with $1 \mathrm{M} \mathrm{KCl}_{a q}$, and the other with $\operatorname{diH}_{2} \mathrm{O}$. Within the source bath of $1 \mathrm{M} \mathrm{KCl}_{a q}$, a saturated calomel reference electrode (Accumet) and a customized platinum mesh counter electrode were placed, as shown in Figure 3.14. Micro stirring bars (Fisherbrand, Fisher Scientific) were placed in each well to agitate the solutions. In the receiving bath containing $\mathrm{diH}_{2} \mathrm{O}$, an EIS probe is placed to quantify the presence of potassium ions as they move through the membrane. The Pt ring was used as the working electrode (WE), a saturated calomel electrode as reference (RE), and a platinum mesh electrode as counter (CE), and all interfaced with a potentiostat. 


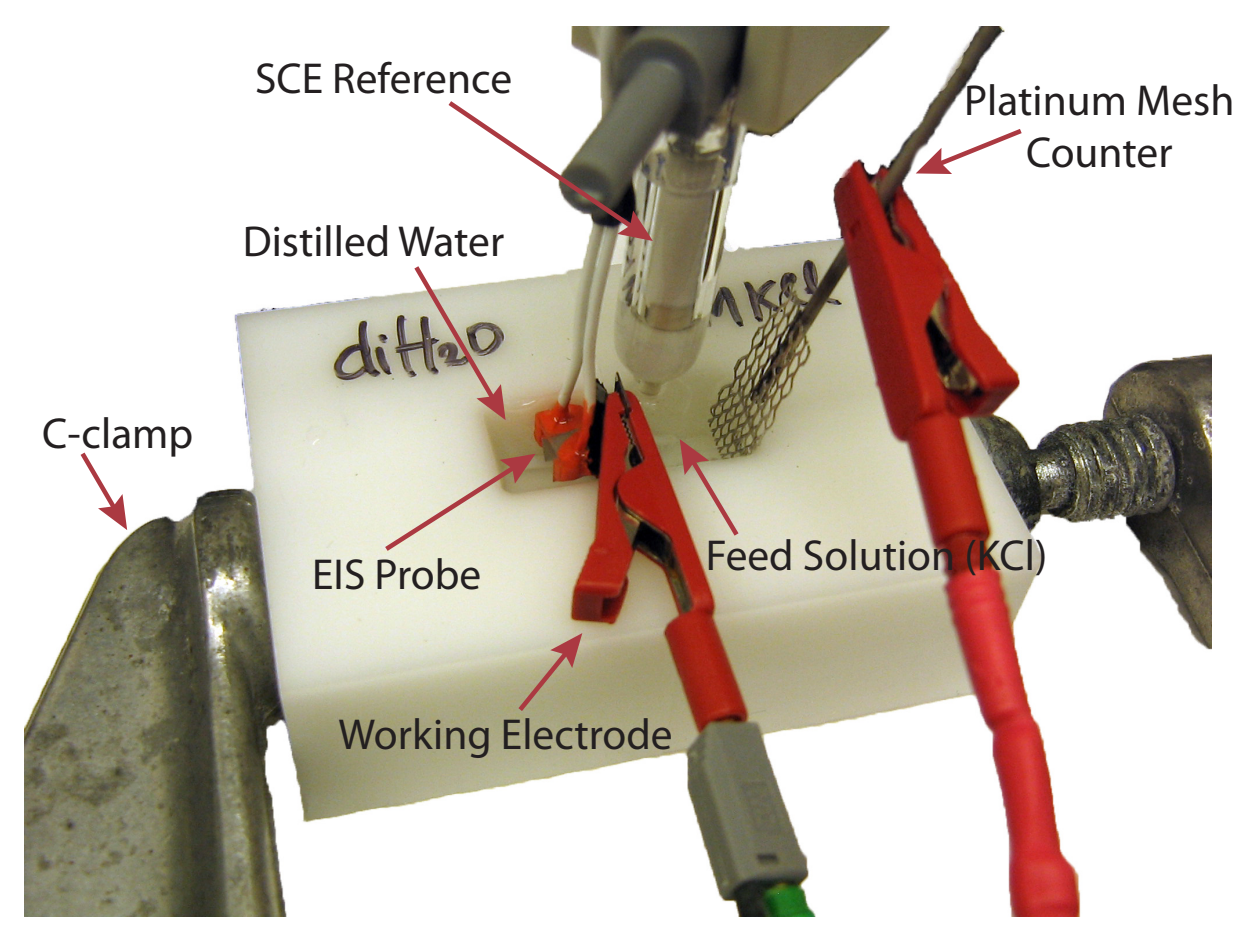

FiguRE 3.14: Picture of the flux cell showing the emplacement of the EIS probe, SCE reference and platinum mesh counter electrodes relative to the working electrode. Also shown is the C-clamp holding the two sides of the flux cell together.

\subsection{2 'Time-Resolved' versus 'Time-Lapse' Measurements}

Measurement of potassium chloride flux rates through PPy was performed in either of two ways, as depicted in Figure A.9 below. The first method, time-resolved data capture, involved the use of two potentiostats working in parallel during the flux experiments. One instrument was used to capture the EIS probe concentration readouts, while the second was used to apply the various potentials at the membrane to modulate its conductivity. This allowed a continuous read-out on the potassium concentration in the receiving bath, and provided valuable information for the short term flux kinetics through the membrane, as will be discussed more in the Results, Chapter 4 . 


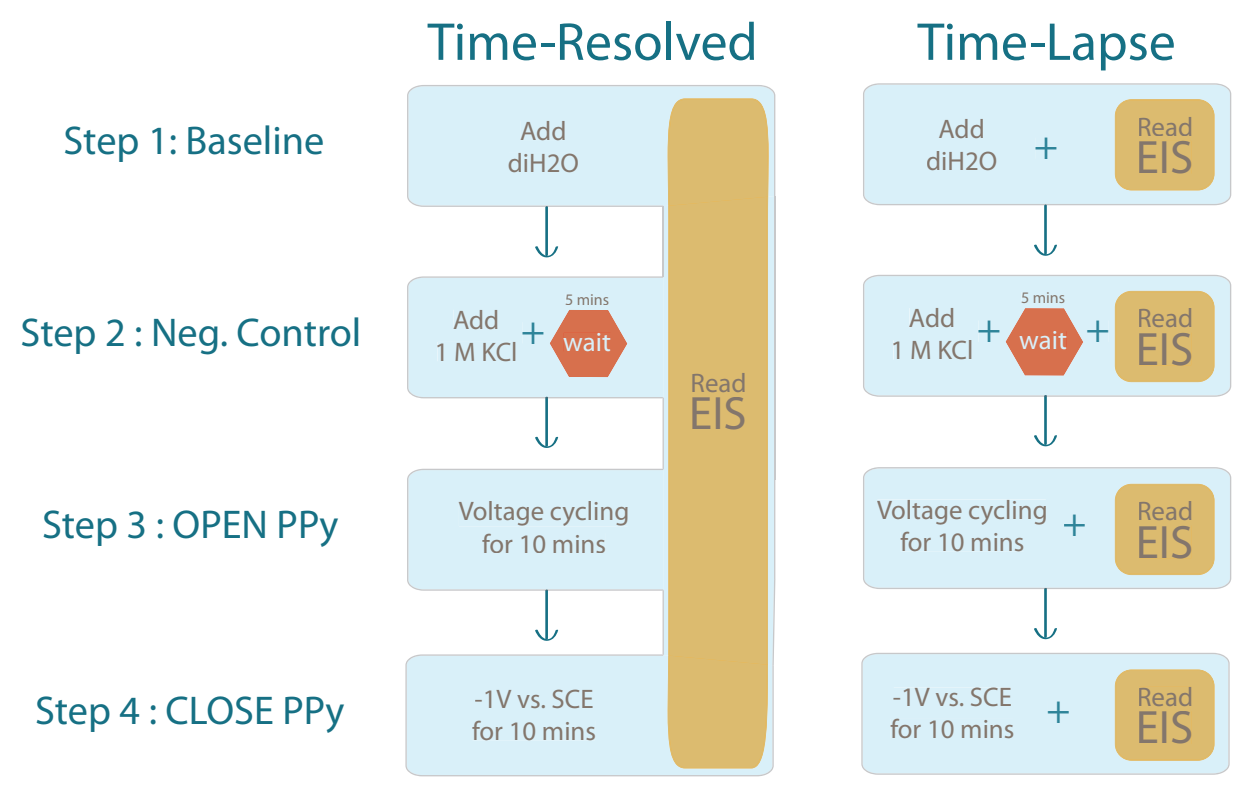

FiguRE 3.15: Flux experiment sequence, shown for both time-resolved and time-lapse capture modes. The two approaches proceed identically through steps $1-4$, however in the time-resolved case a continuous read-out of the $\mathrm{KCl}_{a q}$ concentration is provided by the EIS probe, since two potentiostats are used in parallel. In the case of time-lapse measurements, an EIS reading is performed after each step instead, using a single potentiostat. Step 1: a baseline EIS reading is performed on diH ${ }_{2} \mathrm{O}$. $\mathrm{Step}$ 2: a negative control reading is taken after a 5 minute wait post $\mathrm{KCl}_{a q}$ addition. Step 3: the PPy membrane is opened by cycling voltages across it for 10 minutes, Step 4: the membrane is closed.

The second method, involving time-lapse data capture, proceeded through the exact same sequence of steps at the membrane, however, this time in the presence of a single potentiostat for data capture. This method was developed to circumvent feedback loops occurring between two potentiostats when time-resolved data capture was performed on PPy / hydrogel sandwich structures. The time-lapse data capture was performed, as shown in Figure A.9, by following the same sequence of steps, however, this time the same potentiostat would be disconnected from the three electrodes controlling the membrane oxidation state, and attached to the EIS probe to perform 2 minutes of concentration reading.

The sequence of steps applied at the membrane were: 1) a baseline EIS probe reading on $\operatorname{diH}_{2} \mathrm{O} ; 2$ ) adding $1 \mathrm{M} \mathrm{KCl}_{a q}$ to the source side well, a 5 minutes wait, and a second EIS reading; 3) cycling oxidizing / reducing potentials on the membrane for 10 minutes at $\pm 1 \mathrm{~V}$ vs. SCE with 50 second pulse width (unless otherwise speci- 
fied), immediately followed by an EIS probe concentration reading; and 4) applying a reducing potential (which closes the membrane to ion flux) at the membrane for another 10 minutes, and taking a final EIS reading. The EIS probe measurements described were always performed continuously at $150 \mathrm{kHz}$ AC for 2 minutes. Results obtained from these experiments will be discussed further in the following Chapter 4.

\subsubsection{Flux through PPy / Hydrogel}

Preparation of a PPy / hydrogel structure (as shown in Figure 3.16) for flux experiments was performed as described here. After PPy deposition, hydrogels were added on both sides of the membrane via cubic molds with $0.5 \mathrm{~cm}$ side dimensions. Once the hydrogels were solidified on the membrane, the PPy / hydrogel sandwich structures were carefully extracted from the mold, and soaked in $\mathrm{diH}_{2} \mathrm{O}$ overnight. The membrane / hydrogel assemblies were then secured in the flux apparatus as shown in Figure 3.17.

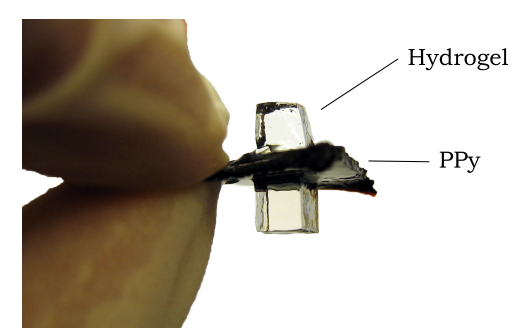

Figure 3.16: A PPy membrane with encapsulating hydrogels, as prepared for placement in the flux cell. The hydrogels were added to the PPy membrane using a cubic mold with $0.5 \mathrm{~cm}$ sides. 


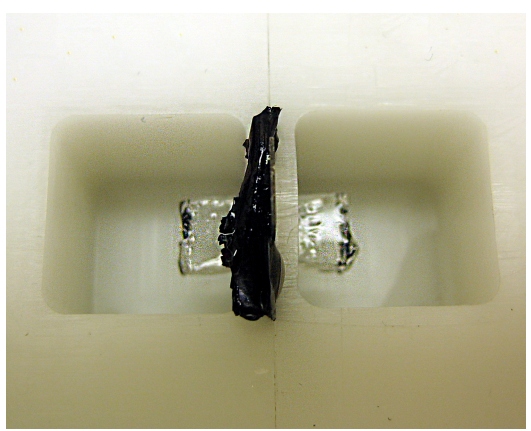

Figure 3.17: A PPy / hydrogel sandwich structure is shown wedged between the two chambers of a flux cell. The hydrogel protrusions fit snugly within the openings of the chambers.

The time-lapse flux measurement method was applied to the PPy / hydrogel sandwich structure for data acquisition, as described in Figure A.9. The only difference from before being the EIS probe placement directly on the hydrogel, as the EIS probe width was the same as that of the hydrogel. Results for these experiments will be described in detail in the next chapter. 


\section{Chapter 4}

\section{Results}

The results presented here have been divided into four main sections for clarity. The first section covers the characterization of polypyrrole membranes; via cyclic voltammetry, imaging, thickness and density determinations. The second section covers the characterization of hydrogel; its water content, and electrochemical impedance spectroscopy calibration response. Thirdly, flux experiments in solution and through PPy/hydrogel structures are shown. And lastly, the detection of potassium ions via ISE is demonstrated in hydrogels.

\subsection{Polypyrrole}

Polypyrrole membranes have been successfully produced and characterized experimentally via cyclic voltammetry, scanning electron microscopy, and gas pycnometry to determine criteria meaningful for their incorporation into the gel-cell design. The electroactive polymer's ability to gate ions was observed and quantified in both solution and hydrogel, achieving high ionic flux rates in both of these media, as shown in more detail in the sections below. 


\subsubsection{Cyclic Voltammetry}

Immediately following the deposition of PPy onto a stainless steel plate working electrode (as described in Section 3.1.1 above), membranes still on the working electrode were rinsed in $\mathrm{diH}_{2} \mathrm{O}$ and placed in a three-electrode cell setup where they were characterized via cyclic voltammetry. A linear voltage sweep from $-1 \mathrm{~V}$ to $+1 \mathrm{~V}$ vs. reference electrode was applied at the membrane for a total of 10 cycles at $100 \mathrm{mV} / \mathrm{s}$ $[152,153]$. As denoted in Figure 4.1 below, positive voltages at the membrane oxidize the polypyrrole, while negative ones reduce it. The membranes were characterized in either 1,2 or $4 \mathrm{M} \mathrm{KCl}_{a q}$ electrolyte solution. The currents obtained from these varying potentials give information on the reaction kinetics between the membrane and $\mathrm{KCl}_{a q}$.

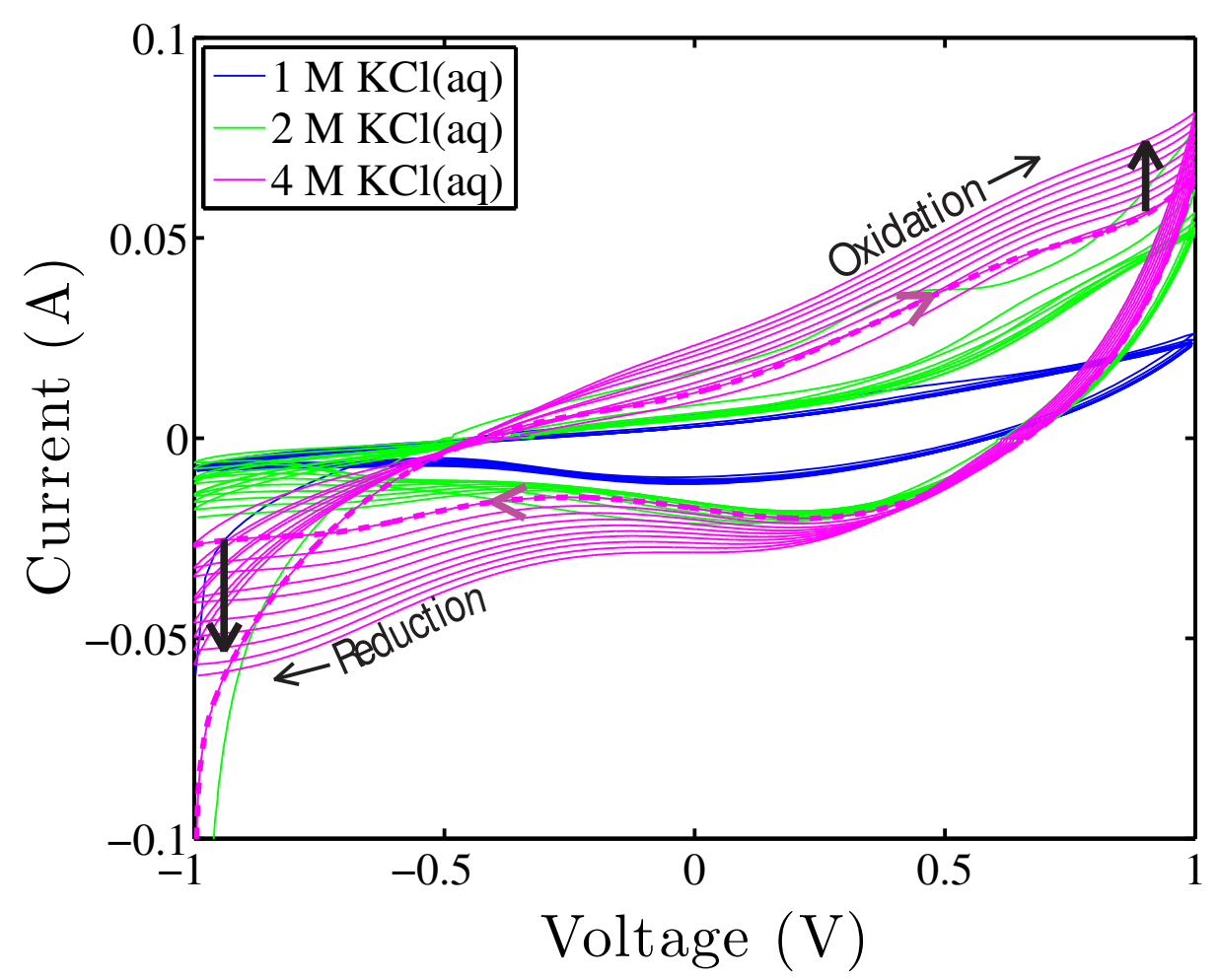

Figure 4.1: Cyclic voltamograms of PPy membranes on stainless steel plate electrodes, dipped in either $1 \mathrm{M}$ (blue curve), $2 \mathrm{M}$ (green) or $4 \mathrm{M} \mathrm{KCl}_{a q}$ (magenta). A voltage is linearly swept from $-1 \mathrm{~V}$ to $+1 \mathrm{~V}$ vs. SCE for 10 cycles at $100 \mathrm{mV} / \mathrm{s}$ rate. The currents obtained from these varying potentials give information on the reaction kinetics between the membrane and $\mathrm{KCl}_{a q}$. As denoted, a positive voltage oxidizes the membrane, while a negative one reduces it. 
Immediately evident from Figure 4.1 is the charging behavior of the membranes becoming more pronounced in the presence of increasing $\mathrm{KCl}_{a q}$ concentrations, as the lines fan out from blue, to green, to magenta (1, 2 and $4 \mathrm{M} \mathrm{KCl,} \mathrm{respectively).} \mathrm{These}$ increasing currents correspond to the membrane's increasing ion uptake from the surrounding solution during redox cycling. As expected, these charges increase with increasing surrounding concentrations of the salt, as more charge capture events occur due to their increased availability. These ions may be up-taken via weak association to charge balance the membrane, or may be involved in ion-polymer binding events.

As the solution concentration increases from $1 \mathrm{M}$ to $4 \mathrm{M} \mathrm{KCl}_{a q}$, so does the hysteresis seen between voltage cycles, with the smallest hysteresis occurring at $1 \mathrm{M} \mathrm{KCl}$ (Figure 4.1, blue curves). The largest hysteresis is seen in $4 \mathrm{M} \mathrm{KCl}_{a q}$ solution (magenta curves), where the initial cycle is depicted by the dotted line, and subsequently fans outward in the direction of the black arrows over each following cycle. In fact, the larger the electrolyte concentration, the larger the hysteresis.

Based on key findings by Akieh and others [154, 155, 155-157], it is known that during PPy oxidation, anions (or in this case $\mathrm{Cl}^{-}$ions) are uptaken into the membrane to neutralize the newly formed cationic centers. Conversely, during membrane reduction $\mathrm{K}^{+}$ions are uptaken to charge neutralize the semi-immobile TOS $^{-}$counterions in the membrane, while chloride ions are expelled. Although $\mathrm{TOS}^{-}$counterions are somewhat mobile within the membrane, only those located at the surface of PPy will be lost to the surrounding electrolyte during long intervals of redox. Therefore, charge compensation via electroneutralization occurs predominantly from $\mathrm{KCl}$ salt exchanges within the membrane during short time periods.

Given this knowledge and the concentration dependent hysteresis seen in Figure 4.1, we hypothesize that this represents a shift in the ion exchange process, due to a more stable ion pair formation between $\mathrm{K}^{+}$and the remaining $\mathrm{TOS}^{-}$counterion within the membrane when the salt is present in such high concentrations. These ions left behind after reduction are known as retention, according to Majumdar et al. [158], 
and may cause creep or fatigue in the membrane over many cycles. These retained ions may even form stable bonds with the cationic centers on the polymer chains, reducing the redox capability of the membrane by interfering with the $\pi$-centers* ${ }^{*}$ It is recommended, therefore, that despite the appeal to increase flux rates with larger concentration gradients of $\mathrm{KCl}$ across the membrane, to stay below levels which induce large hysteresis in the CVs. This information dictated that flux experiments should be performed in no more than $1 \mathrm{M} \mathrm{KCl}_{a q}$ feed solutions.

\subsubsection{Scanning Electron Microscopy}

Scanning electron microscopy (SEM) images of the surface and cross-sectional view of PPy after deposition (see Section 3.2.2 for details) are shown below in Figure 4.2. The surface morphology of PPy (Figure 4.2, left) displays distinct cauliflowerlike nodules typical of these membranes. The cross-sectional view of PPy (Figure 4.2 , center) reveals the membrane thickness, which was determined as a function of deposition duration and charge (see Section 4.1.3). The image on the right in Figure 4.2 below shows the morphology of the back side of PPy, which grew in contact with the stainless steel working electrode, leaving a smooth finish with details of the working electrode imprinted onto its surface. These results are typical of the literature [159].

\footnotetext{
${ }^{*} \pi$-centers are regions of delocalized and overlapping electron orbitals situated between the carbon atoms of the pyrrole aromatic ring. The overlapping electron orbitals and electron sharing occurring at these centers allow for the easy travel of electrons (and hence high electronic conductivity) down the PPy polymer chains. $\pi$-bonds are therefore the central areas which make polypyrrole a conductive material.
} 

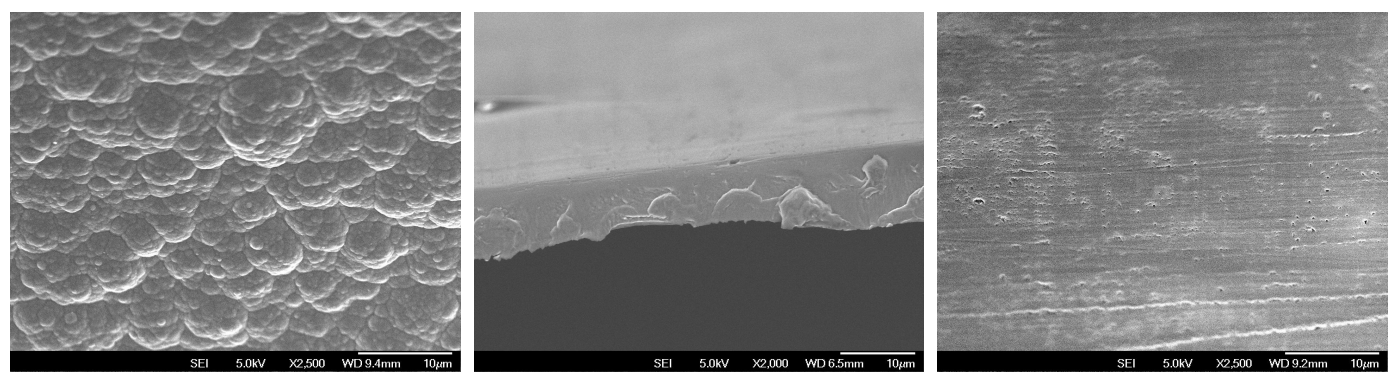

Figure 4.2: Scanning electron microscopy images of PPy. Left: The surface morphology of PPy is shown at 2,500X magnification, with visible cauliflower-like nodules. Center: A cross-section of PPy is shown at 2,000X magnification. The flat top half of the membrane corresponds to the electrode side of the PPy, and the softly undulating surface to the bottom is the membrane's surface, same as shown to the left. Right: The back side of PPy, which was in contact with the working electrode during deposition, is also shown at 2,500X magnification. The smooth morphology here is due to the mirror finish stainless steel electrode.

Evident from these SEM images is a lack of a pore structure at the magnifications shown. In fact, higher magnifications still show no observable pores in PPy, implying that the membrane's structure is very finely meshed and ion transfer must occur through nanometer size pores.

Finally, the asymmetry of PPy from one surface to the other and the unknown effects these morphologies may have on the flux of ions through the membrane underscores the importance of consistent directionality when placing the PPy membrane in the flux chamber setup. As such, the smooth side of PPy (Figure 4.2, right) was always placed facing the feed solution $\left(1 \mathrm{M} \mathrm{KCl}_{a q}\right)$ for reproducibility during these experiments.

\subsubsection{Thickness Determination and Prediction}

Membrane thickness values were determined via SEM for a range of deposition times and current densities (Figure 4.3, red and black circles). These data help to understand the effects of deposition parameters on the membrane properties, and provide the necessary knowledge to adjust the membrane characteristics in order to satisfy ion gate design criteria. These data were also used to test the validity of a membrane thickness estimate (see Equation 4.1) set forth by Farrington et al. [148]. 
Indeed, having a reliable predictive model for the thickness of PPy would replace the need for SEM on membranes.

The empirical values obtained via SEM were therefore compared to the theoretical predictions derived from Farrington's model (Figure 4.3, $\bigcirc$ and $\times$ marks, respectively). All variables used in the Farrington model (Equation 4.1) were as described in Section 3.2.1, including the assumptions of a fixed membrane density $\rho=1.1 \mathrm{~g} / \mathrm{cm}^{3}$ and a fixed number of pyrrole units per anion $y=3$.

$$
d=\frac{Q}{n \cdot F \cdot A \cdot \rho}\left[M_{1}+\frac{M_{2}}{y}\right],
$$

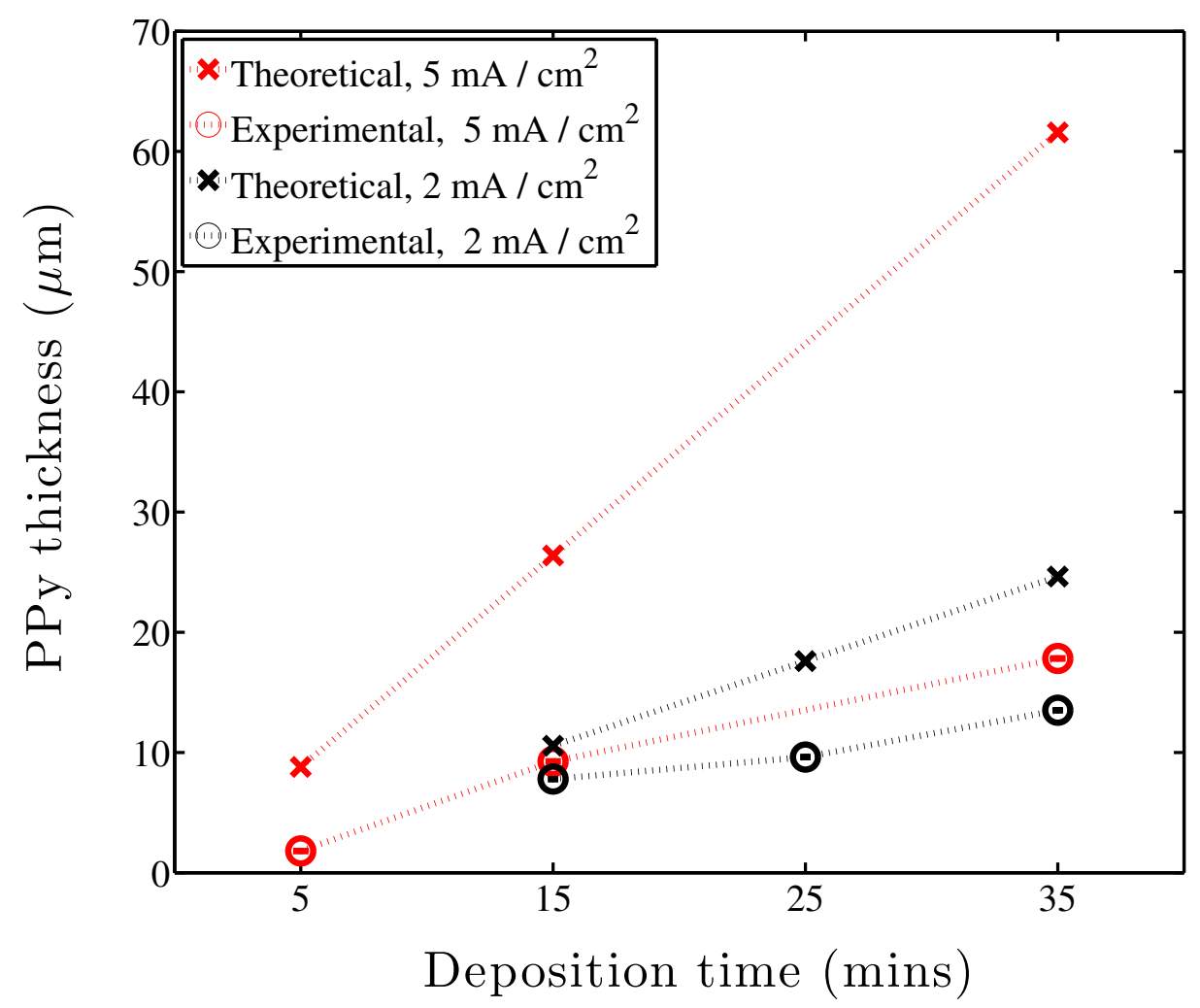

FiguRE 4.3: PPy thickness measurements via SEM ( $\bigcirc$ marks) are compared to theoretical predictions $(X$ marks) based on work by Farrington et al. Red represents PPy membranes produced at $5 \mathrm{~mA} / \mathrm{cm}^{2}$, and black represents those at $2 \mathrm{~mA} / \mathrm{cm}^{2}$. The original assumption that PPy density is approximately $1.1 \mathrm{~g} / \mathrm{cm}^{3}$ is maintained in Equation 4.1. The theoretical model overpredicts the PPy thicknesses for both deposition current densities.

As seen in Figure 4.3, Farrington's model ( $\boldsymbol{X}$ marks) overestimates the actual 
membrane thickness ( $\bigcirc$ marks) for both deposition conditions, i.e. at $5 \mathrm{~mA} / \mathrm{cm}^{2}$ and $2 \mathrm{~mA} / \mathrm{cm}^{2}$. A likely explanation for this discrepancy is the model's assumption of a fixed PPy density at $1.1 \mathrm{~g} / \mathrm{cm}^{3}$. Many groups have assumed a rounded value for density, including Akieh et al., who approximated an even lower density of $1 \mathrm{~g} / \mathrm{cm}^{3}$ [160]. However, according to other research on PPy, the lower the current density deposition, the higher the density of PPy membranes [158, 161]. Since densities clearly cannot be approximated as a constant, the fixed density assumption for PPy is an oversimplification in Farrington's model and may at least partially account for the predicted thickness values being so far off (See Figure 4.7: where red and blue bars represent the percent error between empirical and theoretical values). It was hypothesized that the density approximation of $1.1 \mathrm{~g} / \mathrm{cm}^{3}$ was likely the main cause for error.

Membranes were therefore sent for testing via pycnometer at the Micromeritics Analytical Services laboratories to determine their absolute densities. The results, as seen in Figure 4.4, show that PPy density is indeed poorly represented by the 1.1 $\mathrm{g} / \mathrm{cm}^{3}$ assumption. In fact, all of the measured densities are well above $1.1 \mathrm{~g} / \mathrm{cm}^{3}$ and show a nonlinear response to deposition times and current density. It is interesting to note that membrane density drops significantly with deposition time. This may be a result of a decreasing charge density available at the end of extending polymer branches, making polymerization events there more infrequent as they extend away from the electrode. It is also apparent that higher charge densities lead to lower density membranes, as seen when comparing equal deposition lengths of time. More data would be needed, however, to determine exactly why density varies with charge density and deposition time. 


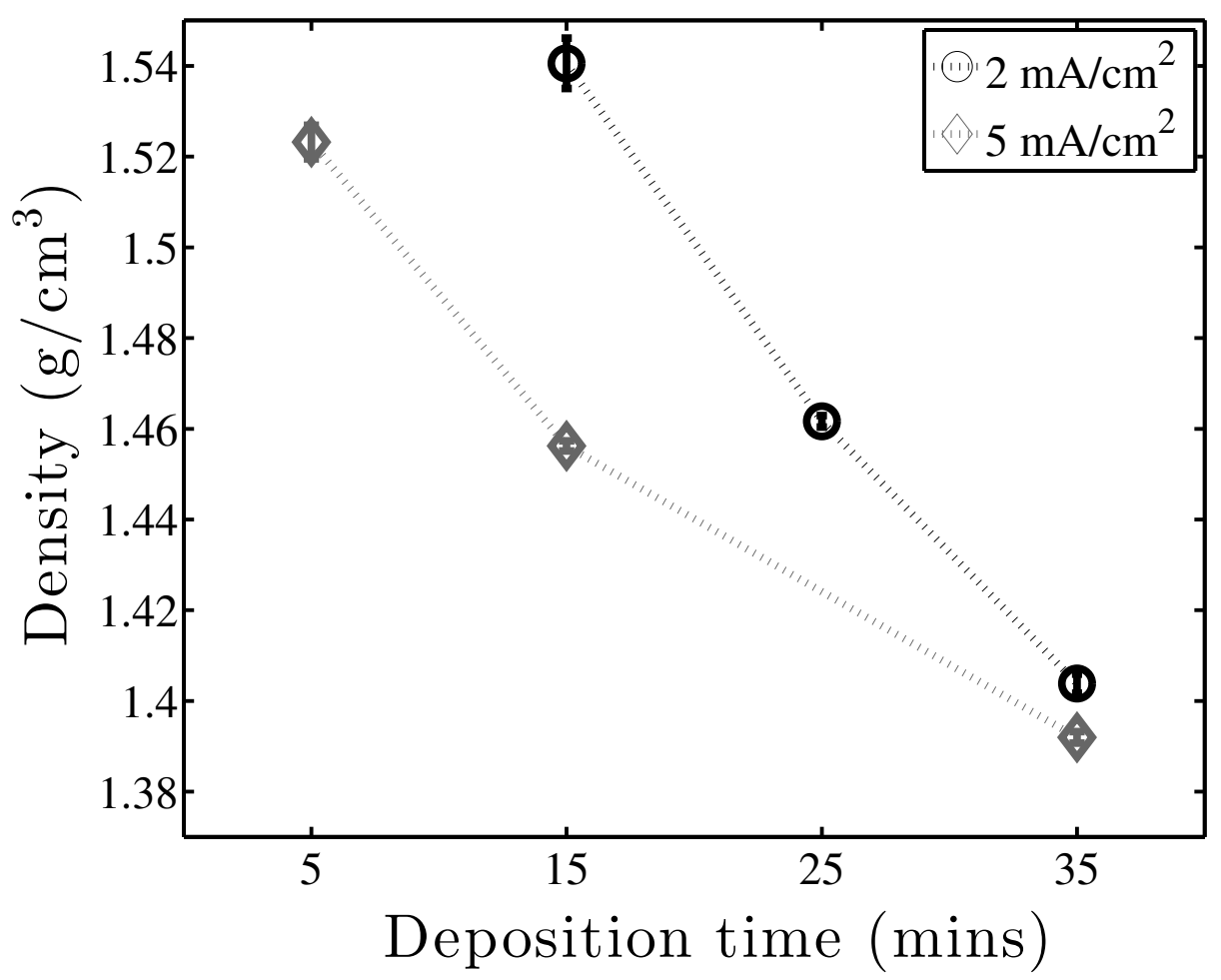

FiguRE 4.4: Experimentally determined absolute density values are shown as a function of deposition time in PPy membranes produced at either $2 \mathrm{~mA} / \mathrm{cm}^{2}(\bigcirc)$ or $5 \mathrm{~mA} / \mathrm{cm}^{2}(\diamond)$. A gas density method using a pycnometer was performed on $\mathrm{n}=10$ membranes at each condition at the Micromeritics Analytical Services laboratory.

To correctly predict membrane thickness, this nonlinearity must be considered in the Farrington model. These experimentally obtained density values for PPy were therefore used for a new calculation on the predicted thickness of the membranes, as shown in Figure 4.5, on the right (which is placed next to the original thickness predictions from Figure 4.3, for ease of comparison). It is clear that the experimental membrane density values offer a significant improvement in thickness prediction for the Farrington model. 

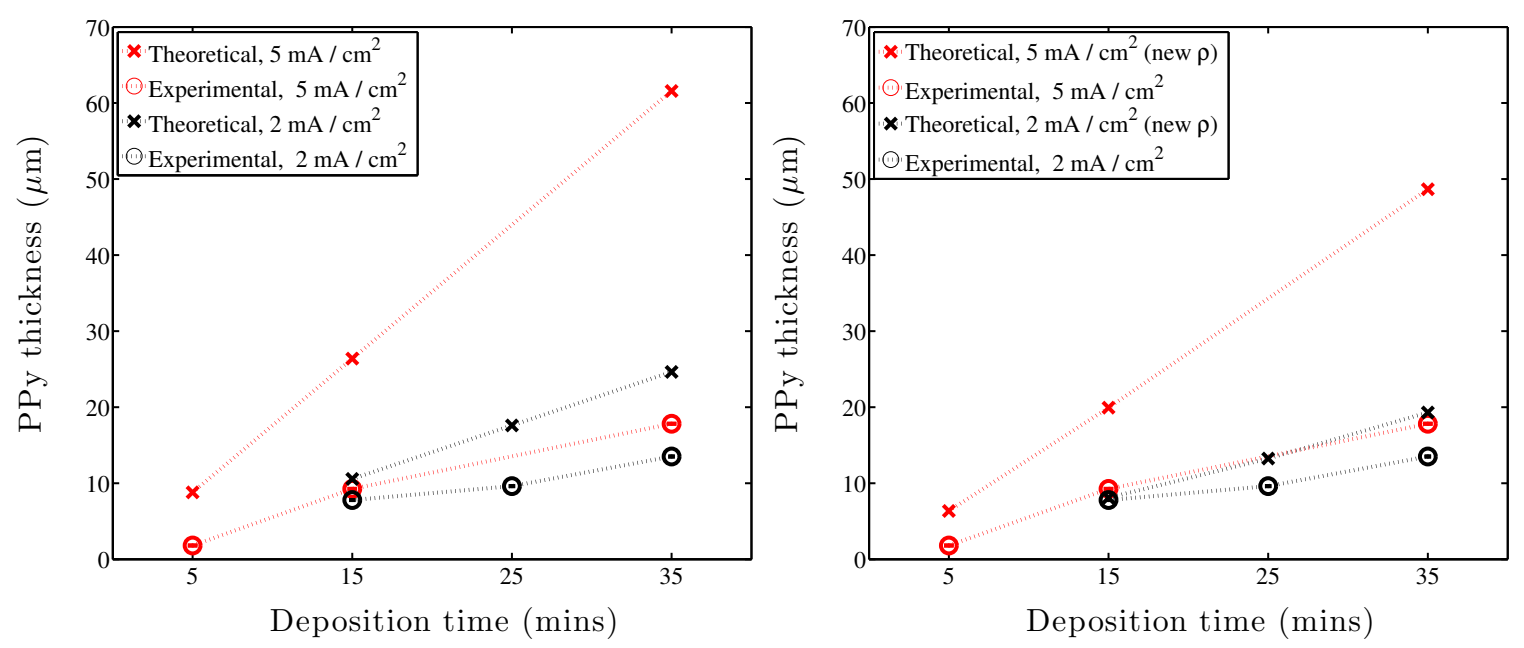

Figure 4.5: Left: The original assumption that PPy density is approximately $1.1 \mathrm{~g} / \mathrm{cm}^{3}$ is maintained in Equation 4.2, and the theoretical model significantly over-predicts the PPy thickness. Right: The densities used to calculate thickness via the Farrington model are experimentally derived, bringing the Farrington model closer to the experimental thickness values obtained.

It was hypothesized that in addition to variable densities, the value of $y$ (which is the number of pyrrole units per anion) should also be considered variable, instead of given an arbitrarily fixed value [148]. In fact, since the density of membranes decreases over time during deposition, it is likely the structure of the PPy itself changes dramatically as strands extend further away from the working electrode and experience lower charge densities. It is hypothesized that this could alter the ratio of pyrrole units to anions considerably, justifying increasing values of $y$ as a function of deposition time in the Farrington model. The values of $y$ were therefore iteratively increased at each time point until they minimized the percent error between the model and the experimental thickness values.

Although this approach will require further consideration as it remains speculative, the new $y$ values and experimentally determined densities, combined, allowed for significantly improved predictions of the membrane thickness, as seen in Figure 4.6, on the right. The percent error before and after these corrections also reflect the model's improvement, as seen in Figure 4.7 (colored vs. black bars). These data demonstrate the precision of the modified Farrington model at predicting membrane thickness values at $2 \mathrm{~mA} / \mathrm{cm}^{2}$ current density. However, the model appears to remain 
inadequate at predicting the thicknesses produced at $5 \mathrm{~mA} / \mathrm{cm}^{2}$, suggesting a more complex phenomenon occurring at the higher current densities, which may not be accounted for in the model.
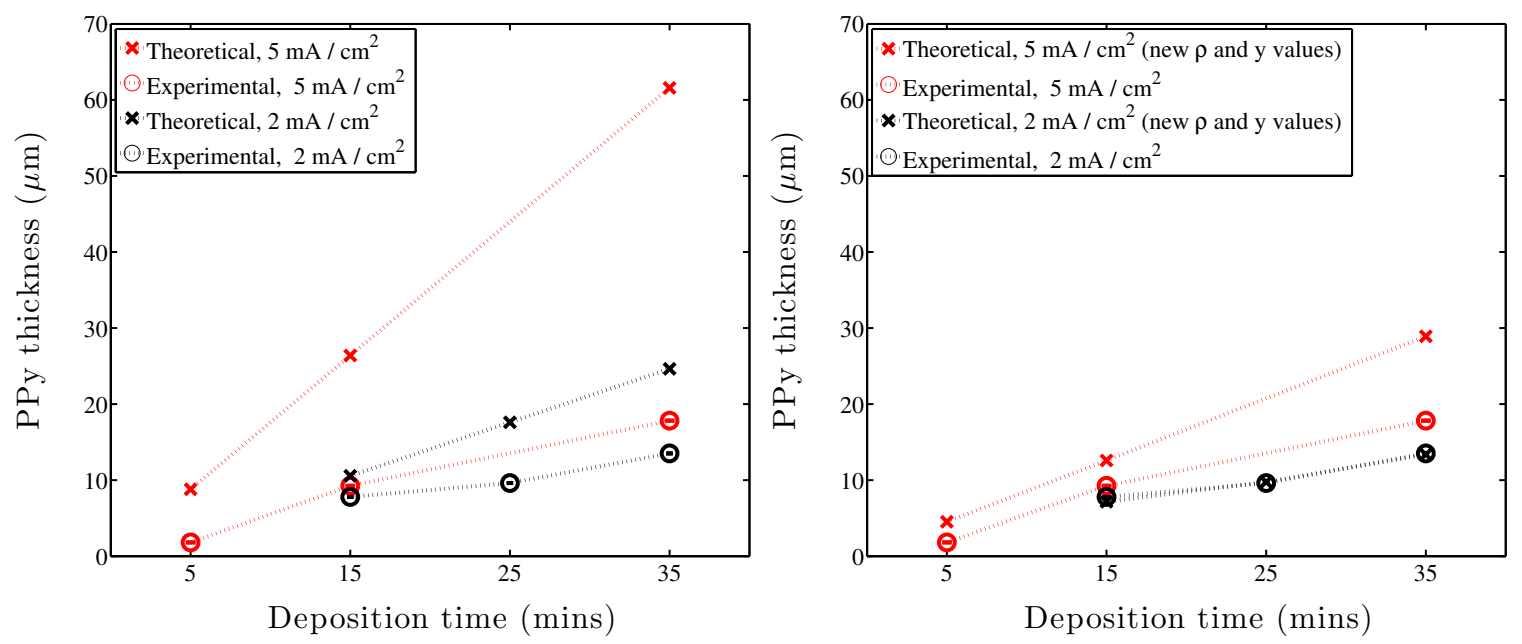

FiguRE 4.6: PPy thickness measurements via SEM ( $\bigcirc$ marks) are compared to theoretical predictions $(X$ marks) based on work by Farrington et al. Red represents PPy membranes produced at $5 \mathrm{~mA} / \mathrm{cm}^{2}$, and black represents those at $2 \mathrm{~mA} / \mathrm{cm}^{2}$. Left: The original assumption that PPy density is approximately $1.1 \mathrm{~g} / \mathrm{cm}^{3}$ is maintained in Equation 4.2 , and the theoretical model fails to accurately predict the PPy thickness. Right: The densities used to calculate thickness via the Farrington model are experimentally derived, and y values are a non-linear function of charge density. Comparing to SEM thickness data, the model now more accurately predicts the PPy membrane thickness, especially in the case of membranes produced at $2 \mathrm{~mA} / \mathrm{cm}^{2}$ (black $X$ and $\bigcirc$ 's overlap nearly perfectly). 


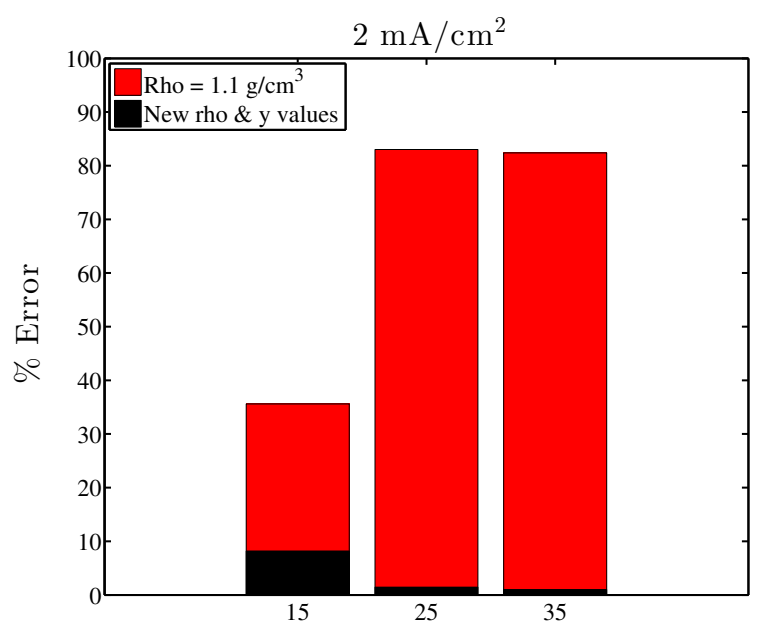

Deposition time (mins)

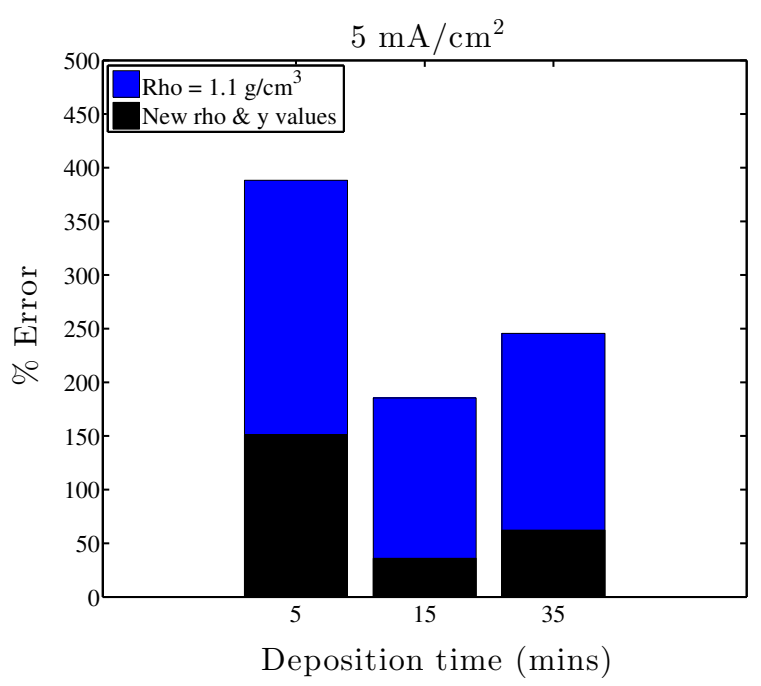

FiguRE 4.7: Graphs representing percent error between Farrington's model prediction and measured membrane thickness values are shown, before and after model assumption changes. Left: Red bars represent percent error when using the original model assumptions of $\rho=1.1 \mathrm{~g} / \mathrm{cm}^{3}$ and $\mathrm{y}=3$ for membranes deposited at $2 \mathrm{~mA} / \mathrm{cm}^{2}$. Superimposed black bars represent the percent error after using experimentally determined $\rho$ values and adjusted y values. Right: Blue and black bars represent the same as red and black bars, however this time in membranes produced at $5 \mathrm{~mA} / \mathrm{cm}^{2}$.

Regardless, these data do validate the use of the Farrington model for predicting membrane thickness, especially in the case of lower current density, while hinting at PPy's structural properties varying significantly in response to deposition conditions. Indeed, it is useful to possess a precise model for the prediction of membrane thickness (and avoid the need for SEM) when seeking to modify deposition parameters in order to adjust membrane properties.

Suggested revisions to Farrington's model would account for density $(\rho)$ and number of pyrrole units per anion $(y)$ as functions of the charge density seen at the membrane during deposition (see Equation 4.2). The exact nature of the relationship between density, $y$ and charge density is left for future consideration.

$$
d=\frac{Q}{n \cdot F \cdot A \cdot \rho(f(Q))}\left[M_{1}+\frac{M_{2}}{y(f(Q))}\right]
$$




\subsubsection{Comparable Membranes from Different Deposition Schemes}

Now that density has been addressed in terms of thickness modeling, it is of interest to revisit membrane density and thickness directly. Two membranes are of particular interest in the figures above, which despite being produced at different current densities (2 and $5 \mathrm{~mA} / \mathrm{cm}^{2}$ ) and different deposition durations (25 and 15 minutes), result in comparable membrane thicknesses (as seen in Figure 4.8 below). These are the $5 \mathrm{~mA} / \mathrm{cm}^{2}, 15$ minute deposition membrane $\left(\mathrm{PPy}_{a}\right)$, and the $2 \mathrm{~mA} / \mathrm{cm}^{2}, 25$ minute membrane $\left(\mathrm{PPy}_{b}\right)$, which have 9.3 and $9.6 \mu \mathrm{m}$ thick membranes, respectively.

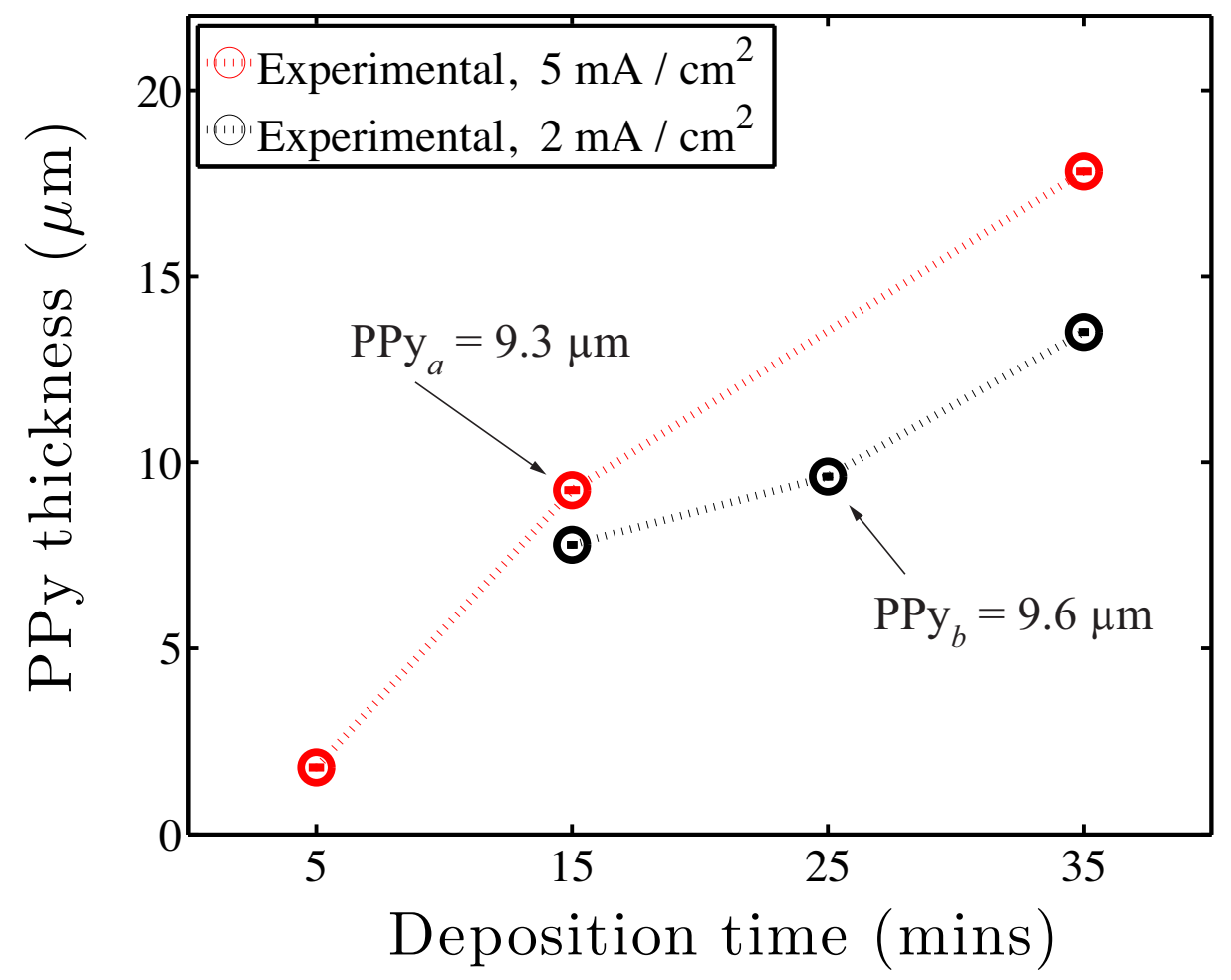

FIgURE 4.8: PPy thickness measurements via SEM are shown for membranes produced at 5 $\mathrm{mA} / \mathrm{cm}^{2}$ (red), and $2 \mathrm{~mA} / \mathrm{cm}^{2}$ (black). Two membranes resulted in comparable thickness measurements despite being produced under different conditions: $\mathrm{PPy}_{a}$ and $\mathrm{PPy}_{b}$, as marked.

Although they have similar thicknesses, according to Muramatsu et al., they likely exhibit significant structural differences, as evidenced by the changing viscoelastic material properties their group observed between identical PPy membranes produced at $0.5 \mathrm{~mA} / \mathrm{cm}^{2}$ and $6 \mathrm{~mA} / \mathrm{cm}^{2}$ [157]. The thickness difference between $\mathrm{PPy}_{a}$ and 
$\mathrm{PPy}_{b}$ is small enough that it is of interest to compare them, but we cannot neglect it either, as flux rates will be influenced by small differences in travel distance through the material. Despite this, it is useful to find and compare two membranes of similar thickness, synthesized under different current densities, to test how much of an effect this will have on their structural properties, and ultimately their ion gating ability.

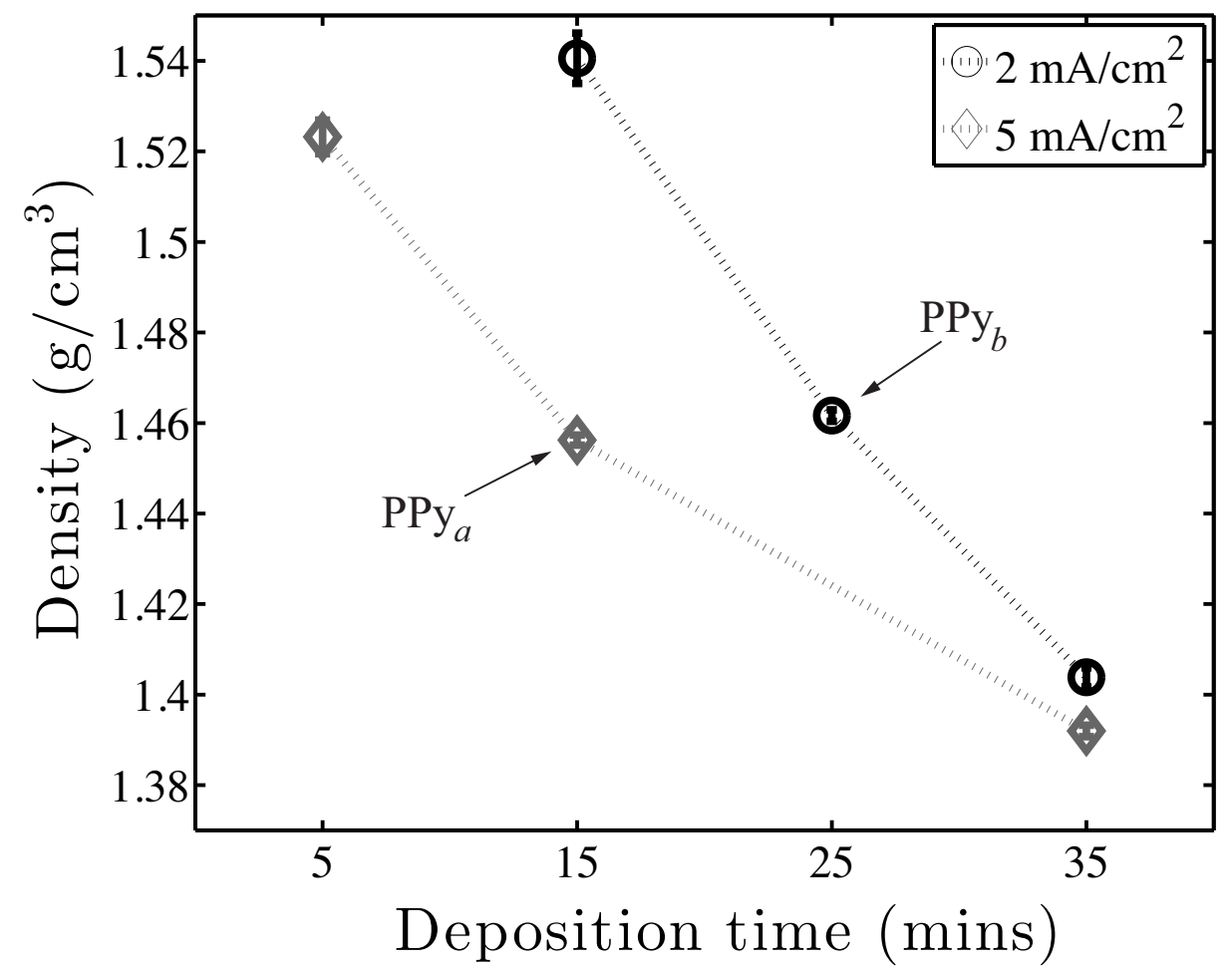

Figure 4.9: Experimentally determined absolute density values are shown as a function of deposition length in PPy membranes produced at either $2 \mathrm{~mA} / \mathrm{cm}^{2}(\bigcirc)$ or $5 \mathrm{~mA} / \mathrm{cm}^{2}(\diamond)$. A gas density method using a pycnometer was performed on $\mathrm{n}=10$ membranes at each condition at the Micromeritics Analytical Services laboratory.

In fact, looking at the results from Figure 4.9 and comparing the points for $\mathrm{PPy}_{a}$ and $\mathrm{PPy}_{b}$, at first glance their densities look nearly identical. However, upon closer inspection and by one-way ANOVA, the two densities are determined to be significantly different $(\mathrm{p}=0.005)$, with the slightly thicker membrane having a more dense core. One would expect, intuitively, that a thicker and denser membrane would provide increased resistance to ion flow, resulting in smaller flux rates through $\mathrm{PPy}_{b}$, if flux were simply a function of passive diffusion when the membrane is in the open 
state. However, this is not the behavior we observe in PPy membranes, as will be presented in Section 4.3, which hints at an active component of ionic cycling through PPy. This will be discussed further as we compare the flux rates between these two specific membranes.

\section{$4.2 \quad$ Hydrogel}

Hydrogels have two key properties that make them very similar to, and compatible with, biological tissues: a high water content and rubber-like mechanical properties. They display both macroscale solid mechanical stability, while retaining liquid phase diffusive behavior within their pore structure. In these studies, the water content for hydrogels is reported, and the concentration of potassium chloride ions loaded in acrylamide hydrogels is successfully characterized via electrochemical impedance spectroscopy. These data give insight on the liquid phase behaviors of these materials and the ions that permeate through them, findings which will be leveraged for the development of the artificial cell.

\subsubsection{Water Content Determination}

Polymer crosslinking is directly related to water content and material properties of hydrogels, such as Young's Modulus and solute diffusivity. The crosslinking density, defined as the number of polymer chain crosslinks divided by the number of monomers, can be used to fine-tune water and solute diffusion within the polymer matrix, qualities very relevant in application to the gel-cell. A correlative value to cross linking density in hydrogels is the equilibrium water content (EWC), which is determined by the weight difference between fully hydrated hydrogel and it's dehydrated equivalent [95, 162], as shown in Equation 4.3:

$$
\operatorname{EWC}(\%)=\frac{W_{\text {wet }}-W_{\text {dry }}}{W_{\text {wet }}} \times 100
$$


The EWC ratios obtained for $2 \mathrm{~nm}$ pore size (4.8\% crosslinker) hydrogels (first soaked in various concentrations of $\mathrm{KCl}_{a q}$ for over 48 hours, then dried for several days) are shown in Figure 4.10 below. The descending EWC ratio values indicate $\mathrm{KCl}$ salts were left behind in the hydrogel structures as they dried. This provided first line evidence that $\mathrm{KCl}_{a q}$ was being absorbed into the hydrogel pore structure, which is expected since cations are known to be absorbed non-reversibly in hydrogels. In fact, this ion-structure capture quality in hydrogels has been harnessed to remove metallic ions such as $\mathrm{Cu}^{2+}$ impurities from wastewater [163]. From these data, however, it was impossible to determine the molarity of $\mathrm{KCl}_{a q}$ absorbed into the polymer, and therefore a more precise measurement tool was needed.

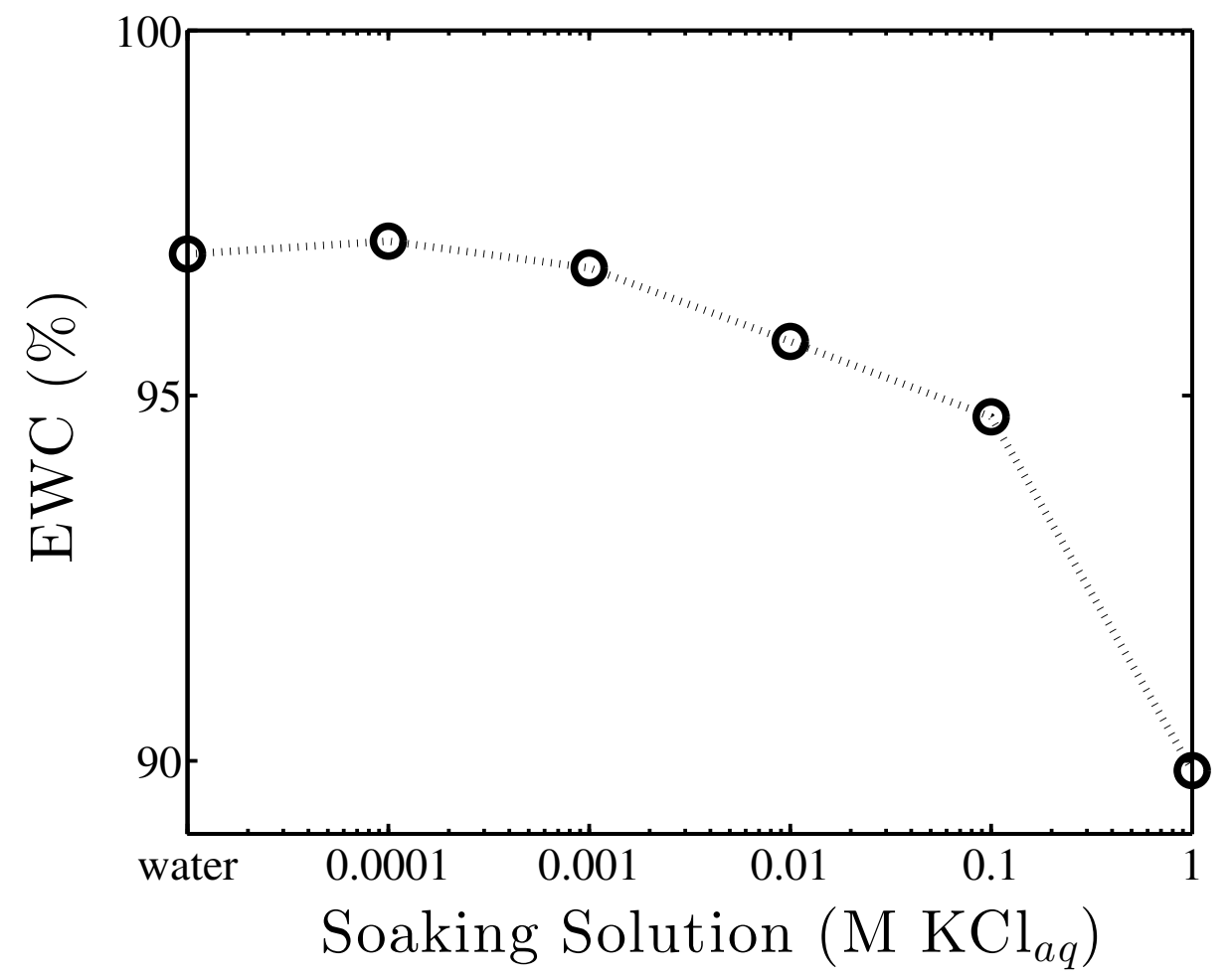

FIgURE 4.10: Equilibrium water content of $4.8 \%$ crosslinker acrylamide hydrogels, pre-soaked in varying concentrations of $\mathrm{KCl}_{a q}$, from $\mathrm{diH}_{2} \mathrm{O}$ to $1 \mathrm{M}$. 


\subsubsection{Electrochemical Impedance Spectroscopy}

The ability to detect the presence of potassium ions and their concentration in precise locations within hydrogels is critical for the development of the artificial cell and to determine ion flux rates achieved through the artificial membrane. Electrochemical impedance spectroscopy (EIS) was hypothesized as a potential measurement technique which could do just that: detect small changes in material conductivity at the location of it's probes in the hydrogel as a result of changing $\mathrm{KCl}_{a q}$ concentrations. This method was first tested in solution to determine its baseline sensitivity for potassium chloride ions in $\mathrm{diH}_{2} \mathrm{O}$. Once successfully calibrated there, the method was used in hydrogel samples soaked in the same $\mathrm{KCl}_{a q}$ concentration range. The sensitivity of EIS within hydrogel was found to be only slightly lower than in solution, and nearly unaffected in the pore sizes tested (Figure 4.11). As a result, the in-house parallel plate EIS system was used to detect the presence of potassium chloride in the flux experiments, both in solution and hydrogel.

Calibration curves in aqueous potassium chloride solutions ranging from $10^{-5} \mathrm{M}$ $\mathrm{KCl}_{a q}$ to $1 \mathrm{M} \mathrm{KCl}_{a q}$ were performed. A log-log linear response is observed by the EIS probe between $10^{-4} \mathrm{M}$ and $1 \mathrm{M} \mathrm{KCl}_{a q}$, placing the detection limit of the probe in solution at $10^{-4} \mathrm{M}$ (Figure 4.11, magenta curve). 


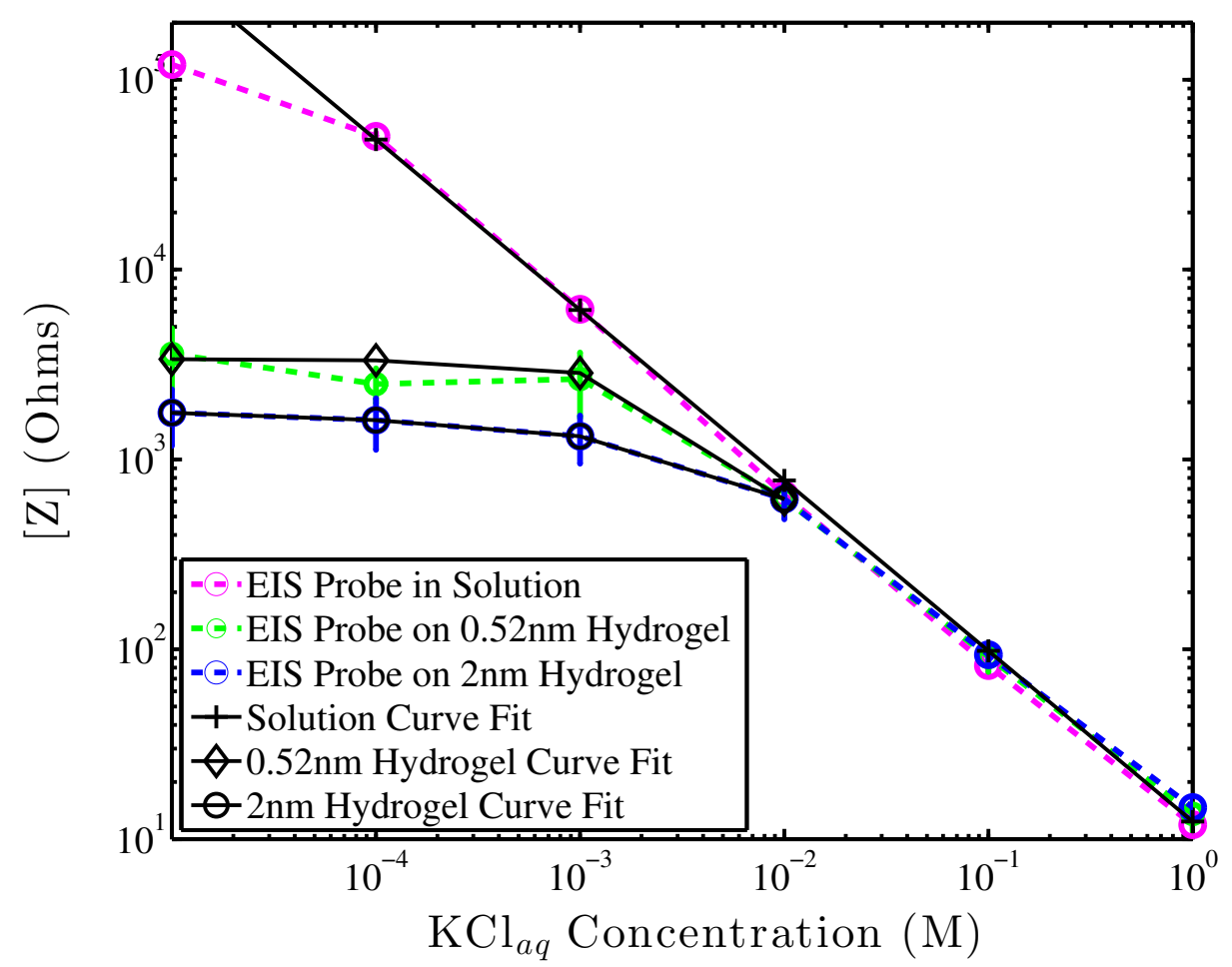

FIGURE 4.11: EIS probe calibration curves are shown here in solution (magenta) and two different pore size hydrogels $(0.52 \mathrm{~nm}$ and $2 \mathrm{~nm}$; green and blue, respectively), with respective curve fit overlays (black lines). The impedance magnitude response of the probe $(|Z|(\mathrm{Ohms}))$ is shown versus a range of $\mathrm{KCl}_{a q}$ concentrations $(\mathrm{n}=3)$. Hydrogels were soaked in $\mathrm{KCl}_{a q}$ solutions for 48 hours before testing. Best fits to the data are also shown for EIS in solution $(+), 0.52 \mathrm{~nm}$ hydrogels $(\diamond)$ and $2 \mathrm{~nm}$ hydrogels $(\bigcirc)$. The EIS in solution was best represented by a dipole fit, where $\mathrm{y}=$ $12.392 \mathrm{x}^{-449 / 500}$. Since EIS in both hydrogels followed the same trend down to $10^{-2} \mathrm{M} \mathrm{KCl}_{a q}$, a piecewise fit was applied to these data. The dipole fit extended to $10^{-2} \mathrm{M}$, below which an exponential curve was fit to both hydrogels. For the $0.52 \mathrm{~nm}$ and $2 \mathrm{~nm}$ hydrogels, respectively, $\mathrm{y}=$ $\mathrm{a} \cdot \exp ^{b \cdot x}$ and $\mathrm{y}=\mathrm{c} \cdot \exp ^{d \cdot x}+\mathrm{e} \cdot \exp ^{f \cdot x}($ where $\mathrm{a}=3379, \mathrm{~b}=-170, \mathrm{c}=1442, \mathrm{~d}=-84, \mathrm{e}=338$, and $\mathrm{f}=-6174)$.

The EIS probe was also calibrated in hydrogel of two different pore sizes; $2 \mathrm{~nm}$ and $0.52 \mathrm{~nm}$ (Figure 4.11 - blue and green curves, respectively). It is apparent from these curves that in addition to the EIS response in both hydrogels being linear above $10^{-2} \mathrm{M} \mathrm{KCl}_{a q}$, that all three curves coincide in this region. This means the calibration produced in solution may be used interchangeably for hydrogels in this higher concentration domain. These curves also show a detection limit for potassium chloride in hydrogel of $10^{-3} \mathrm{M}$ for both pore sizes. The sensitivity to $\mathrm{KCl}_{a q}$ is reduced between $10^{-2}$ and $10^{-3} \mathrm{M}$, likely due to a predominance of the hydrogel polymer 
structure on the impedance reading. The lower detection limit found in hydrogels is still quite suitable for use in the studies on gel-cells, as the concentration of $\mathrm{KCl}_{a q}$ found in hydrogel will be on the order of 1 molar. The higher detection limit, however, will reduce the probe's sensitivity to small ion fluxes across the membrane at short time periods.

As seen in Figure 4.11, the dipole curve fit for EIS in solutions was excellent when applied between 1 and $0.0001 \mathrm{M} \mathrm{KCl}_{a q}$, with $\mathrm{R}^{2}=0.999$. In hydrogels, however, a deviation from the solution response started around $0.01 \mathrm{M} \mathrm{KCl}_{a q}$, requiring a piecewise calibration for hydrogels. The piecewise curve fit consisted of the solution's fit until the break off concentration $(0.01 \mathrm{M})$, followed by exponential curve fits for both $2 \mathrm{~nm}$ and $0.52 \mathrm{~nm}$ hydrogels, with $\mathrm{R}^{2}=1$ and 0.9947 , respectively. These calibration curves and their curve fit relationships were used to determine the precise ion concentrations in liquid and hydrogel environments in order to determine ionic flux rates across PPy membranes in both of these environments.

\subsection{Flux Experiments}

Studies were performed to determine optimal membrane preparation and cycling conditions for maximum ion flux through the membrane. First, time-resolved experiments quantified potassium chloride flux rates immediately following membrane opening, and helped select optimal membrane cycling parameters for peak flux. Sec-

ondly, time-lapse experiments highlighted the importance of membrane density and thickness effects on flux rates. Finally, flux experiments were performed for the first time on a PPy/hydrogel system using the optimal membrane conditions.

\subsubsection{Time-Resolved Concentration Readings}

The standard protocol used to determine flux rates through PPy involves removing samples from the receiving solution at discrete time intervals, and testing these for 
the presence of ions [154]. Physically removing samples from the experimental setup in small aliquots (sometimes up to $5 \%$ of the overall volume of solution) presents many obvious drawbacks. Most importantly, however, discretized sampling of the receiving solution at large time intervals makes it impossible to determine the short term kinetics of ion flux through the membrane.

To obtain more detailed information regarding the short-term response of the ion gate in solution, a time-resolved recording of the concentration profiles of $\mathrm{KCl}_{a q}$ as it passes through the membrane was produced. This was made possible, as described in the methods chapter, in Section 3.5.2, by connecting two potentiostats in parallel for simultaneous reading on the EIS probe and voltage application at the membrane.

An example signal response from EIS is seen in Figure 4.12, which shows the concentration of $\mathrm{KCl}_{a q}$ in the receiving solution over time. Signal noise from the probe was filtered, as shown, using a Loess filter, which is a quadratic local regression fitting tool available in the Matlab software package. An interpolation of the data was also performed as shown. 


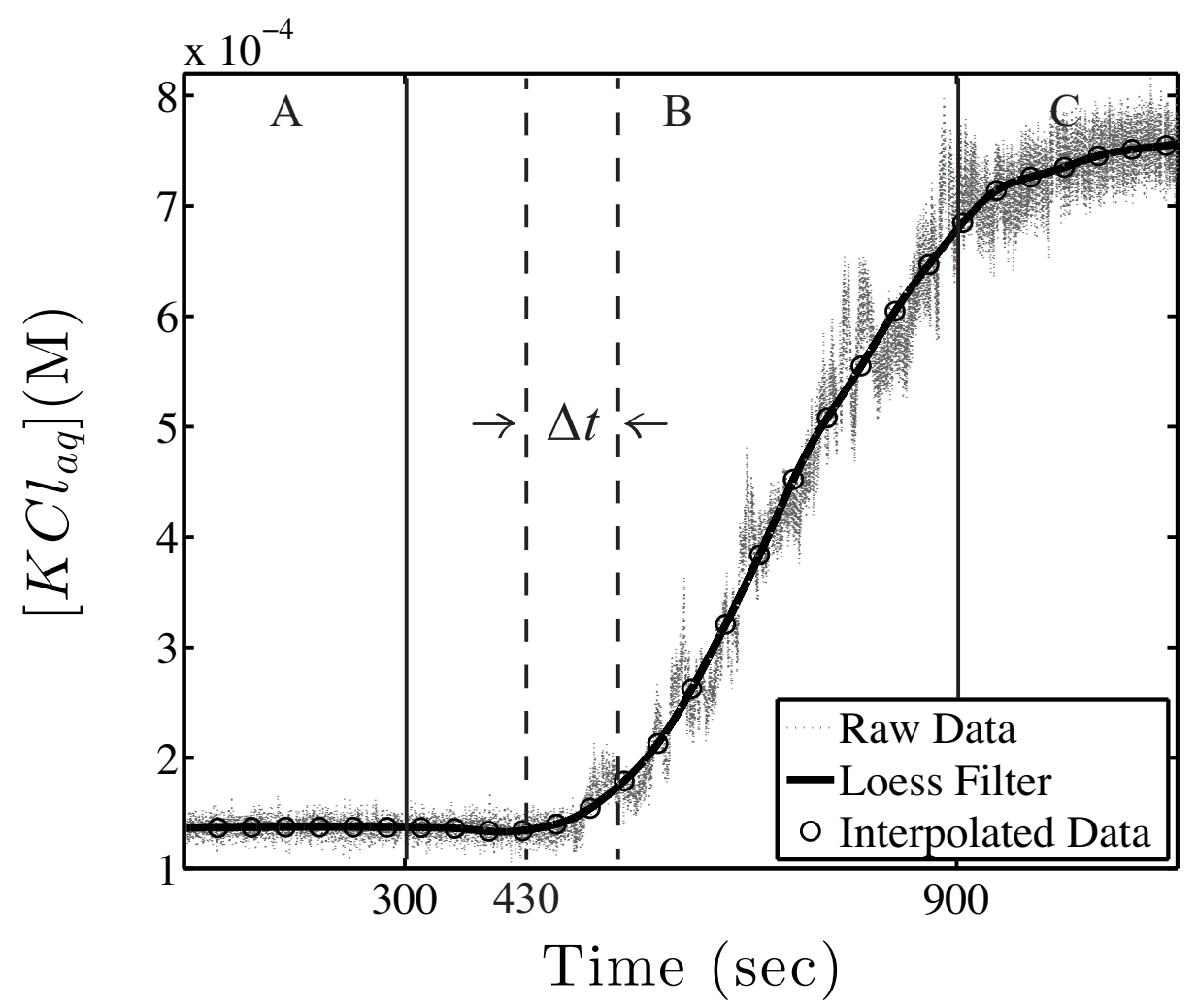

FIgURE 4.12: Example time-resolved concentration profile for $\mathrm{KCl}_{a q}$ through a $\mathrm{PPy} / \mathrm{TOS}^{-}$membrane, obtained via EIS probe and filtered. A: Membrane is at rest for 5 minutes. B: A cycling potential $( \pm 1 \mathrm{~V})$ is applied in 50 second pulse widths at the membrane for 10 minutes. A delayed ionic passage is observed here (130 seconds), which is in excess of a full uptake-and-release cycle on the membrane. The ionic flux rate immediately following this was calculated for $\Delta t=100$ seconds. C: The membrane is closed via the application of $-1 \mathrm{~V}$ vs. SCE. Solid line $(-)$ represents the raw data captured using a VersaSTAT 3 potentiostat by Princeton Applied Research. The sampling rate of the data is one point / second. To reduce signal noise, a Loess filter (local regression using a quadratic fit) was applied to the data (-), followed by an interpolation to reduce the number of data points for ease of analysis $(\bigcirc)$.

To the best of our knowledge, a real-time recording of in situ ion concentrations as they flow through the membrane has never been done before. The information gathered from these real-time readings provided insights into the short-term kinetics of ion release during redox cycling of $\mathrm{PPy}_{\mathrm{TOS}}{ }^{-}$membranes, as demonstrated in the following sections and further considered in the Discussion (Chapter 5).

Immediately apparent in Figure 4.12, is a delay of 130 seconds before the detection of ionic passage, following the application of a cycling voltage to open the membrane. This time represents the membrane "loading time" over a full $\pm 1 \mathrm{~V}$ cycle 
(100 seconds), in addition to some time where ions are being released in the receiving solution, but remain within the noise of the EIS probe reading. Membrane loading time will be explained in more detail in the Discussion.

Molar flux rate of $\mathrm{KCl}_{a q}$ through the membrane for the 100 seconds immediately following detectable ion concentrations, as depicted by $\Delta t$ in Figure 4.12 , is calculated to be $2.36 \times 10^{-8}$ moles $/ \mathrm{cm}^{2} \cdot s$ (using Equation 4.4). At the peak of ion transport, flux rates reach $1.03 \times 10^{-7}$ moles $/ \mathrm{cm}^{2} \cdot s$, and over the duration of the entire 10 minutes of potential cycling, an average flux rate of $5.47 \times 10^{-8}$ moles $/ \mathrm{cm}^{2} \cdot s$ is calculated. It should be noted, however, that this calculated average slightly underestimates the actual flux for $\mathrm{KCl}_{a q}$ through the membrane of $6.61 \times 10^{-8}$ moles $/ \mathrm{cm}^{2} \cdot s$, which was averaged over 500 seconds instead of 600 seconds to account for the ionic transport delay caused by membrane loading. The calculations for all molar fluxes shown from here on, therefore, were corrected by simply reducing the time span used to calculate flux rates by the "membrane loading" time, or 100 seconds, to represent a more accurate estimate of the flux, in the following equation:

$$
J_{i}=\frac{\triangle \mathrm{mol}_{i}}{A_{s} \triangle t},
$$

where $\triangle \mathrm{mol}_{i}$ is the molar influx to the receiving solution of ion $i, \mathrm{~A}_{s}$ is the surface area of the PPy membrane and $\triangle \mathrm{t}$ is the duration of flux.

\subsubsection{Membrane Activation Parameters}

The average flux rates of $\mathrm{KCl}_{a q}$ through identically prepared $\mathrm{PPy} / \mathrm{TOS}^{-}$membranes were compared for different cycling potential pulse widths, which are expected to affect the active transport efficiency of the membranes (Figure 4.13). An EIS probe was used to detect the concentration of $\mathrm{KCl}_{a q}$ in the receiving solution as a $\pm 1 \mathrm{~V}$ cycling potential was simultaneously applied at the membrane. Flux rates represent the amount of $\mathrm{KCl}_{a q}$ to have passed through the membrane in the 100 seconds following visible membrane "opening". 
Pulse widths of 10, 30, 50, 70 and 90 seconds were tested. A significant difference is seen between both 10 and 50 and 90 seconds and the 30 and 70 second cycling conditions $(\mathrm{p}<0.05)$. However, no difference exists between the 10, 50 and 90 second cycling conditions, or between 30 and 70 seconds $(\mathrm{p}>0.1)$. Clearly, these data are highly nonlinear as a function of cycling rate, with a possible resonance trend emerging from these data, where 10, 50 and 90 seconds seemingly show improved flux rates. However, more experiments are needed to determine if this trend is real and if there is an optimal peak resonance frequency for membrane activation. Regardless, these data hint at complex mechanisms for transport that should be investigated in future work. For the purpose of investigating flux rates though PPy membranes, and to remain comparable to literature $[78,146,154,160]$, the 50 second pulse width was selected for all subsequent flux experiments. 


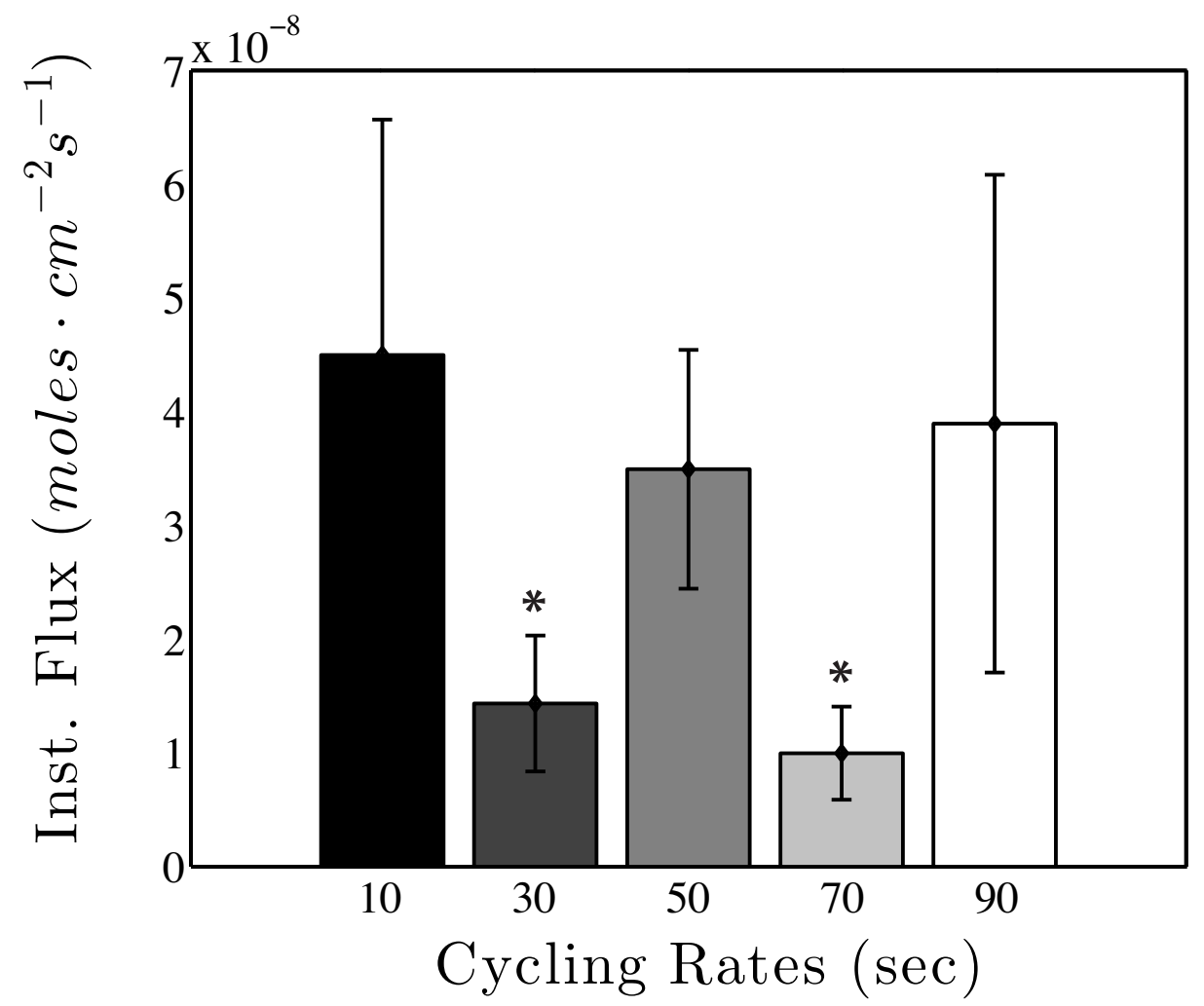

FIgURE 4.13: The instantaneous flux rates of $\mathrm{KCl}_{a q}$ through membranes produced under identical conditions $\left(2 \mathrm{~mA} / \mathrm{cm}^{2}\right.$ current density for 35 minutes) are shown at either 10, 30, 50, 70 or 90 second pulse widths during voltage cycling. An EIS probe was used to quantify $\mathrm{KCl}_{a q}$ concentration in the receiving solution simultaneous to voltage cycling at the membrane to allow ion passage. These data represent the ionic flux through $\mathrm{PPy}_{\mathrm{TOS}}{ }^{-}$during the 100 seconds following visible cycling initiation. The 10, 50 and 90 second pulse widths produces significantly better fluxes than the 30 and 70 seconds pulse widths $(\mathrm{p}<0.05)$. However, there is no significant difference between 10,50 and 90 second pulsing, or between 30 and 70 seconds $(\mathrm{p}>0.1)$.

\subsubsection{Membrane Performance Variability}

The flux performance of membranes produced in our lab within the same solution preparation, synthesis conditions, and within a few hours of one another was comparable (see Figure 4.14). In contrast, however, membrane groups produced on different days displayed wide variability (see Figure 4.14, comparing sets of three membranes). Although precautions were taken during synthesis to minimize variabilities in membrane morphologies, the exact cause for the observed fluctuations is unknown. It is hypothesized that pyrrole monomer age since distillation likely affects the membrane integrity significantly. As they age, pyrrole solutions self-oxidize in the presence of air 
and light, producing short polypyrrole strands in solution (turning the pyrrole solution dark as their concentration increases). The presence of these polymer strands in the preparation solution, it is hypothesized, could affect membrane integrity by the incorporation of these short polymer strands into the PPy, creating localized defects in the membrane. These defects would in turn affect electron transfer through the disrupted membrane and reduce the membrane's redox efficiency [69], making it very important to distill pyrrole prior to every experiment.

It is noteworthy that $\mathrm{PPy}$ membrane performance variability is a common criticism of these materials within the literature, and no universal synthesis conditions to prevent this occurence have yet been established [164-166]. Nonetheless, these results underscore the need for multiple experimental observations, from different days, to draw meaningful comparisons between data sets. As a result, the data analysis was always performed on membranes from different days to ensure validity in results. 


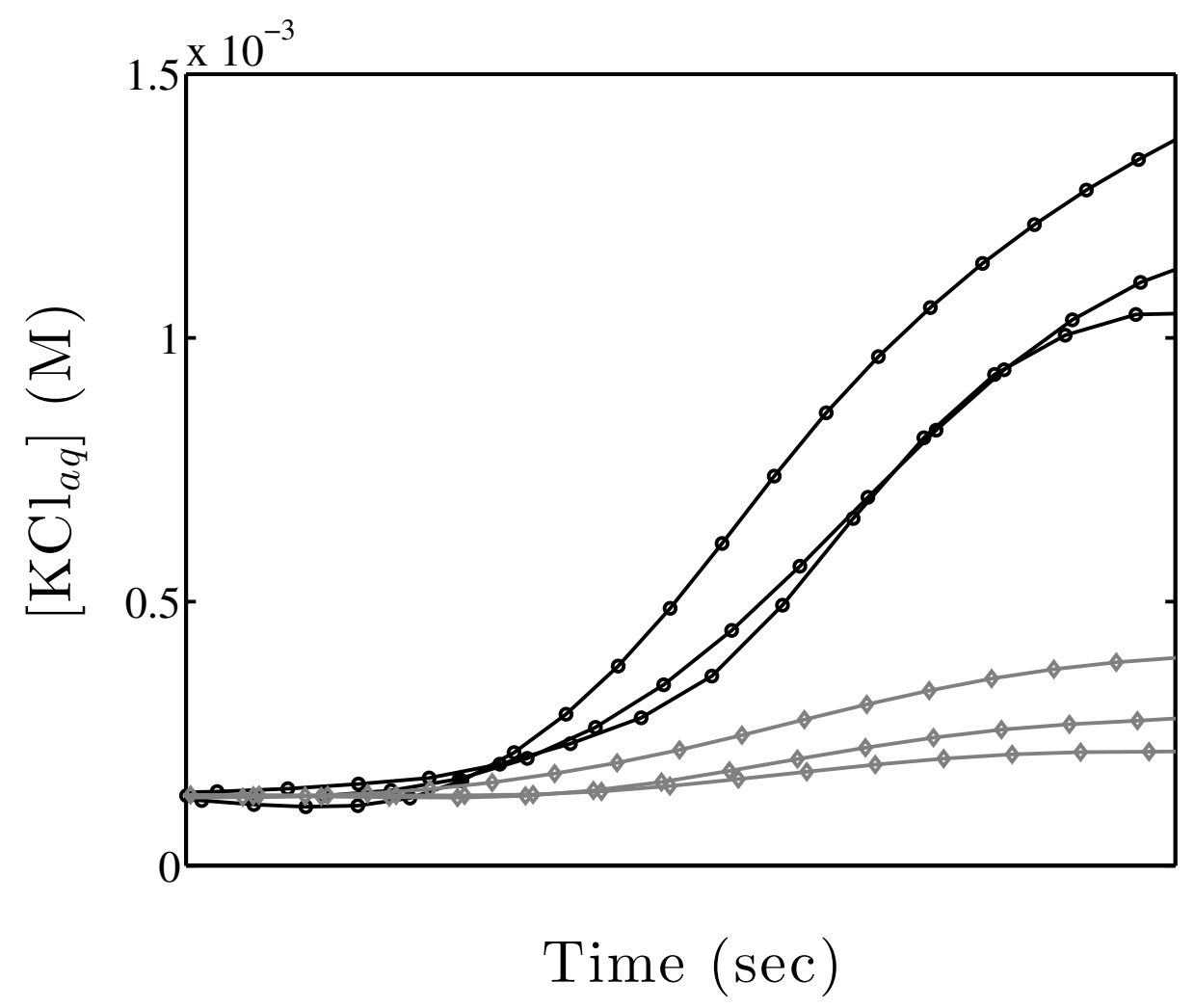

FIGURE 4.14: Concentration profiles of potassium chloride over time in the receiving solution for two groups of identical membranes produced on separate days. The first three $\mathrm{PPy} / \mathrm{TOS}^{-}$membranes (○) were produced in the same deaerated pyrrole solution, one after the other, and tested the same day. These membranes produce internally consistent flux rates. The next three membranes $(\diamond)$ were produced the next day, in a fresh solution from the same distilled pyrrole stock under the same conditions. Although these three curves are also internally consistent, they show reduced flux rates compared to the previous day. These data demonstrate the day to day performance variability which exists between these membranes.

Furthermore, a significant decrease in membrane performance was observed when the same membrane was used twice; once before and again after soaking in $\operatorname{diH}_{2} \mathrm{O}$ overnight (see Figure 4.15). On the second day, the membrane not only underperformed, but appeared to lose the ability to pass ions entirely shortly after the application of a cycling voltage. PPy membrane aging has also been described by other groups [167], with reports of membrane degradation over time being due to oxidative cycling [165]. In fact, Cheung et al. described how oxidation voltages in excess of 0.7 $\mathrm{V}$ vs. SCE on polypyrrole caused irreversible damage to the membrane, including loss of mass [168]. However, the CVs of the current membranes (Figure 4.1) do not show 
the severe hysteresis produced by electrochemical degradation, as shown in Cheung et al.'s PPy membranes after even a single cycle through overoxidative potentials [168]. The variation in their response may be a function of the materials used for their preparation, including a non-aqueous acetonitrile base and different counterion. Nonetheless, it remains possible that the membranes tested here may be experiencing some level of degradation over the cycling period, leaving them susceptible to further oxidative degradation overnight. In addition to oxidative cycling damage, another possible explanation for this behavior is ion retention in the membrane from the previous day's experiment, as suggested by Majumdar et al. [158]. These experiments shed light on the limited lifespan of PPy membranes and that is a characteristic that should be taken into consideration for the long-term viability of $\mathrm{PPy}$ as an artificial cell membrane. 


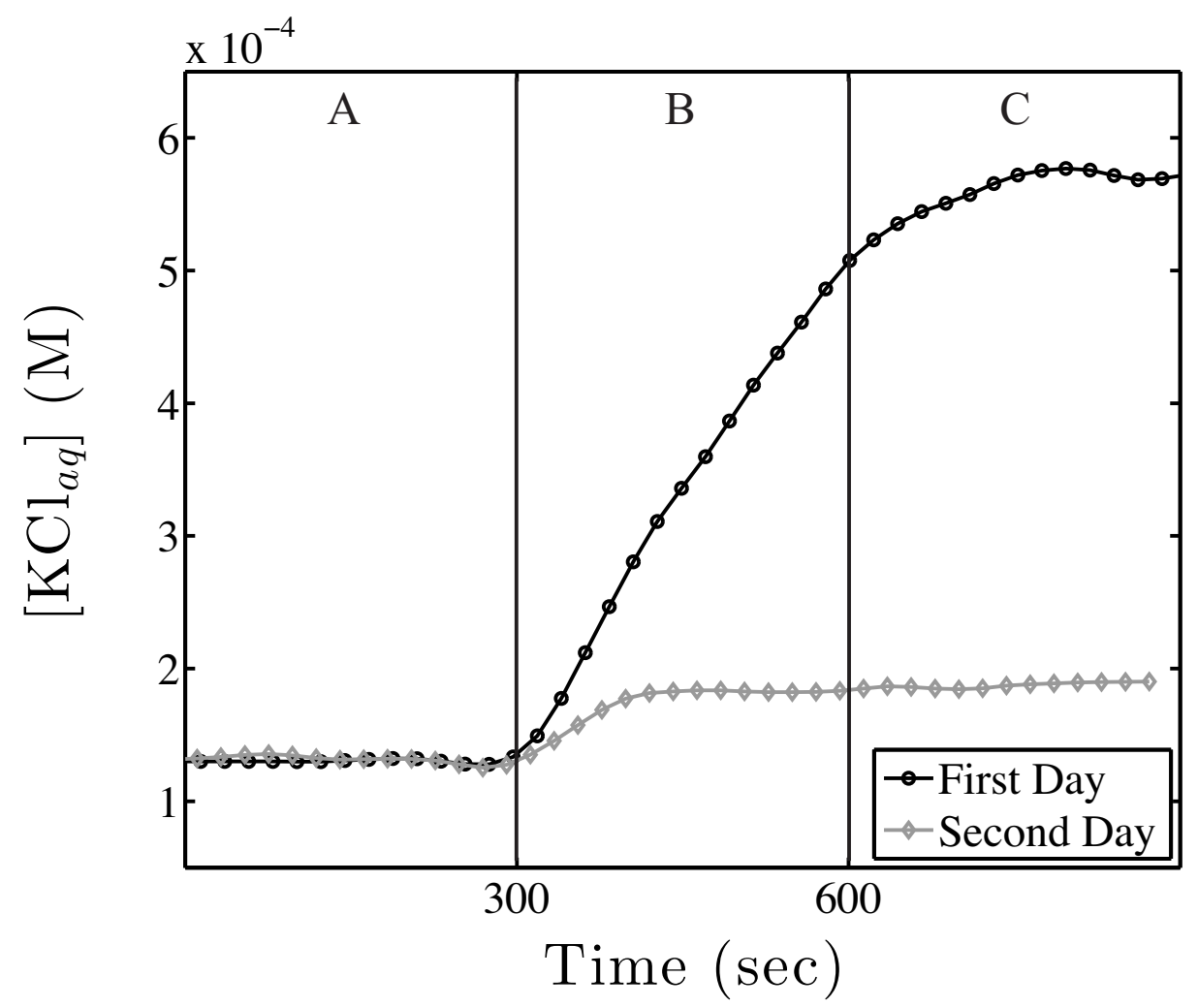

Figure 4.15: An example flux comparison for the same membrane tested on two consecutive days is shown. A PPy/TOS ${ }^{-}$membrane produced at $2 \mathrm{~mA} / \mathrm{cm}^{2}$ for 35 minutes, was activated with \pm 1 $\mathrm{V}, 50$ second pulse width cycling on the first day $(\bigcirc)$. The membrane was stored in $\mathrm{diH}_{2} \mathrm{O}$ overnight, and the exact same conditions were applied the next day $(\diamond)$. A visible and significant change in performance is observed. A) Membrane is at rest. B) A cycling potential applied at membrane and C) at rest again.

In summary, the time-resolved concentration readings of $\mathrm{KCl}_{a q}$ demonstrate some important information regarding the PPy membrane flux rates, including performance variability from day to day, and within the same membrane after multiple uses. It was also determined that ion movement across the membrane in solution is delayed by the time needed to complete a full oxidation / reduction cycle on the PPy, which should be considered in the calculation of flux rates. Membranes were also found to have nearly linear flux rates after initial opening, which justifies the use of time-lapse measurements of the ion concentration in solution before and after the application of a cycling potential, so long as the delayed onset flux is taken into consideration. 


\subsubsection{Time-Lapse Concentration Readings in Solution}

Unfortunately, real-time measurement of $\mathrm{KCl}_{a q}$ as it moves through hydrogel encapsulated PPy membranes is not feasible. The presence of hydrogel created a feedback mechanism between the two simultaneous potentiostats and introduced significant noise into the EIS readings. Instead, the flux of ions in this scenario was measured via EIS time-lapse, similar to the discretized measurement methods used by Akieh et al. $[154,160]$ and many others. EIS time-lapse readings performed in this manner related $\mathrm{KCl}_{a q}$ concentration readings without the need to remove aliquots from the receiving solution.

EIS time-lapse of $\mathrm{KCl}_{a q}$ concentrations were obtained using a single potentiostat to control both the membrane oxidation state of PPy, and the EIS probe sequentially. This method of switching back and forth between measuring the concentration of $\mathrm{KCl}_{a q}$ and controlling voltage application at the membrane produced the concentrations profiles of $\mathrm{KCl}_{a q}$ seen in Figure 4.16. From the time-resolved flux curves obtained in the previous section, we know the slope of ion transport produced by time-lapse measurement is oversimplified. However, flux rates calculated on these slopes are easily corrected to reflect this discrepancy, by using $\Delta \mathrm{t}=500$ instead of 600 seconds in Equation 4.4. Flux rates obtained using this method were then used to compare membranes produced at different current densities, as will be discussed next. 


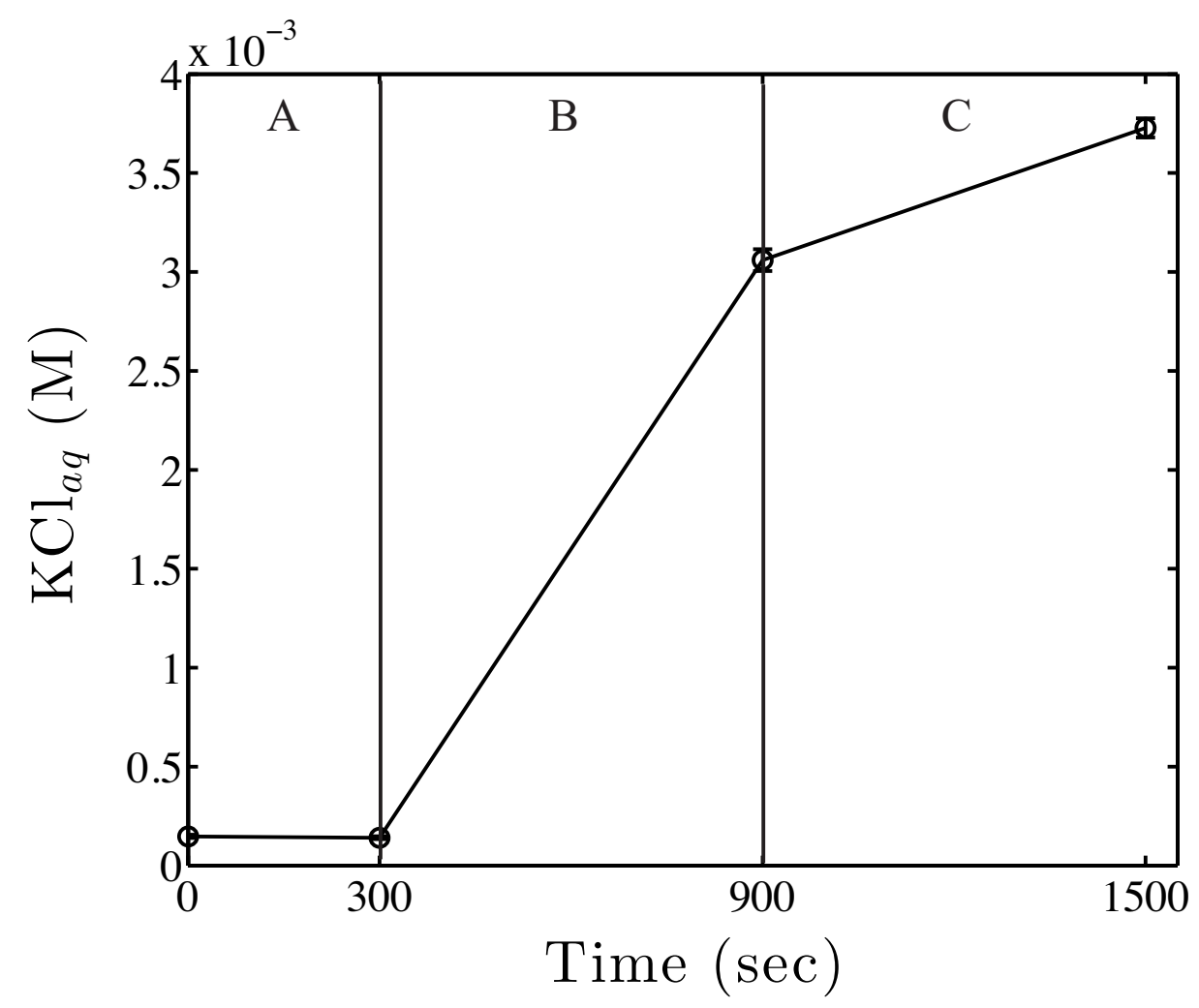

Figure 4.16: An example time-lapse concentration profile for $\mathrm{KCl}_{a q}$ in receiving solution is shown versus time, as it travels through a PPy/TOS ${ }^{-}$membrane $\left(5 \mathrm{~mA} / \mathrm{cm}^{2}\right.$ for 15 minutes $)$. By using a single potentiostat to alternatively measure solution conductivity using the EIS probe, and apply a voltage at the membrane, discretized concentration results were obtained. A: The membrane is at rest and no ionic leakage is observed after the addition of $1 \mathrm{M} \mathrm{KCl}_{a q}$ to the feed chamber. B: The membrane is cycled at $\pm 1 \mathrm{~V}$ with 50 second pulse widths for 10 minutes, at the end of which an EIS recording measures the potassium chloride concentration in solution. C: The membrane receives a $-1 \mathrm{~V}$ potential to 'close'.

\subsubsection{Comparing Membrane Deposition Schemes}

The effect of membrane deposition conditions on the flux of $\mathrm{KCl}_{a q}$ through PPy/TOS membranes was studied by use of two deposition schemes, $\mathrm{PPy}_{a}$ and $\mathrm{PPy}_{b}$, which were previously described and summarized below in Table 4.1. $\mathrm{PPy}_{a}$, which was deposited at $5 \mathrm{~mA} / \mathrm{cm}^{2}$ for 15 minutes, resulted in a $9.3 \mu \mathrm{m}$ thick membrane, while $\mathrm{PPy}_{b}$ was deposited at $2 \mathrm{~mA} / \mathrm{cm}^{2}$ for 25 minutes, and resulted in a slightly more dense and thicker membrane (see Table 4.1).

Flux rates obtained across these two different membranes under identical concentration gradients and activation conditions are shown in Figure 4.17 below. As seen, 
TABle 4.1: $\mathrm{PPy}_{a}$ and $\mathrm{PPy}_{b}$ : Membrane Summaries

\begin{tabular}{|c|c|c|}
\hline & $\mathrm{PPy}_{a}$ & $\mathrm{PPy}_{b}$ \\
\hline Current Density & $5 \mathrm{~mA} / \mathrm{cm}^{2}$ & $2 \mathrm{~mA} / \mathrm{cm}^{2}$ \\
\hline Duration & 15 mins & 25 mins \\
\hline Thickness, $t$ & $9.3 \mu \mathrm{m}$ & $9.6 \mu \mathrm{m}$ \\
\hline Density, $\rho$ & $1.456 \mathrm{~g} / \mathrm{cm}^{3}$ & $1.461 \mathrm{~g} / \mathrm{cm}^{3}$ \\
\hline Flux, $\phi$ & $0.26 \times 10^{-7}$ moles $/ \mathrm{cm}^{2} \cdot s$ & $1.34 \times 10^{-7}$ moles $/ \mathrm{cm}^{2}$ \\
\hline
\end{tabular}

there is a significant difference between the flux rates obtained through these two membranes. Since the difference between $\mathrm{PPy}_{a}$ and $\mathrm{PPy}_{b}$ is arguably negligible in terms of their membrane thickness and density, these data point to underlying structural polymer differences in the membranes affecting flux rates, which are a result of deposition conditions. These data and their significance will be discussed in more detail in the next chapter. 


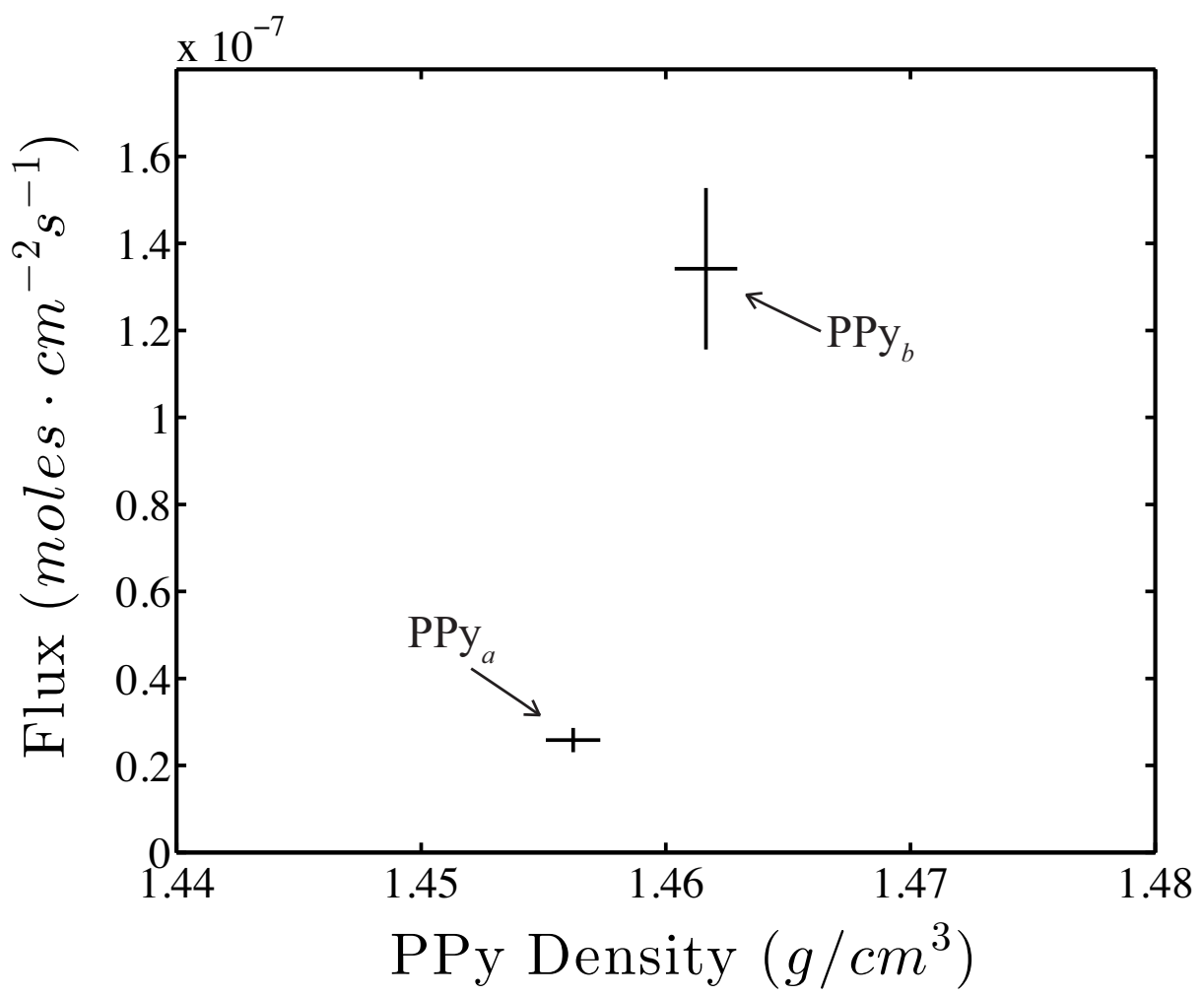

FiguRE 4.17: Flux rates of $\mathrm{KCl}_{a q}$ through $\mathrm{PPy} / \mathrm{TOS}^{-}$membranes prepared in two separate ways are compared. PPy $: 5 \mathrm{~mA} / \mathrm{cm}^{2}$ deposition for 15 minutes $\left(9.3 \mu \mathrm{m}\right.$ thick). $\mathrm{PPy}_{b}: 2 \mathrm{~mA} / \mathrm{cm}^{2}$ deposition for 25 minutes (9.6 $\mu \mathrm{m}$ thick). Membrane densities measured by Micromeritics Analytical Services $(\mathrm{n}=10)$, with $\mathrm{x}$-direction standard error shown. A 50 second pulse width at $\pm 1 \mathrm{~V}$ vs. SCE were applied for 10 minutes on each membrane, and flux rates across the membrane are shown with respective $\mathrm{y}$-direction standard errors $(\mathrm{n}=6)$. It is interesting to note that despite having similar density and thickness, $\mathrm{PPy}_{b}$ demonstrates a significantly higher flux for $\mathrm{KCl}_{a q}$ than $\mathrm{PPy}_{a}$.

\subsubsection{Comparing Concentration Gradients}

The effects of increased concentrations of $\mathrm{KCl}_{a q}$ in the feed solution were tested to observe the possible effects of salt retention between cycling events in the membrane. Flux rates were compared through $\mathrm{PPy}_{b}$ membranes cycled under identical conditions $( \pm 1 \mathrm{~V}$ and 50 second pulse widths, $\mathrm{n}=3$ ), and their results reported in Table 4.2 below.

As evident from these data, the conditions tested offer no significant difference in flux rates as a result of feed solution $\mathrm{KCl}_{a q}$ concentrations, in the ranges of $1 \mathrm{M}$ to 4 $\mathrm{M} \mathrm{KCl}_{a q}$ tested. This indicates that the effects of residual salts, in the range tested, 
TABle 4.2: Flux Rate Comparison: $\mathrm{PPy}_{b}$ in Varying Feed Solutions

\begin{tabular}{|c|c|c|c|}
\cline { 2 - 4 } Flux & \multicolumn{3}{|c|}{ Feed Concentration $\left(\mathrm{KCl}_{a q}\right)$} \\
\cline { 2 - 4 } & $1 \mathrm{M}$ & $2 \mathrm{M}$ & $4 \mathrm{M}$ \\
$\left.\begin{array}{c}\text { Standard Error } \\
\text { Stanoles }\end{array} \mathrm{cm}^{2} \cdot s\right)$ & $1.34 \times 10^{-7}$ & $1.73 \times 10^{-7}$ & $1.55 \times 10^{-7}$ \\
\cline { 2 - 4 } & $3.68 \times 10^{-8}$ & $2.39 \times 10^{-8}$ & $7.75 \times 10^{-8}$ \\
\cline { 2 - 4 }
\end{tabular}

do not interfere with the gating abilities of $\mathrm{PPy} / \mathrm{TOS}^{-}$. It is also interesting to note that there appears to be a saturation limit for flux of ions through $\mathrm{PPy}_{b}$, which was reached already at $1 \mathrm{M} \mathrm{KCl}_{a q}$. A purely diffusive membrane would offer increased flux rates in the presence of increased concentration gradients. Since it is not the observed case here, it lends further confirmation of the importance of the active binding and release mechanism for ion transport through these membranes.

\subsubsection{Time-Lapse Concentration Readings in PPy / Hydro- gel}

The next step towards preparing an artificial excitable cell membrane is to demonstrate the functionality of PPy membranes in the presence of a hydrogel environment. From the results obtained on $\mathrm{PPy}$ membranes in solution, the $\mathrm{PPy}_{b}$ membrane deposition scheme was chosen for testing in the hydrogel environment since it produced the fastest flux rates. Flux experiments were therefore performed on a $\mathrm{PPy}_{b} /$ hydrogel sandwich structure as shown in Figure 4.18, with results reported below. 


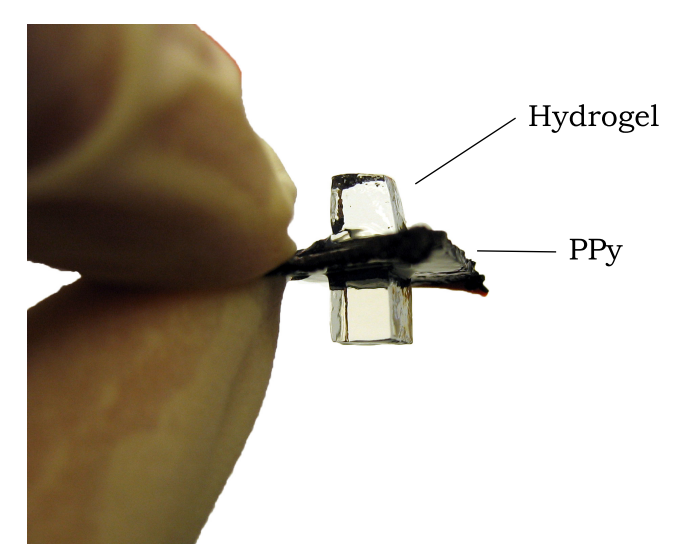

Figure 4.18: A PPy/TOS ${ }^{-}$membrane is shown encapsulated by two hydrogels. These structures were used in the experimental setup to measure the flux rate of $\mathrm{KCl}_{a q}$ through the encapsulated membrane.

A time-lapse concentration profile of $\mathrm{KCl}_{a q}$ as it diffuses into the receiving gel of a $\mathrm{PPy}_{b} /$ hydrogel structure with $2 \mathrm{~nm}$ pore size $\left(\mathrm{PPy}_{b} /\right.$ hydrogel $\left._{2 n m}\right)$ is shown, before and after cycling (Figure 4.19). The continued flux of ions observed after membrane closing (Region $\mathrm{C}$ ), which differs from the immediate flux dampening observed after membrane closing in solution, is hypothesized to be due to a diffusion lag induced by the presence of the hydrogel. Indeed, it is known that the diffusivity constant for $\mathrm{KCl}_{a q}$ is lower in hydrogel than in solution [98], which would account for the ion pair taking longer to reach equilibrium in the hydrogel after crossing the PPy membrane. Flux rates for Regions B and C in Figure 4.19 were calculated to be $7.96 \times 10^{-10}$ and $10.84 \times 10^{-10}$ moles $/ \mathrm{cm}^{2} \cdot s$, respectively, and their difference was not found to be statistically significant $(\mathrm{p}>0.1)$. These data demonstrate that membrane closing in hydrogel, therefore, does not immediately stop ion migration through the hydrogel, and ions are still reaching equilibrium throughout the gel up to 10 minutes after membrane closing. 


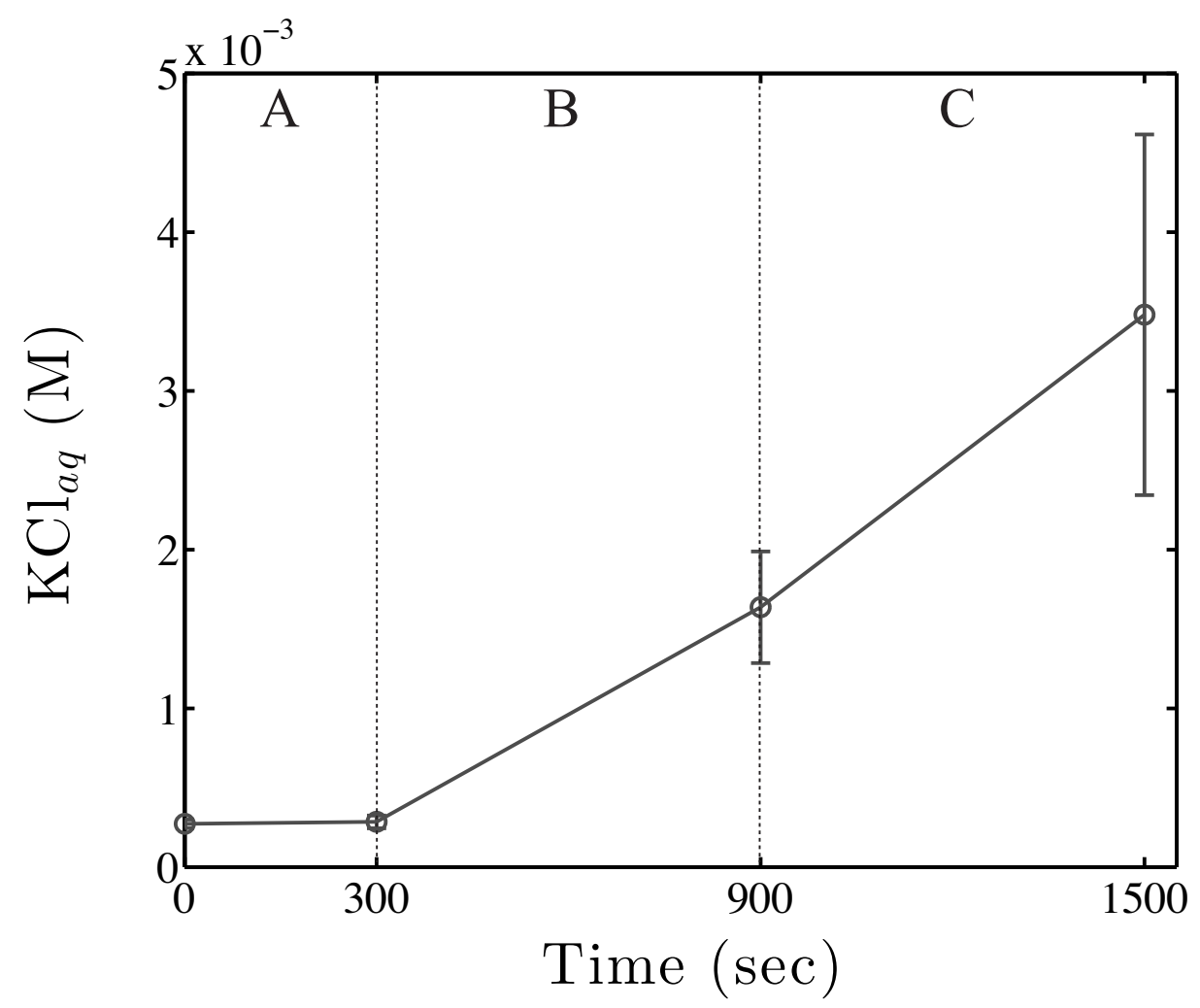

FiguRE 4.19: Time-lapse concentration profile for $\mathrm{KCl}_{a q}$ in receiving hydrogel is shown versus time, as it travels through a $\mathrm{PPy}_{b} /$ hydrogel $_{2 n m}$ structure $(\mathrm{n}=3)$. A: The membrane is at rest and no ionic leakage is observed after the addition of $1 \mathrm{M} \mathrm{KCl}_{a q}$ to the feed chamber. B: The membrane is cycled at $\pm 1 \mathrm{~V}$ with 50 second pulse widths for 10 minutes. C: The membrane receives a $-1 \mathrm{~V}$ potential to 'close'. Flux rates in Regions B and C were calculated to be $7.96 \times 10^{-10}$ and $10.84 \mathrm{x}$ $10^{-10} \mathrm{moles} / \mathrm{cm}^{2} \cdot s$, respectively.

The flux rates of $\mathrm{KCl}_{a q}$ as a function of varying hydrogel pore sizes $(0.1 \mathrm{~nm}, 0.5$ $\mathrm{nm}$ and $2 \mathrm{~nm}$ ) are shown in Figure 4.20 below for hydrogel covered $\mathrm{PPy}_{b} / \mathrm{TOS}^{-}$ membranes. A linear trend between flux rate and hydrogel pore size is observed, with flux rates increasing with pore size. The transport rates of potassium chloride through 0.5 and $2 \mathrm{~nm}$ hydrogel covered membranes were compared using a KruskalWallis ANOVA, and determined to be significantly different $(\mathrm{p}<0.05)$. However, there is no significant difference between the 0.1 and $0.5 \mathrm{~nm}$ pore size flux rates. 


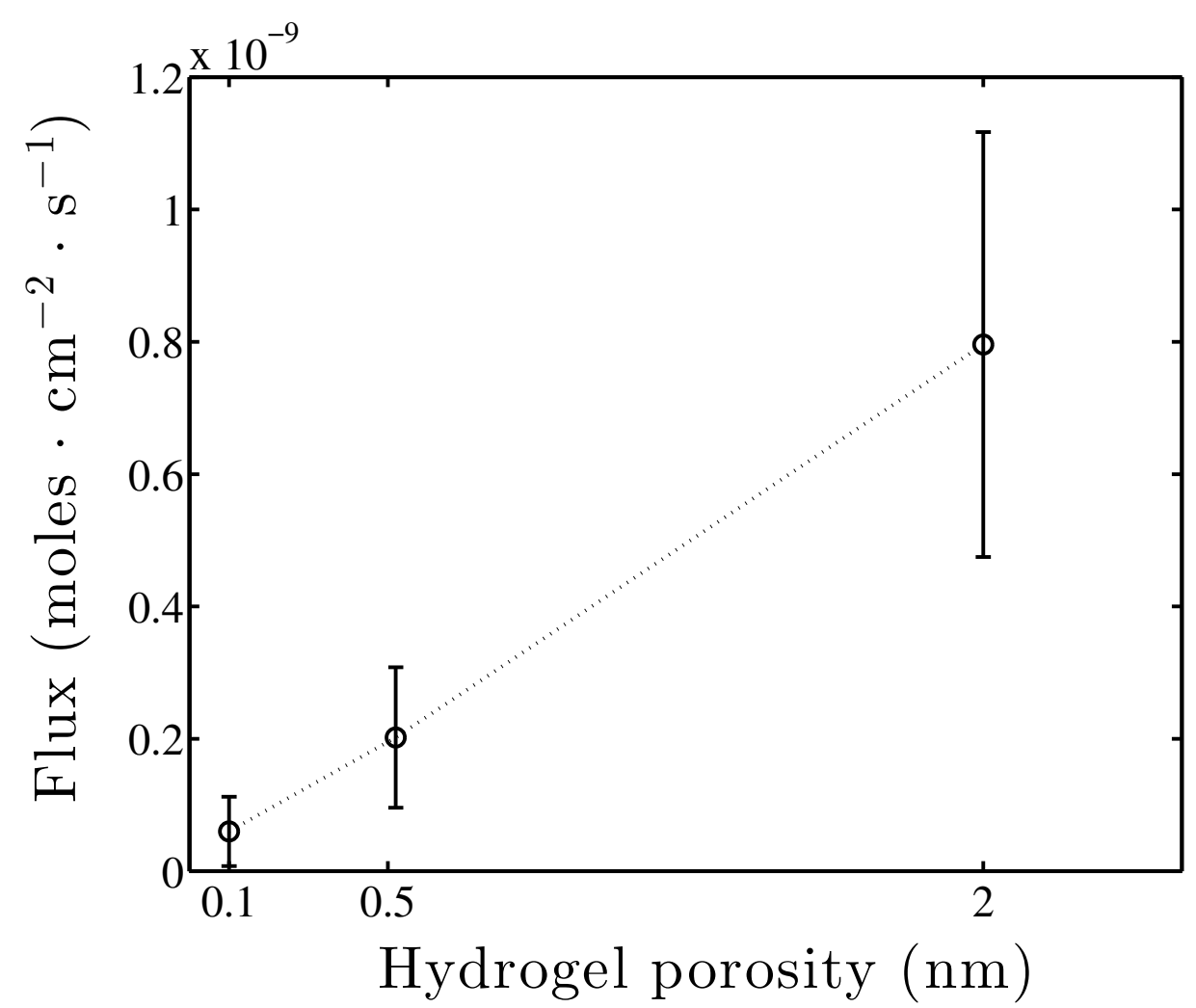

Figure 4.20: Flux rates of $\mathrm{KCl}_{a q}$ through hydrogel encapsulated $\mathrm{PPy} / \mathrm{TOS}^{-}$membrane stacks are shown as a function of pore size. Clearly, flux rates are highly dependent on hydrogel pore size. However, even at $2 \mathrm{~nm}$ pore size, the flux rates through $\mathrm{PPy}_{b} / \mathrm{TOS}^{-}$are still two orders of magnitude lower than for identical conditions applied in solution. Standard error bars shown, $\mathrm{n}=$ 3 for each pore size.

The purpose for using hydrogel to encase the ion-gating PPy membrane was to produce a semi-solid containment region in which ions are free to move, mimicking biological intra- and extracellular spaces. However, the average flux rate through the $\mathrm{PPy}_{b} /$ hydrogel ${ }_{2 n m}$ structure was reduced by over two orders of magnitude compared to flux rates through the same membrane in solution alone (see Table 4.3).

TABLE 4.3: Flux Rate Comparison: $\mathrm{PPy}_{b}$ in Solution and Hydrogel

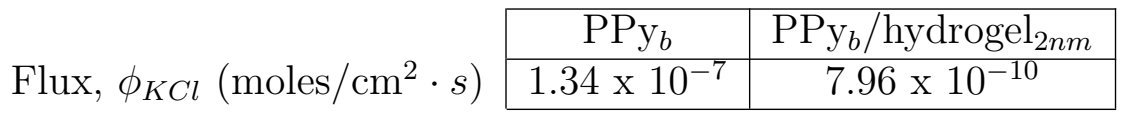

These data confirm that polypyrrole membranes retain their ion gating ability in the new hydrogel environment. The presence of the hydrogel, however, slows ionic 
flux through the membrane by two orders of magnitude, due to the lower diffusivity of $\mathrm{KCl}_{a q}$ in the porous network. This also causes the $\mathrm{PPy}_{b} /$ hydrogel system to appear insensitive to membrane closing, showing significantly delayed response as compared to solution. The variable hydrogel porosity, nonetheless, offers an additional degree of control over flux rates through the polymer network, which can be used as slow-return valves as described in the conceptual gel-cell design. These results demonstrate the feasibility of using this system as an artificial cell membrane.

\subsection{Potassium Ion Selective Electrode}

Having established the feasibility of the ionic gate as a basic artificial cell membrane, the focus turns to the ability to make this membrane excitable. A potassium sensor (ISE, see Figure 3.9), as previously discussed, is critical for accomplishing this in two areas of the artificial cell. It will be needed within the ion switch to trigger membrane opening, and again to detect potassium ions once they pass through the membrane as a marker for cell activation. Since the ISE typically produces a voltage drop with respect to a reference electrode, it was hypothesized that a voltage drop could be produced between the two ISEs within the artificial cell instead, to simplify the artificial cell membrane design (See Figure 4.21). Experiments demonstrating feasibility are shown below. 


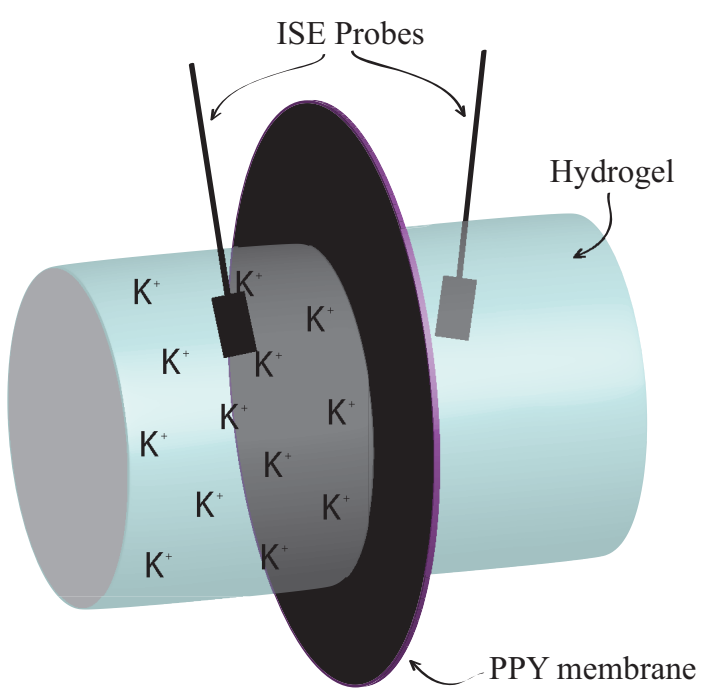

FiguRE 4.21: Schematic representation of the ISE probes placed on either side of a PPy membrane. This configuration is hypothesized to produce voltage drops across the membrane in response to $\mathrm{KCl}_{a q}$ concentrations.

ISE sensors were tested opposite one another in a variety of combinations, as listed in Table 4.4. First, a voltage drop was read between an ISE electrode placed in $1 \mathrm{M} \mathrm{KCl}$ and reference electrode in buffer, as a positive control (Table 4.4, a). Next, two ISE electrodes were used instead within the same configuration as before. Surprisingly, the voltage drop produced between the two ISE electrodes was larger than between ISE and reference electrode (Table 4.4, b). To further investigate the feasibility of this setup in the context of the gel-cell, where there would be no buffer solution, in c) an ISE sensor was placed in $1 \mathrm{M} \mathrm{KCl}_{a q}$ and read relative to a low concentration $\mathrm{KCl}$ solution instead (i.e. $10^{-4} \mathrm{M} \mathrm{KCl}_{a q}$ ). As expected, the voltage drop response in this scenario decreased significantly, by an order of magnitude (Table $4.4, \mathrm{c})$. 
TABLE 4.4: ISE Recordings

\begin{tabular}{l|c|c|c|}
\hline \multicolumn{1}{c|}{ Electrodes } & Setup & Voltage Drop (V) \\
\cline { 2 - 4 } a) & ISE vs. RE & $1 \mathrm{M} \mathrm{KCl}_{a q}$ vs. Buffer & 1.1783 \\
\cline { 2 - 4 } b) & ISE vs. ISE & $1 \mathrm{M} \mathrm{KCl}_{a q}$ vs. Buffer & 1.5412 \\
\cline { 2 - 4 } c) & $\mathrm{ISE}$ vs. ISE & $1 \mathrm{M}$ vs. $10^{-4} \mathrm{M} \mathrm{KCl}_{a q}$ & 0.1866 \\
\cline { 2 - 4 } d) & $\mathrm{ISE} / \mathrm{Hyd}_{2 n m}$ vs. ISE $/ \mathrm{Hyd}_{2 n m}$ & $1 \mathrm{M}$ vs. $10^{-5} \mathrm{M} \mathrm{KCl}_{a q}$ & 0.6672 \\
\cline { 2 - 4 } & \multicolumn{2}{|l}{} \\
\multicolumn{2}{|l}{}
\end{tabular}

Finally, PPy sensors were molded into $2 \mathrm{~nm}$ pore size hydrogels and investigated using a new test setup, depicted in Figure 4.22. For this setup, two hydrogel covered PPy sensors were pre-soaked in $1 \mathrm{M} \mathrm{KCl}$ and $10^{-5} \mathrm{M} \mathrm{KCl}$, respectively, and placed into contact with one another. The voltage measured over time across these PPy sensors is shown in Figure 4.23, and the calculated voltage drop is reported in Table 4.4 , d.
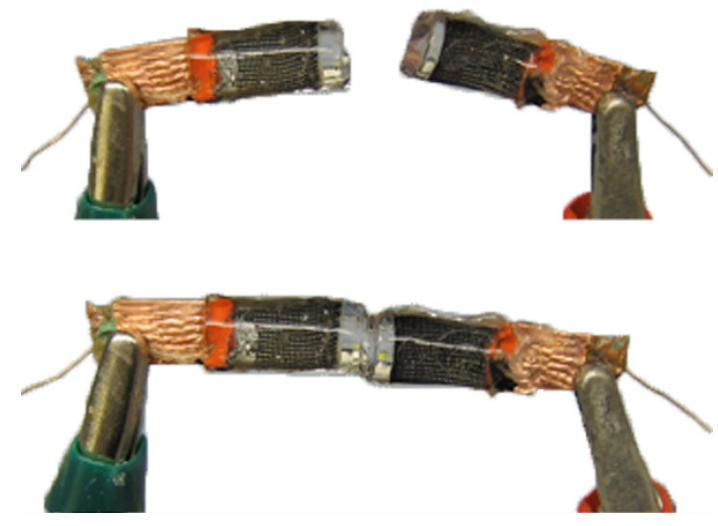

FIGURE 4.22: Hydrogel covered ISE sensors are shown, before and after being placed in contact. A voltage drop was recorded between the two sensors, as one was pre-soaked in $1 \mathrm{M}$ and the other in $10^{-5} \mathrm{M} \mathrm{KCl}_{a q}$ for 24 hours prior to testing. 


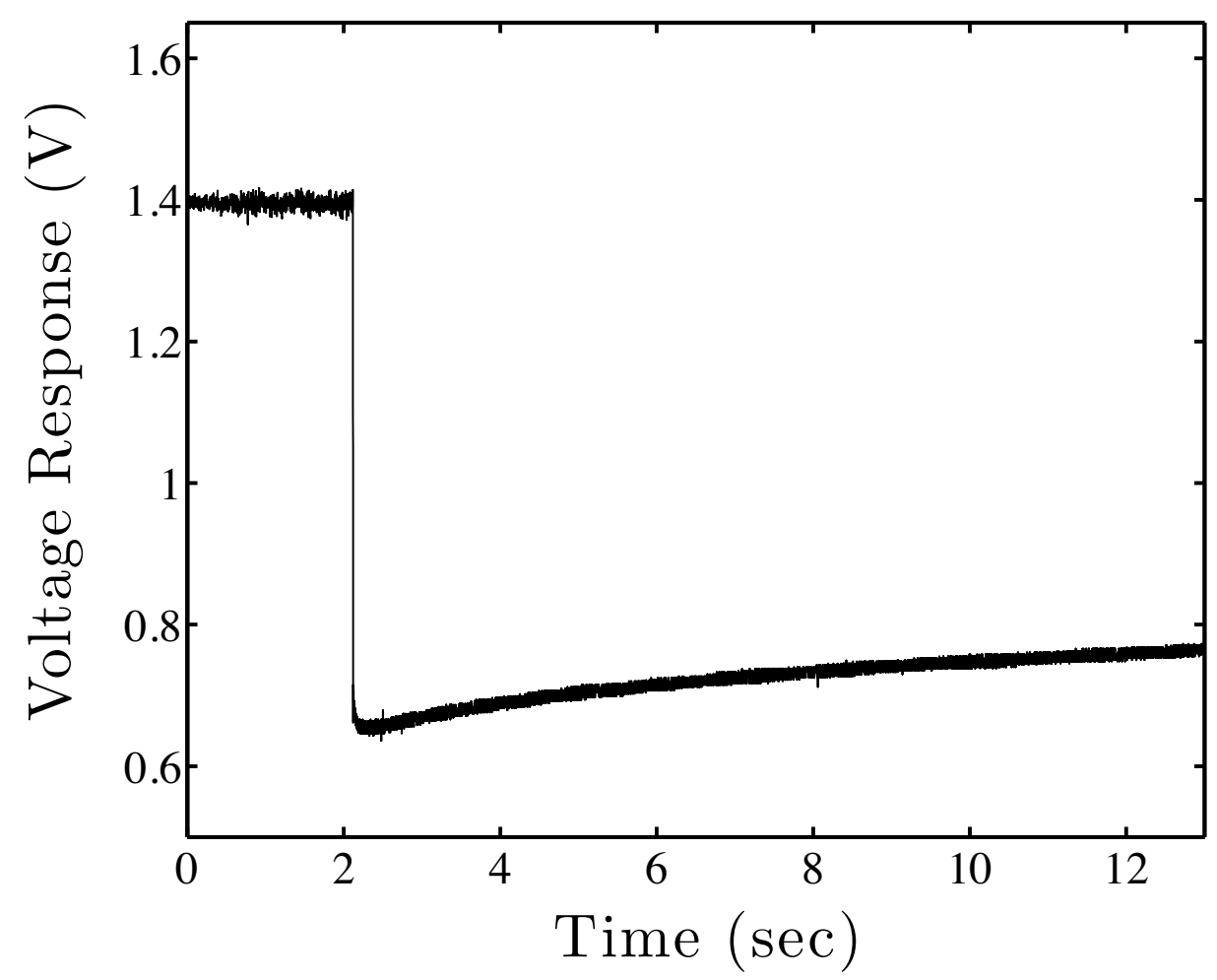

Figure 4.23: Example voltage drop readings over time for hydrogel covered ISE probes coming into contact with one another around the 2 second mark. One ISE sensor was pre-soaked in $1 \mathrm{M}$ and the other in $10^{-5} \mathrm{M} \mathrm{KCl}_{a q}$ for 24 hours before testing. The gradual upward sloping of the voltage response indicates the flux of ions redistributing to equilibrium between the two hydrogels after contact. The default output from the voltage reader was filtered using median and Loess filters to reveal a baseline reading of $1.4 \mathrm{~V}$ in the absence of an external voltage.

The slow increase in voltage seen in Figure 4.23 is a result of the migration of potassium ions across the hydrogels as they tend towards an equilibrium between the high and low concentration hydrogels.

These results establish that ISE sensors can be used in the artificial cell assembly to detect the presence of potassium ions in a simplified configuration, and in fact, appear to exhibit improved performance in the hydrogel environment. A spectrum calibration of the relative voltages should be performed, and is recommended for future work. 


\section{Chapter 5}

\section{Discussion}

This chapter examines the suitability of both polypyrrole and hydrogel materials as artificial cell components. In the first section, polypyrrole's potential as a biomimetic artificial cell membrane is considered, with particular attention to the maximum flux rates obtained, and their analogy to voltage-gated ion channels. In the following section, hydrogel is examined as a potential artificial intra- and extra-cellular matrix analog, with emphasis on the flux results obtained through the PPy/Hydrogel stacks, and how these compare to biology.

\subsection{Polypyrrole as an Artificial Cell Membrane}

Polypyrrole is an electroactive polymer material capable of reversibly allowing the passage of ions in response to voltage stimulus, analogous to the voltage-gated ion channels found in biological cell membranes. To the best of the knowledge of the author, no prior research exists aimed at using PPy as an artificial excitable cell membrane, despite their excellent gating properties and high flux rates.

Several groups have, however, modeled voltage-gated ion channels using pores in a variety of different polymers (none of which are PPy) [169-172]. For example, one group created $\mathrm{K}^{+}$-gated titania nanotubes capable of blocking the flow of large anions 
through the membrane in the presence of potassium ions [173]. Yet another produced synthetic voltage-gated ion channels from conical pores in polymer [174]. Many examples exist of artificial VGICs where ionic flux rates through these structures were not provided, offering proof-of-concepts without being directly comparable to biological transfer rates.

A few groups did provide flux rate data for solute passage. For example, a VGIC analogous membrane produced from carbon nanotubes was able to transfer large molecules at up to $6 \times 10^{-13}$ moles $/ \mathrm{cm}^{2} \cdot s$ [175]. Although the disparity in size between $\mathrm{KCl}$ and the molecules used in their study makes it impossible to compare flux rates directly, their flux rates were several orders of magnitude lower than those achieved through polypyrrole in this lab, as seen in Table 4.3. The flux rates of the PPy membranes in this work outperform any comparable membrane gating technologies currently available, making them ideal for use as an artificial ion gating membrane in the proposed gel-cell. The main results obtained on these membranes are discussed in more detail below.

\subsubsection{Ion Gating}

As stated by Majumdar et al., the optimal design of ion gating membranes involves maximizing their delivered flux rates and minimizing their leakage and retention rates [158]. Their minimum performance criteria, stated in 2011, called for leakage flux rates lower than $10^{-22}$ mole $/ \mathrm{cm}^{2} \cdot s$, and maximum delivered flux rates greater than $10^{-18}$ mole $/ \mathrm{cm}^{2} \cdot s$. By iterative optimization of a computational set of parameters involved in redox activation, Majumdar's group determined the maximum flux rate to be $1.74 \times 10^{-15}$ mole $/ \mathrm{cm}^{2} \cdot s$ through the membrane. Just a few months later in the same year, however, Akieh et al. [154] demonstrated flux rates for potassium $\left(\mathrm{K}^{+}\right)$through $\mathrm{PPy} / \mathrm{TOS}^{-}$membranes up to $3.6 \times 10^{-10} \mathrm{~mole} / \mathrm{cm}^{2} \cdot \mathrm{s}$, which are several orders of magnitude higher than predicted. Even better still, work shown here demonstrates molar flux rates through PPy $/ \mathrm{TOS}^{-}$, reaching $1.6 \times 10^{-7}$ mole $/ \mathrm{cm}^{2} \cdot \mathrm{s}$. 
These higher flux rates, however, are in part due to stronger driving concentration gradients across the membrane, which are expected to improve ionic conductivity across the membrane [176]. A source solution of $1 \mathrm{M} \mathrm{KCl}_{a q}$ was used in our experiments, whereas Akieh et al. used $0.1 \mathrm{M} \mathrm{KCl}_{a q}$ [154]. Inspecting Fick's First Law of diffusion, it is noted that diffusion flux is proportional to concentration gradient in Equation 5.1;

$$
J=-D \frac{\partial \phi}{\partial x}
$$

where $J$ is the flux, $D$ is the diffusivity, $\phi$ is the concentration and $x$ the position. From this equation we know that the concentration gradient increase in our experiments should produce no more than one order of magnitude increase in the resulting fluxes. However, the current results offer three orders of magnitude improvement over Akieh et al.'s flux rates, which is a significant improvement [154]. These excellent flux rates provided us the opportunity to investigate the effects of density and structure on the ionic conductivity of the membrane.

\subsubsection{Active Ion Pumping through Cycling}

The method by which polypyrrole thin membranes actively pump ions through is known as charge compensation "capture and release". Davey et al. [62] showed that p-toluenesulfonate $\left(\mathrm{TOS}^{-}\right)$, the counterion used for the preparation of PPy in these studies, is a large polyelectrolyte that lodges itself semi-permanently into the polymeric chain (see Figure 5.1). Upon electrochemical reduction (-1 V vs. SCE), the membrane initiates the incorporation of small positive ions $\left(\mathrm{K}^{+}\right.$ions in our case) to compensate for the negative charge accumulation within PPy (charge compensation capture). When the potential at the membrane is returned to positive through oxidation ( $+1 \mathrm{~V}$ vs. $\mathrm{SCE})$, the cations are then expelled from the membrane and move preferentially down their concentration gradient (charge compensation release). 
To maintain electroneutrality, negatively charged chloride ions are simultaneously entrained through the membrane with potassium ions to balance the flux of positive charges across the membrane. As a result of this capture and release mechanism, the largest flux of potassium chloride is seen during oxidation and reduction cycling as compared to a static voltage application at the membrane.

A

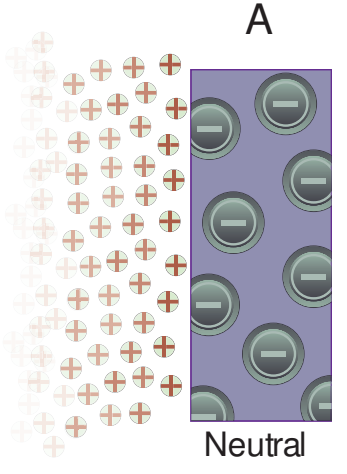

B

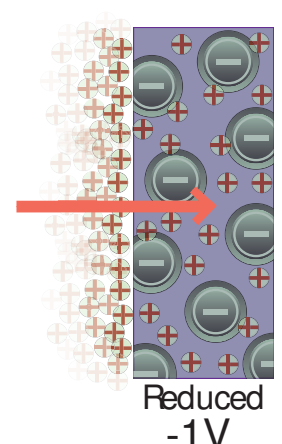

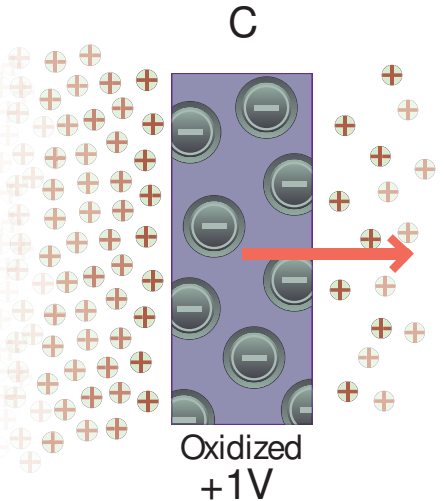

Figure 5.1: Graphical representation of ion pumping through a PPy membrane by potential cycling between oxidation and reduction. A: The membrane at rest is in the neutral state and a double layer forms at the surface in contact with the electrolyte solution. B: With the application of $-1 \mathrm{~V}$ vs. $\mathrm{SCE}$, the membrane is reduced. Once the energy barrier is overcome for the ions to break through the double layer, they incorporate into the membrane to charge neutralize the $\pi$-bonded sites. $\mathrm{C}$ : Upon the application of $+1 \mathrm{~V}$ vs. SCE the membrane is oxidized and ions are expelled down their concentration gradient.

In addition, the polarity of the first voltage applied at the membrane during cycling controls the delay observed before concentrations of $\mathrm{KCl}_{a q}$ become detectable, as seen in Figure 4.12. In this case, a positive voltage was initially applied at the membrane, followed by a negative voltage. The entire first $\pm 1 \mathrm{~V}$ cycle represents membrane loading with available ions, after which, the application of a positive voltage results in the first ions to be released into the receiving solution, which accounts for the observed response delays. This is easily accounted for in flux rate calculations, as described previously, by reducing the time span used to calculate flux rates in Equation 4.4 by the "membrane loading" time, or 100 seconds.

Flux rates of $\mathrm{KCl}_{a q}$ through the membrane are also highly dependent on membrane structure and properties. Figure 5.2 below depicts the primary, secondary and 
hypothesized tertiary structures of the compounds involved in the formation of PPy chains and membrane pores. As seen here, a pore is hypothesized to arise due to the incorporation of tolysate $\left(\mathrm{TOS}^{-}\right.$) counterions in the polypyrrole structure. These pore structures occur randomly and are interspersed throughout the PPy membrane at intervals and frequency determined by deposition conditions. It is through these pores that charge compensating hydrated ions travel as they make their way across the gating membrane.
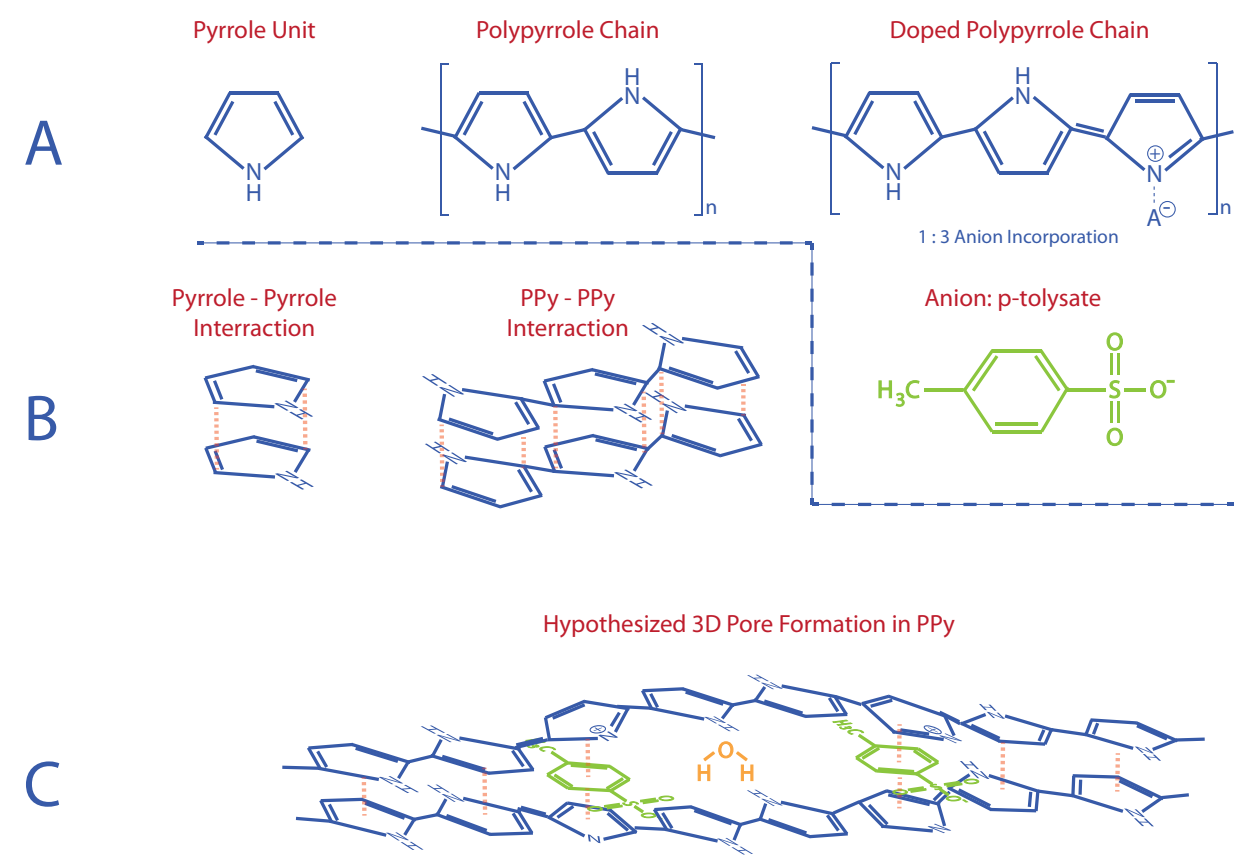

Figure 5.2: A) Primary structure representation of pyrrole, PPy chain formation, chain doping and counterion p-tolysate $\left(\mathrm{TOS}^{-}\right)$. B) Secondary structure of pyrrole-pyrrole and chain-chain interactions involving $\pi$-center co-localization. C) A tertiary structure is proposed for PPy with large immobile $\mathrm{TOS}^{-}$anions embedded, forming a pore structure in which hydrated ions may be uptaken and then released.

As membrane density and thickness increase, so do the tortuosity, mean free path and complexity of the channels ions must travel through. In the case of passive diffusion, ionic flux rates through a membrane normally decrease with increasing density and thickness. However, the opposite is true for PPy. As seen in Figure 4.17, despite $\mathrm{PPy}_{b}$ being slightly thicker and denser than $\mathrm{PPy}_{a}$, the flux of $\mathrm{KCl}_{a q}$ through this membrane is significantly higher than through $\mathrm{PPy}_{a}$, by nearly an order 
of magnitude $\left(\phi_{a}=2.15 \times 10^{-8}\right.$ moles $/ \mathrm{cm}^{2} \cdot s$ and $\left.\phi_{b}=1.12 \times 10^{-7} \mathrm{moles} / \mathrm{cm}^{2} \cdot s\right)$. These two flux rates are the average of six separate experiments each, cumulated over three different days, to assure the effects of day-to-day variability in membrane performance was taken into account. Given this precaution and the statistically different results between these membranes ( $\mathrm{p}=0.04$ by one-way ANOVA analysis), these data confirm the presence of active cycling in $\mathrm{PPy}_{\mathrm{TOS}}^{-}$membranes.

The active component of ion cycling through PPy membranes has been hypothesized by other groups (as early as 1982 by Burgmayer and Murray [77] and since [160]). However, here we show for the first time compelling data which strongly suggests active cycling predominately controls flux rates through these membranes, as opposed to being controlled by conformational changes due to PPy strand charging, as many others have hypothesized [159, 177, 178].

Despite having a slightly increased resistance to ion diffusion, the higher density membranes $\left(\mathrm{PPy}_{b}\right)$ are hypothesized to contain an increased number of binding sites available for the uptake and release of potassium ions, which exert an overwhelming influence on the flux rate of $\mathrm{KCl}_{a q}$. In fact, these data hint at complex non-linear interplay of mechanisms required to optimize ionic conductivities of the membranes. It is no longer sufficient to improve flux rates in PPy by minimizing mean free path and hydrated ion radius, as suggested by Madden (MIT Thesis [179]). There is likely an optimal membrane thickness / density combination that will provide the lowest resistance to ion movement while maximizing the number of binding sites for active pumping, which is a function of the deposition process. Indeed, two deposition schemes $\left(\mathrm{PPy}_{a}\right.$ and $\left.\mathrm{PPy}_{b}\right)$ resulted in membranes of nearly identical density and thickness, with remarkably different flux rates, which is presumably due to the difference in number of binding sites. Binding assays to test this theory and for finding the optimal combination of membrane properties is left as a consideration for future work. 


\section{$5.2 \quad H y d r o g e l$ as Artificial Intra- and Extracellular Space}

The extracellular matrix (ECM) is the space in which cells exist and permeate in vivo and in vitro. The ECM is composed mainly of collagen and glycosaminoglycans, in varying ratios depending on the cell type producing it, resulting in adaptable structural and mechanical properties. Functionally, the ECM is a space where signaling proteins, activation factors and mechanotransduction events occur to interact with cells [180]. For our purposes, however, it is considered a holding space for extracellular fluid, which is composed mainly of water and ions such as sodium and chloride (both roughly at $0.1 \mathrm{M}$ in biology).

Intracellularly, one finds the cytoplasmic matrix, which is less well known in composition than the ECM. Certainly more complex than the latter, it houses and helps partition numerous intracellular organelles. Once again, however, it may be considered more simply as a hydrated space in which ions permeate, including potassium at roughly $0.1 \mathrm{M}$ concentration.

Hydrogel provides an excellent biomimicry of these intra- and extracellular environments; it possesses a high water content, the ability to store large concentrations of ions, and a comparable polymer structure. In fact, the ECM structure is strikingly similar to hydrogels, which favors their comparison [181-184].

\subsubsection{Hydrogel Water Content}

The measured equilibrium water content (EWC) of hydrogels, as reported in Figure 4.10, offer a good indicator for the diffusivity of solutes within their structure, with diffusivity increasing with water content [124]. Hydrogels soaked in water had a

$97 \%$ water content ( $4.8 \%$ crosslinker, $\mathrm{n}=9$ ), which corresponds well to the literature for identical hydrogels [113]. The choice of initiator for the preparation of hydrogel appears to significantly affect their water content, as much lower EWC values were 
reported in identical hydrogel preparations, paired simply with different initiators $[98,185]$. With such a high level of hydration, the hydrogels produced in this lab make an excellent choice for use in the artificial cell membrane.

Lobo et al. found that the Fickian diffusion coefficient $(D)$ of $\mathrm{KCl}_{a q}$ in $1 \%$ crosslinker acrylamide hydrogels was reduced by nearly an order of magnitude as compared to solution (see Table 5.1) [98].

TABle 5.1: $\mathrm{KCl}_{a q}$ Fickian Diffusivity in Solution and Hydrogel

\begin{tabular}{c|c|c|}
\cline { 2 - 3 } Diffusivity $(D)$ & $\mathrm{KCl}_{a q}$ in Solution & $\mathrm{KCl}_{a q}$ in Hydrogel \\
\cline { 2 - 3 } $\mathrm{m}^{2} \mathrm{~s}^{-1}$ & $1.9 \times 10^{-9}$ & $8.4 \times 10^{-10}$ \\
\cline { 2 - 3 }${ }^{*}$
\end{tabular}

The reduction in ionic mobility seen in the hydrogel environmnent is caused by several factors related to the presence of a polymer network, including the molecular dynamics of the polymer chains and their interaction with the permeating solutes (ion-polymer interactions). As these ion-polymer interactions are dependent upon the chemical structures involved, diffusivity through hydrogel also varies with electrolyte composition. In fact, Lobo et al. found that the flux rate of $\mathrm{KCl}_{a q}$ through acrylamide hydrogels was significantly affected by their equilibrium water content, even more so than for a comparable salt, lithium chloride ( $\mathrm{LiCl})$ [98]. They hypothesized this was due to the water structure-breaking abilities of both chloride and potassium [186], which significantly impacted their mobility in low water content polymer environments (although their diffusivity was greater than that of $\mathrm{LiCl}$ in higher water content environments).

It is noteworthy that the water content of hydrogels exerts such a significant impact on the flux rates of $\mathrm{KCl}_{a q}$, underscoring the importance of hydrogels being manipulated and stored in an exsiccator at $100 \%$ humidity, or in solution, in order to maintain their water content prior to, and especially during, experiments. 


\subsubsection{Solute Diffusivity in Hydrogel}

In addition to water content, hydrogel pore size also significantly affects the flux rate of $\mathrm{KCl}_{a q}$ through hydrogels, as confirmed from results shown in Figure 4.20. As seen, flux rates through the PPy/hydrogel structure increase with increasing hydrogel pore size. This is caused by increasing water content, and also by changes in the conformation, microstructure and coagulation structures of the hydrogel (known as secondary, tertiary and quaternary factors) as a result of the increasing crosslinker ratios [126].

Based on Fick's First Law of diffusion (Equation 5.1) and the findings of Lobo et al. regarding the relative diffusivities of $\mathrm{KCl}_{a q}$ in solution and in hydrogel (Table 5.1), the effect of hydrogel on the flux rate through $\mathrm{PPy}_{\mathrm{TOS}}{ }^{-}$should only be approximately one order of magnitude [98]. However, as seen in Table 5.2 below, the flux rate of $\mathrm{KCl}_{a q}$ through hydrogel encapsulated $\mathrm{PPy} / \mathrm{TOS}^{-}$is two orders of magnitude lower than in solution.

A possible explanation for these decreased flux rates may be an increased charge accumulation at the surface of $\mathrm{PPy} / \mathrm{TOS}^{-}$due to the presence of the hydrogel. It is known that a double layer resistance forms on the outside of the membrane in solution, shown using Poisson-Nersnst-Planck equations to model the membrane [187]. According to Majumdar et al., it only takes a few seconds to electroneutralize these charges at the membrane in solution, allowing ionic forces to overcome the double layer resistance [187]. However, in the presence of hydrogel, we hypothesize that charges on the polymer strands, in addition to the decreased diffusivity of ions here, compound to increase the time and ionic strength necessary to overcome the double layer resistance. In addition to a longer time before flux may initiate, the hydrogel structure is likely also blocking the passage of ions by covering a portion of the surface of PPy and obstructing pore access.

Despite the significantly lower transport rates of $\mathrm{KCl}_{a q}$ through hydrogel covered $\mathrm{PPy} / \mathrm{TOS}^{-}$, these results compare favorably to biology. In fact, Piwnica-Worms et 
TABle 5.2: Flux Rate Comparison: $\mathrm{PPy}_{b}, \mathrm{PPy}_{b} /$ Hydrogel and Biology

\begin{tabular}{|c|c|c|c|}
\hline & $\mathrm{PPy}_{b}$ & $\mathrm{PPy}_{b} / \mathrm{Hydrogel}_{2 n m}$ & Transmembrane $^{a}$ \\
\hline Flux, $\phi_{K C l}\left(\right.$ moles $\left./ \mathrm{cm}^{2} \cdot s\right)$ & $1.34 \times 10^{-7}$ & $7.96 \times 10^{-10}$ & $3 \times 10^{-11}$ \\
\hline
\end{tabular}

${ }^{a}$ Transport rates for $\mathrm{Cl}^{-}$calculated across a chick cardiac cell membrane in vitro [188].

al. determined biological transmembrane flux rates of chloride to be on the order of $30 \times 10^{-12}$ moles $/ \mathrm{cm}^{2} \cdot s$ in cultured chick heart cells (see Table 5.2) [188]. Having established an order of magnitude improvement in flux rates over biological data in the $\mathrm{PPy}_{b} /$ hydrogel ${ }_{2 n m}$ structure, this work establishes an excellent system to mimic the ion gating capabilities of cell membranes in an artificial polymeric system. To the best of our knowledge, such an artificial excitable cell membrane has never been developed before, and these results present the first such prototype. 


\section{Chapter 6}

\section{Conclusions and Future Work}

The conceptual design for an autonomous artificial excitable cell analogous to the cardiac myocyte has been developed, along with a novel artificial cell membrane made from electro-active polymers which was tested for the first time. A summary of the results obtained in this work is presented below, followed by some recommendations for future work, and a brief discussion on the broader impacts of this research.

\subsection{Summary of Results}

Below is a summary of the key results characterizing both polymers, PPy and hydrogel. A quick review of the ISE experiments and their implications is then presented, followed by a recap of the main findings from the ion transport studies in both solution and hydrogel.

The research results described in this dissertation make the following contributions to the field of electro-active polymers. The first ever conceptual design for a fully synthetic heart cell was created, along with a prototype of a biomimetic cell membrane constructed from polypyrrole and hydrogel. In order to measure ionic flux rates through the artificial cell membrane, a novel electrochemical impedance spectroscopy probe was developed that is capable of electrolyte concentration measurements in 
both solution and hydrogel. This technique allowed for the measurement of the realtime concentration profile of potassium chloride in flux experiments, revealing the first ever insights into ion transport profiles within seconds after membrane opening. Furthermore, it revealed the effects of polypyrrole deposition conditions such as current density and duration on membrane structure and their effects on ion transport performance. Measured ionic flux rates in the hydrogel-PPy-hydrogel structure demonstrated successful operation as an artificial cell membrane and, additionally, were shown to surpass ionic flux rates observed in biological cardiac cell membranes. Also, first ever density measurements of PPy revealed that the assumption of 1.1 $\mathrm{g} / \mathrm{cm}^{3}$ is inaccurate, and that membrane density varies as a function of deposition conditions. Finally, an ion selective electrode detection method for potassium ions was used in a novel configuration for measuring relative potassium concentrations between two hydrogels, with an improvement in sensitivity in hydrogel over that observed in aqueous solutions.

\subsubsection{Polypyrrole and Hydrogel}

Cyclic voltammograms on PPy membranes revealed increasing hysteresis with increasing electrolyte $\mathrm{KCl}_{a q}$ concentrations. These data likely represent the formation of irreversible ion-polymer pairs in the membrane, which affect the electrochemistry and hence gating capabilities of the electroactive polymer when exposed to concentrations of $2 \mathrm{M} \mathrm{KCl}_{a q}$ and above. This was later confirmed in flux experiments, where ion transport through the membrane did not increase with increasing $\mathrm{KCl}_{a q}$ concentrations above $2 \mathrm{M}$. From these data, and the hysteresis observed in the CVs, it appears likely that the membrane's ability to capture potassium ions saturates at $1 \mathrm{M}$. It is therefore concluded that the optimal feed solution $\mathrm{KCl}_{a q}$ concentration should be no more than $1 \mathrm{M}$.

Polypyrrole membrane surfaces were also imaged via scanning electron microscopy, and shown to exhibit cauliflower-like morphologies at the electrolyte surface, with a 
smooth surface at the working electrode side. These surfaces are consistent with the literature and confirm the successful deposition of polypyrrole. Additionally, SEM images were also used to determine the actual thickness of membranes as a function of deposition conditions. These values were then compared to membrane thickness predictions of the Farrington model. Since the model overpredicted thickness values in all cases, a modification to the Farrington model is proposed, which reflects experimentally obtained membrane density measurements. In addition, the model's prediction for $y$, or the number of pyrrole units per anion, was varied to reduce the percent error of the model. It was found that the Farrington model, with such modifications, could be used to predict the thickness of membranes prepared at $2 \mathrm{~mA} / \mathrm{cm}^{2}$ with an average percent error of $3.5 \%$, as compared to $83 \%$ for those deposited at $5 \mathrm{~mA} / \mathrm{cm}^{2}$. These data imply a fundamental structural difference in membranes produced at the higher current density, which is not captured by the standard assumptions underlying the Farrington model.

Finally, equilibrium water content studies performed on hydrogels established that salt retention is occurring in their polymer matrices after soaking in various concentrations of electrolyte. This will impact the cyclical flux of $\mathrm{KCl}_{a q}$ through the proposed gel-cell and should be considered in future work, as a reduced concentration of $\mathrm{KCl}_{a q}$ will be available for transport after the initial ion-polymer binding events within both the polypyrrole and hydrogel. In addition, EIS probe calibration curves were performed in the presence of potassium chloride, in both $2 \mathrm{~nm}$ and $0.52 \mathrm{~nm}$ pore size hydrogels. These calibrations facilitated the use of EIS probes for the quantification of $\mathrm{KCl}_{a q}$ in hydrogels during flux experiments.

\subsubsection{Flux Experiments}

Ion transport studies across polypyrrole in both solution and hydrogel were performed to better understand the parameters controlling flux rates in these systems, and compare with biology. Time-resolved experiments provided valuable information 
on the flux kinetics shortly after membrane cycling. It was shown that a 100 second delay exists before the onset of ion passage into the receiving solution. This time delay corresponds to the duration of a full $\pm 1 \mathrm{~V}$ cycle at the membrane, during which the membrane is first oxidized and then reduced, which loads it with $\mathrm{K}^{+}$, ready for release with the next oxidizing potential. Theoretically, the delayed response could be diminished by the reversal of charges: by first applying a reducing potential to load the membrane with $\mathrm{K}^{+}$and then reoxidizing it to release them $(\mp 1 \mathrm{~V}$ instead of $\pm 1 \mathrm{~V}$ on the first cycle). This should cut the response delay by 50 seconds, and is left here for future consideration.

The effect of varying cycling pulse width (between 10, 30, 50, 70 and 90 seconds) on the flux rate of $\mathrm{KCl}_{a q}$ through PPy was considered. It was found that the 50 second pulse width provided a rapid flux through the membrane while also displaying a low variability between experiments. For these reasons and since cycling pulse widths in the literature are often 50 seconds as well, this pulse width was chosen as the activation standard. Furthermore, time-resolved flux experiments demonstrated significant ion transport variability from day to day. It was concluded, therefore, that experimental data should be averaged over several days to avoid observing data bias. Finally, it was also seen that PPy membranes are unable to be used for flux experiments for two consecutive days without losing significant ion transport capability, meaning that membranes should always be prepared and used for flux experiments on the same day.

Time-lapse experiments, on the other hand, conveyed oversimplified ion flux profiles across the membrane in response to cycling. It was determined that a simple correction to account for the 100 seconds delay in ion transport onset could be applied to the flux rate calculation, and this adjustment was applied to all flux rates obtained from the time-lapse data. This was possible since the transport of ions appears to be nearly linear during membrane cycling in the time-resolved $\mathrm{KCl}_{a q}$ concentration profiles obtained in solution.

Time-lapse experiments were also used to investigate the effects of membrane 
structure differences arising from different deposition schemes on ion transport rates. Two membranes, produced at $2 \mathrm{~mA} / \mathrm{cm}^{2}$ and $5 \mathrm{~mA} / \mathrm{cm}^{2}\left(\mathrm{PPy}_{b}\right.$ and $\mathrm{PPy}_{b}$, respectively), possessing nearly identical thickness and densities were compared. Despite a slightly thicker membrane and denser core (although nearly identical in value, both of which should slow diffusion through the membrane), the $\mathrm{PPy}_{b}$ membrane performed strikingly better than the other, with flux rates an order of magnitude higher than $\mathrm{PPy}_{a}$. This large discrepancy in flux rates between the two nearly identical membranes, therefore, can only be presumed due to an increased number of accessible binding sites for $\mathrm{K}^{+}$within PPy membranes formed under these conditions. Future experiments should strive, therefore, to optimize flux rates by adjusting membrane density, thickness and deposition current density. The effect of current density during deposition on binding site availability in the membrane could be confirmed via binding assays, and is left here for future consideration.

Finally, time-lapse experiments were also performed on PPy/hydrogel assemblies, which are an analog to the biological cell membrane. It was found that the pore size of hydrogel had a significant impact on the flux rates through the membrane, and highest flux rates were obtained in the largest pore size tested. Flux rates through the $\mathrm{PPy}_{b} /$ hydrogel ${ }_{2 n m}$ assembly, however, were still two orders of magnitude lower than those through $\mathrm{PPy}_{b}$ in solution. This is in part due to an order of magnitude lower diffusivity constant $(D)$ for $\mathrm{KCl}_{a q}$ in hydrogel, as compared to solution. It is also possible the reduced flux rates result from the hydrogel material covering a portion of the inlet and outlet pores in the PPy. Despite the diminished ion transport in hydrogel, average flux rates through $\mathrm{PPy}_{b} /$ hydrogel ${ }_{2 n m}$ are still an order of magnitude greater than the transmembrane flux rates of $\mathrm{Cl}^{-}$in chick cardiac cells. The artificial membrane system from $\mathrm{PPy}_{b} /$ hydrogel $_{2 n m}$, therefore, offers an excellent mimicry of biological cardiac cell membranes; both showing improved flux rates as compared to biology and responding to voltage activation in a similar way to VGICs. 


\subsubsection{Ion Selective Electrode Studies}

In order to overcome the reliance on an expensive potentiostat for the continuous monitoring of potassium concentrations in the proposed gel-cell, an inexpensive ion selective electrode (ISE) was tested in novel ways. The potentiometric response of the ISE was first measured in a standard cell environment relative to a reference electrode in buffer solution. Next, the reference electrode was replaced by an ISE, to examine the ISE vs. ISE potentiometric response in solution. This configuration produces a higher voltage drop than the ISE vs. reference electrode. The ISE vs. ISE experiments were then performed in a $\mathrm{KCl}_{a q}$ concentration gradient of $1 \mathrm{M}$ vs. 0.0001 $\mathrm{M} \mathrm{KCl}_{a q}$, to represent the ion gradients which would be present in the gel-cell. This configuration produces a lower voltage drop than the previous configuration of $1 \mathrm{M}$ vs. buffer solution, as expected. Finally, the ISEs were coated in hydrogel and tested relative to one another in the absence of the cell environment, with a $\mathrm{KCl}_{a q}$ concentration gradient of $1 \mathrm{M}$ vs. $0.00001 \mathrm{M}$ loaded in each hydrogel. The potentiometric response here is stronger than that recorded in solution. These experiments therefore demonstrate that ISEs could be used in the gel-cell as a marker for cell activation, by producing a voltage drop in response to $\mathrm{KCl}_{a q}$ concentration variations across the artificial cell membrane.

These results, therefore, represent the first example of an artificial excitable polymer cell membrane, capable of voltage-gated ion transport and high flux rates. These experiments represent the foundation for the development of the gel-cell and demonstrate concept feasibility. The work left ahead for the development of the gel-cell is briefly outlined below, with some recommendations for future work and focus. 


\subsection{Recommendations for Future Work}

\subsubsection{Excitability and Refractoriness}

Future work on this project will aim to reproduce the two key heart cell characteristics of excitability and refractoriness. This will require the use of the potassium sensing electrodes to detect the presence of the ions within the gel-cell. Initial experiments were performed to demonstrate the feasibility of these sensors in the hydrogel environment and their ability to read concentrations relative to each other. Next, it will be important to test the sensor's ability to perform across the gating membrane, and in the presence of the activating voltage placed on the latter.

The proposed ion switch, needed to make the gel-cell excitable, also remains to be produced and tested. The potassium sensors, as demonstrated in this work, establish the foundation for their successful development. It is recommended that the use of polymeric transistor logic be considered next in the production of the ion switch, such as that developed by Tybrandt [189].

Refractoriness will be imparted to the gel-cell via regions of lower salt diffusivity in hydrogel. As demonstrated in this work, the flux rates of potassium chloride are greatly affected by the porosity of hydrogel. This offers a very direct approach for the addition of a slow return pathway for ions in the gel-cell. As these modifications are made to the hydrogel material, future work should consider the material and mechanical properties of the hydrogel as well [190].

\subsubsection{Connecting Gel-Cells}

Eventually, the marker of cell activation from one gel-cell will need to be coupled to neighboring cells and used for their recruitment, in turn, to produce an excitable medium capable of propagating activation waves. The first step towards a more complex 2-D (and eventually 3-D) array will be to first develop the technology to couple two cells together, using the voltage drop produced at the lower potassium 
sensor to energize the neighboring cell's ion switch.

\subsubsection{Cell Contractility}

Recent work shows PPy membranes are capable of generating stresses larger than skeletal muscle, with strains up to $12 \%$ [191]. In addition, work done by Rajagopalan et al. reported hydrogels which respond to the presence of a critical concentration of calcium ions by contracting [192]. Harnessing these two materials and their responses in a synergistic configuration of concentric cylindrical shapes, it might be possible to achieve a gel-cell both electrically and mechanically responsive to the flux of ions. This would enable the development of a novel type of artificial muscle, with wide ranging applications from peristaltic tube pumps, to the possibility of creating artificial hearts.

\subsubsection{Component Improvement}

Since the longevity of PPy membranes is of great importance as they should be viable for extended periods of time, future work may benefit from research by Chang et al., which used high gravity PPy deposition to improve the membrane's resistance to electrochemical cycling degradation [193]. Also, to solidify the adhesion between the gating membrane and the hydrogel, a method for producing the PPy directly within the hydrogel, as shown by Kim et al., may be useful [162].

Additionally, a new method to maintain hydrogel water content will be needed for the final gel-cell design, as the flux experiments through PPy/hydrogel were performed while submersed in solution. No comparable work currently exists, but ideas include the use of sealants to encapsulate water inside the gel, such as wax, or a pore-less hydrogel mixture. 


\subsubsection{Miniaturization}

Eventually, the gel-cells will need to be produced on a microscopic scale to be interfaced by the thousands, as this project aims to produce the first of its kind synthetic cardiac tissue. Improvements in the design and assembly methods of the gel-cell will be needed, and previous work by other groups on microfabrication and micropatterning will be helpful. For example, polypyrrole membranes were successfully microfabricated by Jager et al. on a very small scale using nanolithography and etching [194]. PPy was also aligned and micro-array patterned into nanowires via electron beam lithography by another group [195]. Alternatively, a chemical PPy synthesis could be performed instead for micropatterning gel-cells on a large scale as accomplished elsewhere $[196,197]$. Additionally, an excellent method for micropatterning acrylamide hydrogels with a resolution of $2 \mu \mathrm{m}$ was demonstrated by Benedetto et al. recently [198]. These methods should be combined to facilitate the production of large arrays of nano-gel-cells. 


\section{Bibliography}

[1] Levine, H. J. Rest heart rate and life expectancy. J Am Coll Cardiol 30, 4 (1997), 11041106.

[2] Beltrami, C. A., Finato, N., Rocco, M., Feruglio, G. A., Puricelli, C., Cigola, E., Quaini, F., Sonnenblick, E. H., Olivetti, G., And Anversa, P. Structural basis of end-stage failure in ischemic cardiomyopathy in humans. Circulation 89, 1 (1994), 151163.

[3] Karafyllidis, I., And Thanailakis, A. A model for predicting forest fire spreading using cellular automata. Ecological Modelling 99, 1 (1997), 8797.

[4] Nash, M. P., And Panfilov, A. V. Electromechanical model of excitable tissue to study reentrant cardiac arrhythmias. Progress in Biophysics and Molecular Biology 85, 2-3 (2004), 501522.

[5] Haylett, K. Oesophageal Motility: A Study of the Transport Mechanism of the Oesophagus Using Fluoromanometry, Nonlinear Analysis and Oesophageal Modeling. Doctor of philosophy in medicine, University of Manchester, 2000.

[6] Aliev, R. R., Richards, W., and Wikswo, J. P. A simple nonlinear model of electrical activity in the intestine. Journal of Theoretical Biology 204, 1 (2000), 2128.

[7] Tasaki, I., Singer, I., and Takenaka, T. Effects of internal and external ionic environment on excitability of squid giant axon: A macromolecular approach. J. Gen. Physiol. 48, 6 (1965), 10951123.

[8] Hanke, W., Sieber, M., Spencer, P., Schwertner, J., And Lima, V. F. D. Properties of wave propagation in a gel-type BelousovZhabothinsky reaction under micro-gravity. Microgravity Science and Technology 21, 3 (2009), 239246 .

[9] Markus, M., And Hess, B. Isotropic cellular automaton for modelling excitable media. Nature 347, 6288 (1990), 5658.

[10] Zykov, V., And Winfree, A. Simulation of Wave Processes in Excitable Media. John Wiley \& Sons, Inc, New York, NY USA, 1992. 
[11] Greenberg, J. M., And Hastings, S. P. Spatial patterns for discrete models of diffusion in excitable media. SIAM Journal on Applied Mathematics 34, 3 (May 1978), 515523. ArticleType: primary_article / Full publication date: May, 1978 / Copyright 1978 Society for Industrial and Applied Mathematics.

[12] Winfree, A. When Time Breaks Down: The Three-Dimensional Dynamics of Electrochemical Waves and Cardiac Arrhythmias. Princeton University Press, 1987.

[13] Gerhardt, M., Schuster, H., and Tyson, J. J. A cellular automation model of excitable media including curvature and dispersion. Science 247, 4950 (1990), 15631566.

[14] Ansari, R. Polypyrrole conducting electroactive polymers: Synthesis and stability studies. E-Journal of Chemistry 3, 4 (2006), 186-201.

[15] Karyakin, A. A., Bobrova, O. A., Lukachova, L. V., and Karyakina, E. E. Potentiometric biosensors based on polyaniline semiconductor films. Sensors and Actuators B: Chemical 33, 13 (1996), 34-38.

[16] Bugar, I. Polymer structure characterization of polythiophenes by fluorescence spectroscopy in solution. Laser Physics 14, 4 (2004), 527-532.

[17] YAnG, H., AND KWAK, J. Mass transport investigated with the electrochemical and electrogravimetric impedance techniques. 1. water transport in PPy/CuPTS films. The Journal of Physical Chemistry B 101, 5 (1997), 774781.

[18] Pellegrino, J. The use of conducting polymers in membrane-based separations. Annals of the New York Academy of Sciences 984, 1 (2003), 289305.

[19] Keldermann, R., Nash, M., And Panfilov, A. Modeling cardiac mechano-electrical feedback using reaction-diffusion-mechanics systems. Physica D: Nonlinear Phenomena 238, 11-12 (2009), 10001007.

[20] Tan, H. L., Kupershmidt, S., Zhang, R., Stepanovic, S., Roden, D. M., Wilde, A. A. M., Anderson, M. E., And Balser, J. R. A calcium sensor in the sodium channel modulates cardiac excitability. Nature 415, 6870 (2002), 442447.

[21] Poole-Wilson, P. A. The dimensions of human cardiac myocytes; confusion caused by methodology and pathology. Journal of Molecular and Cellular Cardiology 27, 3 (Mar. 1995), 863865.

[22] Kohl, P., Hunter, P., And Noble, D. Stretch-induced changes in heart rate and rhythm: Clinical observations, experiments and mathematical models. Progress in Biophysics and Molecular Biology 71, 1 (1999), 91138. 
[23] Spray, D. C., And Burt, J. M. Structure-activity relations of the cardiac gap junction channel. Am J Physiol Cell Physiol 258, 2 (1990), C195C205.

[24] RoHr, S. Role of gap junctions in the propagation of the cardiac action potential. Cardiovasc Res 62, 2 (May 2004), 309322.

[25] Garces. Cell transport - biology chapter 5 presentation. http://mail.garces.org/garces1/jkloock/Prensentation\%20Chapter\%205\%2020082009_files/frame.htm, 2008.

[26] Nattel, S., Maguy, A., Bouter, S. L., And Yeh, Y.-H. Arrhythmogenic ion-channel remodeling in the heart: Heart failure, myocardial infarction, and atrial fibrillation. Physiol. Rev. 87, 2 (2007), 425456.

[27] Trautwein, W. Generation and conduction of impulses in the heart as affected by drugs. Pharmacological Reviews 15, 2 (1963), 277332.

[28] Phrommintikul, A., and Chattipakorn, N. Roles of cardiac ryanodine receptor in heart failure and sudden cardiac death. International Journal of Cardiology 112, 2 (Sept. 2006), 142152.

[29] Burgmayer, P., And Murray, R. W. Ion gate electrodes. polypyrrole as a switchable ion conductor membrane. The Journal of Physical Chemistry 88, 12 (1984), 25152521.

[30] Ehrenbeck, C., And Jttner, K. Development of an anion/cation permeable free-standing membrane based on electrochemical switching of polypyrrole. Electrochimica Acta 41, 4 (1996), 511518.

[31] Severs, N. J., Bruce, A. F., Dupont, E., And Rothery, S. Remodelling of gap junctions and connexin expression in diseased myocardium. Cardiovascular Research 80, 1 (2008), 919.

[32] Chaldoupi, S.-M., Loh, P., Hauer, R. N., Bakker, J. M. D., And Rijen, H. V. V. The role of connexin40 in atrial fibrillation. Cardiovasc Res 84, 1 (Oct. 2009), 1523.

[33] Xia, Y., Gong, K.-Z., Xu, M., Zhang, Y.-Y., Guo, J.-H., Song, Y., And ZHANG, P. Regulation of gap-junction protein connexin 43 by [beta]-adrenergic receptor stimulation in rat cardiomyocytes. Acta Pharmacol Sin 30, 7 (2009), 928-934.

[34] Gray, R. A., Pertsov, A. M., And Jalife, J. Spatial and temporal organization during cardiac fibrillation. Nature 392, 6671 (1998), 7578.

[35] QU, Z., AND WeIss, J. Dynamics and cardiac arrhythmias. Journal of Cardiovascular Electrophysiology 17, 9 (2006), 10421049. 
[36] Herlitz, J., Ekstrom, L., Wennerblom, B., Axelsson, A., Bang, A., AND HolmberG, S. Effect of bystander initiated cardiopulmonary resuscitation on ventricular fibrillation and survival after witnessed cardiac arrest outside hospital. Br Heart J 72, 5 (1994), 408412.

[37] Benjamin, E. J., Chen, P.-S., Bild, D. E., Mascette, A. M., Albert, C. M., Alonso, A., Calkins, H., Connolly, S. J., Curtis, A. B., Darbar, D., Ellinor, P. T., Go, A. S., Goldschlager, N. F., HeckBert, S. R., Jalife, J., Kerr, C. R., Levy, D., Lloyd-Jones, D. M., Massie, B. M., Nattel, S., Olgin, J. E., Packer, D. L., Po, S. S., Tsang, T. S., Wagoner, D. R. V., Waldo, A. L., and Wyse, D. G. Prevention of atrial fibrillation: Report from a national heart, lung, and blood institute workshop. Circulation 119, 4 (2009), 606618.

[38] Lloyd-Jones, D., Adams, R. J., Brown, T. M., Carnethon, M., Dai, S., Simone, G. D., Ferguson, T. B., Ford, E., Furie, K., Gillespie, C., Go, A., Greenlund, K., Haase, N., Hailpern, S., Ho, P. M., Howard, V., Kissela, B., Kittner, S., Lackland, D., Lisabeth, L., Marelli, A., McDermott, M. M., Meigs, J., Mozaffarian, D., Mussolino, M., Nichol, G., Roger, V., Rosamond, W., Sacco, R., Sorlie, P., Stafford, R., Thom, T., Wasserthiel-Smoller, S., Wong, N. D., Wylie-Rosett, J., Committee, o. B. O. T. A. H. A. S., And Subcommittee, S. S. Heart disease and stroke statistics, 2010 update: A report from the american heart association. Circulation (2009), e1170.

[39] Calkins, H., Reynolds, M. R., Spector, P., Sondhi, M., Xu, Y., Martin, A., Williams, C. J., And Sledge, I. Treatment of atrial fibrillation with anti-arrhythmic drugs or radio frequency ablation: Two systematic literature reviews and meta-analyses. Circ Arrhythm Electrophysiol (June 2009), CIRCEP.108.824789.

[40] Wijffels, M. C., Kirchhof, C. J., Dorland, R., And Allessie, M. A. Atrial fibrillation begets atrial fibrillation : A study in awake chronically instrumented goats. Circulation 92, 7 (1995), 19541968.

[41] Wijffels, M. C. E. F., Kirchhof, C. J. H. J., Dorland, R., Power, J., AND Allessie, M. A. Electrical remodeling due to atrial fibrillation in chronically instrumented conscious goats : Roles of neurohumoral changes, ischemia, atrial stretch, and high rate of electrical activation. Circulation 96, 10 (1997), 37103720.

[42] Morillo, C. A., Klein, G. J., Jones, D. L., and Guiraudon, C. M. Chronic rapid atrial pacing : Structural, functional, and electrophysiological characteristics of a new model of sustained atrial fibrillation. Circulation 91, 5 (1995), 15881595. 
[43] Gaspo, R., Bosch, R. F., Talajic, M., and Nattel, S. Functional mechanisms underlying tachycardia-induced sustained atrial fibrillation in a chronic dog model. Circulation 96, 11 (Dec. 1997), 40274035.

[44] Li, D., Fareh, S., Leung, T. K., and Nattel, S. Promotion of atrial fibrillation by heart failure in dogs : Atrial remodeling of a different sort. Circulation 100, 1 (July 1999), 8795.

[45] Pan, C.-H., Lin, J.-L., Lai, L.-P., Chen, C.-L., Huang, S. K. S., and LIN, C.-S. Downregulation of angiotensin converting enzyme II is associated with pacing-induced sustained atrial fibrillation. FEBS Letters 581, 3 (Feb. 2007), 526534 .

[46] Baxter, W. T., Mironov, S. F., Zaitsev, A. V., Jalife, J., And Pertsov, A. M. Visualizing excitation waves inside cardiac muscle using transillumination. Biophysical Journal 80, 1 (2001), 516530.

[47] Salama, G., Kanai, A., And Efimov, I. R. Subthreshold stimulation of purkinje fibers interrupts ventricular tachycardia in intact hearts: Experimental study with voltage-sensitive dyes and imaging techniques. Circ Res 74, 4 (Apr. 1994), 604619.

[48] Arora, R., Verheule, S., Scott, L., Navarrete, A., Katari, V., Wilson, E., Vaz, D., And Olgin, J. E. Arrhythmogenic substrate of the pulmonary veins assessed by high-resolution optical mapping. Circulation 10\%, 13 (Apr. 2003), 18161821.

[49] Gepstein, L., Hayam, G., And Ben-Haim, S. A. A novel method for nonfluoroscopic catheter-based electroanatomical mapping of the heart : In vitro and in vivo accuracy results. Circulation 95, 6 (1997), 16111622.

[50] Zrenner, B., Ndrepepa, G., Schneider, M., Karch, M., Hofmann, F., Schmig, A., And Schmitt, C. Computer-assisted animation of atrial tachyarrhythmias recorded with a 64-electrode basket catheter. Journal of the American College of Cardiology 34, 7 (1999), 20512060.

[51] Schmitt, C., Zrenner, B., Schneider, M., Karch, M., Ndrepepa, G., Deisenhofer, I., Weyerbrock, S., Schreieck, J., And Schomig, A. Clinical experience with a novel multielectrode basket catheter in right atrial tachycardias. Circulation 99, 18 (1999), 24142422.

[52] Pitschner, H. F., Berkovic, A., Grumbrecht, S., And Neuzner, J. Multielectrode basket catheter mapping for human atrial fibrillation. Journal of Cardiovascular Electrophysiology 9, 8 Suppl (1998), S4856. PMID: 9727676.

[53] Davidenko, J. M., Pertsov, A. V., Salomonsz, R., Baxter, W., And JALIFE, J. Stationary and drifting spiral waves of excitation in isolated cardiac muscle. Nature 355, 6358 (1992), 349351. 
[54] Baxter, W., Davidenko, J., Loew, L., Wuskell, J., and Jalife, J. Technical features of a CCD video camera system to record cardiac fluorescence data. Annals of Biomedical Engineering 25, 4 (July 1997), 713725.

[55] Kneller, J., Zou, R., Vigmond, E. J., Wang, Z., Leon, L. J., And NAtTel, S. Cholinergic atrial fibrillation in a computer model of a twodimensional sheet of canine atrial cells with realistic ionic properties. Circ Res 90, 9 (May 2002), e7387.

[56] Capelle, F. V., and Durrer, D. Computer simulation of arrhythmias in a network of coupled excitable elements. Circ Res 47, 3 (Sept. 1980), 454466.

[57] Clayton, R. H. Computational models of normal and abnormal action potential propagation in cardiac tissue: Linking experimental and clinical cardiology. Physiological Measurement 22, 3 (2001), R15R34.

[58] Tobon, C., Ruiz, C., Saiz, J., Heidenreich, E., and Hornero, F. Reentrant mechanisms triggered by ectopic activity in a three-dimensional realistic model of human atrium: A computer simulation study. In Computers in Cardiology, 2008 (2008), p. 629632.

[59] Harrild, C. S. H. . D. M. A computer model of normal conduction in the human atria. Circ Res 87, 7 (Sept. 2000), e2536.

[60] Gray, R. A., Pertsov, A. M., and Jalife, J. Incomplete reentry and epicardial breakthrough patterns during atrial fibrillation in the sheep heart. Circulation 94, 10 (Nov. 1996), 26492661.

[61] Beeler, G. W., and Reuter, H. Reconstruction of the action potential of ventricular myocardial fibres. The Journal of Physiology 268, 1 (1977), 177210. PMID: 874889.

[62] Davey, J. M., Ralph, S. F., Too, C. O., and Wallace, G. G. Synthesis, characterisation and ion transport studies on polypyrrole/polyvinylphosphate conducting polymer materials. Synthetic Metals 99, 3 (1999), 191-199.

[63] Ramachandran, V., Yoon, H., and Varadan, V. K. Potassium ion sensing with nanowire electrodes on a flexible substrate for early detection of myocardial ischemia. Journal of Nanotechnology in Engineering and Medicine 1, 1 (2010), 0110085.

[64] Zhou, D., Too, C. O., Wallace, G. G., Hodges, A. M., and Mau, A. W. H. Protein transport and separation using polypyrrole coated, platinised polyvinylidene fluoride membranes. Reactive and Functional Polymers 45, 3 (2000), 217226.

[65] Zine, N., Bausells, J., Vocanson, F., Lamartine, R., Asfari, Z., Teixidor, F., Crespo, E., Oliveira, I. M. D., SAmitier, J., And ErRACHID, A. Potassium-ion selective solid contact microelectrode based on a 
novel 1,3-(di-4-oxabutanol)-calix[4]arene-crown-5 neutral carrier. Electrochimica Acta 51, 24 (2006), 50755079.

[66] Gandhi, M. R., Murray, P., Spinks, G. M., and Wallace, G. G. Mechanism of electromechanical actuation in polypyrrole. Synthetic Metals 73, 3 (1995), 247256.

[67] Jurewicz, K., Delpeux, S., Bertagna, V., Bguin, F., And FrackOWIAK, E. Supercapacitors from nanotubes/polypyrrole composites. Chemical Physics Letters 347, 1-3 (2001), 3640.

[68] Yabuki, S., Shinohara, H., And Aizawa, M. Electro-conductive enzyme membrane. Journal of the Chemical Society. Chemical communications, 14 (1989), 945-946.

[69] Beneventi, D., Sasso, C., Zeno, E., Chaussy, D., Petit-Conil, M., AND BelgaCEM, N. Polypyrrole and polypyrole/wood-derived materials conducting composites: a review. BioResources 6, 3 (July 2011), 3585-3620.

[70] Bolto, B., And Weiss, D. Electronic conduction in polymers. II: the electrochemical reduction of polypyrrole at controlled potential. Aust. J. Chem. 16, 6 (1963), 1076-1089.

[71] Rapoport, H., and Castagnoli, N. 2,2-bipyrrole. J. Am. Chem. Soc. 84, 11 (1962), 2178-2181.

[72] Rapoport, H., Castagnoli, N., and Holden, K. G. 2,2':5',2-terpyrrole. J. Org. Chem. 29, 4 (1964), 883-885.

[73] Kanazawa, K. K., Diaz, A., Gill, W., Grant, P., Street, G., GarDINI, G. P., AND KWAK, J. Polypyrrole: An electrochemically synthesized conducting organic polymer. Synthetic Metals 1, 3 (Apr. 1980), 329336.

[74] Diaz, A., Castillo, J. I., Logan, J., And Lee, W.-Y. Electrochemistry of conducting polypyrrole films. Journal of Electroanalytical Chemistry and Interfacial Electrochemistry 129, 1-2 (1981), 115132.

[75] Gerard, M., Chaubey, A., and Malhotra, B. Application of conducting polymers to biosensors. Biosensors and Bioelectronics 17, 5 (2002), 345-359.

[76] Bloor, D., And Movaghar, B. Conducting polymers. Solid-State and Electron Devices, IEE Proceedings I 130, 5 (1983), 225-232.

[77] Burgmayer, P., And Murray, R. W. An ion gate membrane: Electrochemical control of ion permeability through a membrane with an embedded electrode. Journal of the American Chemical Society 104, 22 (1982), 61396140.

[78] Zhao, H., Price, W., Too, C., Wallace, G., and Zhou, D. Parameters influencing transport across conducting electroactive polymer membranes. Journal of Membrane Science 119, 2 (1996), 199-212. 
[79] Moavenian, M., Torabi, K., And Tahani, M. Analytical dynamic modeling of fast trilayer polypyrrole bending actuators. Smart Materials and Structures 20, 11 (2011), 115020-115028.

[80] Han, G., AND SHI, G. Electrochemical actuator based on single-layer polypyrrole film. Sensors and Actuators B: Chemical 113, 1 (2006), 259264.

[81] Madden, J. D. Polypyrrole actuators: Properties and initial applications. In Electroactive Polymers for Robotic Applications. Springer London, 2007, pp. 121-152.

[82] Alici, G., Metz, P., And Spinks, G. M. A methodology towards geometry optimization of high performance polypyrrole (PPy) actuators. Smart Materials and Structures 15, 2 (2006), 243252.

[83] Deslouis, C., Moustafid, T. E., Musiani, M. M., and Tribollet, B. Mixed ionic-electronic conduction of a conducting polymer film: AC impedance study of polypyrrole. Electrochimica Acta 41, 7-8 (1996), 13431349.

[84] WEI, D. Dye sensitized solar cells. International Journal of Molecular Sciences 11 (2010), 1103-1113.

[85] Cui, L., Shen, J., Cheng, F., Tao, Z., And Chen, J. SnO2 nanoparticlespolypyrrole nanowires composite as anode materials for rechargeable lithium-ion batteries. Journal of Power Sources 196, 4 (2011), 2195-2201.

[86] Kaneto, K., Yoshino, K., And Inuishi, Y. Characteristics of electrooptic device using conducting polymers, polythiophene and polypyrrole films. Japanese Journal of Applied Physics 22 (1983), L412-L414.

[87] Girotto, E. M., And De Paoli, M. Polypyrrole color modulation and electrochromic contrast enhancement by doping with a dye. Advanced Materials 10, 10 (1998), 790-793.

[88] Beaujuge, P. M., And Reynolds, J. R. Color control in -conjugated organic polymers for use in electrochromic devices. Chem. Rev. 110, 1 (2010), 268-320.

[89] HuAng, Y. M. Electroluminescence of naturally oxidized polypyrrole. Solid State Phenomena 181-182 (2011), 245-250.

[90] Ponce de Leon, C., Campbell, S. A., Smith, J. R., and Walsh, F. C. Conducting polymer coatings in electrochemical technology part 2 application areas. Transactions of the Institute of Metal Finishing 86, 1 (2008), 34-40.

[91] Ateh, D., Vadgama, P., And Navsaria, H. Culture of human keratinocytes on polypyrrole-based conducting polymers. Tissue Engineering 12, 4 (2006), 645-655. 
[92] Silk, T., Hong, Q., Tamm, J., and Compton, R. G. AFM studies of polypyrrole film surface morphology i: The influence of film thickness and dopant nature. Synthetic Metals 93, 1 (1998), 5964.

[93] Davey, J. M., Ralph, S. F., Too, C. O., Wallace, G. G., and ParTRIDGE, A. C. Electrochemically controlled transport of metal ions across polypyrrole membranes using a flow-through cell. Reactive and Functional Polymers 49, 2 (2001), 8798.

[94] Pedley, D. G., Skelly, P. J., and Tighe, B. J. Hydrogels in biomedical applications. British Polymer Journal 12, 3 (1980), 99-110.

[95] Ramaraj, B., and Radhakrishnan, G. Modification of the dynamic swelling behaviour of poly(2-hydroxyethyl methacrylate) hydrogels in water through interpenetrating polymer networks (IPNs). Polymer 35, 10 (May 1994), 2167-2173.

[96] Gehrke, S., And LeE, P. Hydrogels for drug delivery systems. Journal of pharmaceutical sciences 41 (1990), 333-85.

[97] Wichterle, O., And Lim, D. Hydrophilic gels for biological use. Nature 185, 4706 (1960), 117-118.

[98] Lobo, V., Valente, A., Polishchuk, A., and Geuskens, G. Transport of non-associated electrolytes in acrylamide hydrogels. Journal of Molecular Liquids 94, 3 (2001), 179-192.

[99] Derrico, G., De lellis, M., Mangiapia, G., Tedeschi, A., Ortona, O., Fusco, S., Borzacchiello, A., and Ambrosio, L. Structural and mechanical properties of UV-photo-cross-linked poly(N-vinyl-2-pyrrolidone) hydrogels. Biomacromolecules 9, 1 (2007), 231-240.

[100] Zhang, S. D., Zhai, Y. C., And Zhang, Z. F. Preparation and properties of polyvinyl alcohol (PVA)/Polyvinyl pyrrolidone (PVP) hydrogel. Applied Mechanics and Materials 84-85 (2011), 485-488.

[101] Schmedlen, R. H., Masters, K. S., and West, J. L. Photocrosslinkable polyvinyl alcohol hydrogels that can be modified with cell adhesion peptides for use in tissue engineering. Biomaterials 23, 22 (2002), 4325-4332.

[102] Mudassir, J., And Ranjha, N. M. Dynamic and equilibrium swelling studies: crosslinked $\mathrm{pH}$ sensitive methyl methacrylate-co-itaconic acid (MMA-coIA) hydrogels. Journal of Polymer Research 15 (2007), 195-203.

[103] Leahy, C., Mandell, R., and Lin, S. Initial in vivo tear protein deposition on individual hydrogel contact lenses. Optometry $\& 3$ Vision Science 67, 7 (1990), 504-511. 
[104] Kazanskit, K., And Dubrovskit, S. Chemistry and physics of "agricultural" hydrogels. Advances in Polymer Science 104 (1992), 97-133.

[105] Watanabe, J., And Akashi, M. Novel biomineralization for hydrogels: electrophoresis approach accelerates hydroxyapatite formation in hydrogels. Biomacromolecules 7, 11 (2006), 3008-3011.

[106] Beebe, D. J., Moore, J. S., Bauer, J. M., Yu, Q., Liu, R. H., DevaDoss, C., AND Jo, B.-H. Functional hydrogel structures for autonomous flow control inside microfluidic channels. Nature 404, 6778 (2000), 588-590.

[107] Kesting, R. E. Synthetic Polymeric Membranes: A Structural Perspective, 2nd ed. Wiley-Interscience, 1985.

[108] Mulder, M. Basic Principles of Membrane Technology Second Edition. Springer, 1996.

[109] Klabunde, K. J., And Richards, R. M. Nanoscale Materials in Chemistry. John Wiley \& Sons, 2009.

[110] Ishikiriyama, K., Todoki, M., and Motomura, K. Pore size distribution (PSD) measurements of silica gels by means of differential scanning calorimetry: I. optimization for determination of PSD. Journal of Colloid and Interface Science 171, 1 (1995), 92-102.

[111] Glaves, C. L., And Smith, D. M. Membrane pore structure analysis via NMR spinlattice relaxation experiments. Journal of Membrane Science 46, 23 (1989), 167-184.

[112] Gagnon, M.-A. Etude des Proprietes de Transport dans les Hydrogels de Curdlan. These ou memoire numerique / electronic thesis or dissertation, Universite de Montreal, 2009.

[113] Lira, L. M., Martins, K. A., And Torresi, S. I. C. D. Structural parameters of polyacrylamide hydrogels obtained by the equilibrium swelling theory. European Polymer Journal 45, 4 (2009), 1232-1238.

[114] Barthus, R. C., Lira, L. M., And Torresi, S. I. C. D. Conducting polymer- hydrogel blends for electrochemically controlled drug release devices. Journal of the Brazilian Chemical Society 19, 4 (2008), 630-636. FAPESP.

[115] Eddington, D. T., And Beebe, D. J. Flow control with hydrogels. Advanced Drug Delivery Reviews 56, 2 (2004), 199-210.

[116] Tanaka, T., Nishio, I., Sun, S.-T., And Ueno-Nishio, S. Collapse of gels in an electric field. Science 218, 4571 (1982), $467-469$.

[117] Tanaka, T., Fillmore, D., Sun, S.-T., Nishio, I., Swislow, G., And Shah, A. Phase transitions in ionic gels. Physical Review Letters 45, 20 (1980), 1636-1639. 
[118] Hoffmann, J., Pltner, M., Kuckling, D., And Fischer, W.-J. Photopatterning of thermally sensitive hydrogels useful for microactuators. Sensors and Actuators A: Physical 77, 2 (1999), 139-144.

[119] Suzuki, A., And TAnakA, T. Phase transition in polymer gels induced by visible light. Nature 346, 6282 (1990), 345-347.

[120] Kato, N., Yamanobe, S., And Takahashi, F. Property of magnetodriven poly (N-Isopropylacrylamide) gel containing -Fe2O3 in $\mathrm{NaCl}$ solution as a chemomechanical device. Materials Science and Engineering: C 5, 2 (1997), $141-147$.

[121] Kuhn, W., Hargitay, B., Katchalsky, A., And Eisenberg, H. Reversible dilation and contraction by changing the state of ionization of highpolymer acid networks. Nature 165, 4196 (1950), 514-516.

[122] Horkay, F., TAsaki, I., And Basser, P. J. Osmotic swelling of polyacrylate hydrogels in physiological salt solutions. Biomacromolecules 1, 1 (2000), 84-90.

[123] Lobo, V. M., And Valente, A. J. A capillary cell for measuring diffusion coefficients of electrolyte solutions in polymers. Polymer Degradation and Stability 44, 2 (1994), 147-150.

[124] Wu, Y., Joseph, S., And Aluru, N. R. Effect of cross-linking on the diffusion of water, ions, and small molecules in hydrogels. J. Phys. Chem. B 113, 11 (2009), 3512-3520.

[125] YezeK, L. P., And van Leeuwen, H. P. Donnan effects in the steady-state diffusion of metal ions through charged thin films. Langmuir 21, 23 (2005), $10342-10347$.

[126] SAKai, K. Determination of pore size and pore size distribution: 2. dialysis membranes. Journal of Membrane Science 96, 1-2 (1994), 91-130.

[127] Pappenheimer, J. R., Renkin, E. M., and Borrero, L. M. Filtration, diffusion and molecular sieving through peripheral capillary membranes: A contribution to the pore theory of capillary permeability. Am J Physiol 167, 1 (1951), 13-46.

[128] Verniory, A., Du Bois, R., Decoodt, P., Gassee, J. P., And Lambert, P. P. Measurement of the permeability of biological membranes application to the glomerular wall. The Journal of General Physiology 62, 4 (1973), 489 -507.

[129] Yasuda, H., Lamaze, C. E., And Ikenberry, L. D. Permeability of solutes through hydrated polymer membranes. part i: Diffusion of sodium chloride. Die Makromolekulare Chemie 118, 1 (1968), 19-35. 
[130] Zhang, S., Zhai, Y., And Zhang, Z. Study on medical polyvinyl alcohol (PVA) / polyvinyl pyrrolidone (PVP) hydrogel burns dressing. In 2011 International Conference on Electronic and Mechanical Engineering and Information Technology (EMEIT) (Aug. 2011), vol. 9, IEEE, p. 44854488.

[131] Mathews, D. T., Birney, Y. A., Cahill, P. A., And McGuinness, G. B. Vascular cell viability on polyvinyl alcohol hydrogels modified with WaterSoluble and Insoluble chitosan. Journal of Biomedical Materials Research Part B: Applied Biomaterials 84B, 2 (2008), 531-540.

[132] Li, F., Su, Y., Wang, J., Wu, G., And Wang, C. Influence of dynamic load on friction behavior of human articular cartilage, stainless steel and polyvinyl alcohol hydrogel as artificial cartilage. Journal of Materials Science: Materials in Medicine 21 (2009), 147-154.

[133] Li, F., Su, Y. L., Shi, D. F., And Wang, C. T. Comparison of human articular cartilage and polyvinyl alcohol hydrogel as artificial cartilage in microstructure analysis and unconfined compression. Advanced Materials Research 87-88 (2009), 188-193.

[134] Babu, D. M., C, S. S., Shah, A. P., And H G, S. Preparation and characterization of poly(2-hydroxyethyl methacrylateco- methyl methacrylate) hydrogels for sustained delivery of antitumor drug. Current Drug Delivery (2011). PMID: 22023209.

[135] Ikariyama, Y., And Heineman, W. R. Polypyrrole electrode as a detector for electroinactive anions by flow injection analysis. Anal. Chem. 58, 8 (1986), 1803-1806.

[136] Ikariyama, Y., Galiatsatos, C., Heineman, W. R., and Yamauchi, S. Polypyrrole electrode as a detector of anionic substances. Sensors and Actuators 12, 4 (1987), 455-461.

[137] Momma, T., Komaba, S., Yamamoto, M., Osaka, T., and Yamauchi, S. All-solid-state potassium-selective electrode using double-layer film of polypyrrole/polyanion composite and plasticized poly(vinyl chloride) containing valinomycin. Sensors and Actuators B: Chemical 25, 13 (1995), 724-728.

[138] Pandey, P. C., Singh, G., And Srivastava, P. K. Electrochemical synthesis of tetraphenylborate doped polypyrrole and its applications in designing a novel zinc and potassium ion sensor. Electroanalysis 14, 6 (2002), 427432.

[139] Momma, T., Yamamoto, M., Komaba, S., and Osaka, T. Analysis of the long-term potential stability of an all-solid-state potassium-selective electrode with electroactive polypyrrole film. Journal of Electroanalytical Chemistry 40\%, 12 (1996), 91-96. 
[140] Chang, T. M. S. Artificial Cells: Biotechnology, Nanomedicine, Regenerative Medicine, Blood Substitutes, Bioencapsulation, and Cell/Stem Cell Therapy. World Scientific, 2007.

[141] Zhou, D., Spinks, G. M., Wallace, G. G., Tiyapiboonchaiya, C., MacFarlane, D. R., Forsyth, M., And Sun, J. Solid state actuators based on polypyrrole and polymer-in-ionic liquid electrolytes. Electrochimica Acta 48, 14-16 (June 2003), 23552359.

[142] Mukai, K., Asaka, K., Kiyohara, K., Sugino, T., Takeuchi, I., Fukushima, T., And AidA, T. High performance fully plastic actuator based on ionic-liquid-based bucky gel. Electrochimica Acta 53, 17 (2008), 55555562.

[143] Richter, A. Hydrogels for actuators. In Hydrogel Sensors and Actuators, G. Gerlach and K.-F. Arndt, Eds., vol. 6. Springer Berlin Heidelberg, Berlin, Heidelberg, 2009, pp. 221-248.

[144] Hu, Z., Baralia, G., Bayot, V., Gohy, J.-F., And Jonas, A. M. Nanoscale control of polymer crystallization by nanoimprint lithography. Nano Letters 5, 9 (2005), 17381743.

[145] Wallace, G. G., Spinks, G. M., Kane-Maguire, L. A. P., and TeasDALE, P. R. Conductive Electroactive Polymers: Intelligent Polymer Systems, third edition ed. CRC Press, Taylor \& Francis Group, Boca Raton, FL, 2009.

[146] Zhao, H., Price, W., And Wallace, G. Electrochemically controlled transport of potassium chloride across a conducting electro-active polymer membrane. Journal of Electroanalytical Chemistry 334, 1-2 (1992), 111-120.

[147] Tietje-Girault, J., Ponce De Len, C. A., and Walsh, F. C. Electrochemically deposited polypyrrole films and their characterization. Surface and Coatings Technology 201, 12 (2007), 6025-6034.

[148] Farrington, A. M., And Slater, J. M. Prediction and characterization of the charge/size exclusion properties of overoxidized poly(pyrrole) films. Electroanalysis 9, 11 (1997), 843-847.

[149] East, G. A., And Valle, M. A. D. Easy-to-make Ag/AgCl reference electrode. Journal of Chemical Education 7\%, 1 (2000), 97.

[150] Mirmohseni, A., Price, W., And Wallace, G. Electrochemically controlled transport of small charged organic molecules across conducting polymer membranes. Journal of Membrane Science 100, 3 (1995), 239-248.

[151] Partridge, A. C., Milestone, C. B., Too, C. O., and Wallace, G. G. Polypyrrole based cation transport membranes. Journal of Membrane Science 152, 1 (1999), 6170. 
[152] Wang, E., Liu, Y., Samec, Z., And Dvok, C. Charge transfer across a conducting polypyrrole membrane separated by two electrolyte solutions. Electroanalysis 2, 8 (1990), 623629.

[153] Iseki, M., Saito, K., Ikematsu, M., Sugiyama, Y., Kuhara, K., AND MizukAmi, A. Effect of cations on the electrochemical behavior of ptoluenesulfonate-doped polypyrrole in various aqueous solutions. Journal of Electroanalytical Chemistry 358, 12 (1993), 221-233.

[154] Akieh, M. N., Varga, ., Latonen, R.-M., Ralph, S. F., Bobacka, J., AND IVAskA, A. Simultaneous monitoring of the transport of anions and cations across polypyrrole based composite membranes. Electrochimica Acta 56, 10 (2011), 3507-3515.

[155] Weidlich, C., Mangold, K.-M., And Jttner, K. EQCM study of the ion exchange behaviour of polypyrrole with different counterions in different electrolytes. Electrochimica Acta 50, 78 (2005), 1547-1552.

[156] Dusemund, C., And Schwitzgebel, G. Ion-exchange properties of conducting polypyrrole films. Synthetic Metals 55, 23 (1993), 1396-1401.

[157] Muramatsu, H., Ye, X., Suda, M., Sakuhara, T., and Ataka, T. In-situ monitoring of microrheology on electrochemical deposition using an advanced quartz crystal analyzer and its application to polypyrrole deposition. Journal of Electroanalytical Chemistry 322, 12 (1992), 311-323.

[158] Majumdar, S., Kargupta, K., Ganguly, S., and Ray, P. Studies on the performance of the conducting polymer based molecular release system. Polymer Engineering \& Science 51, 10 (Oct. 2011), 2001-2012.

[159] Wang, E., Liu, Y., Dong, S., And Ding, J. Electrochemical study of ion transfer across the polypyrrole membrane between two electrolyte solutions. Journal of the Chemical Society, Faraday Transactions 86, 12 (1990), 2243.

[160] Akieh, M. N., Ralph, S. F., Bobacka, J., And Ivaska, A. Transport of metal ions across an electrically switchable cation exchange membrane based on polypyrrole doped with a sulfonated calix[6]arene. Journal of Membrane Science 354, 12 (2010), 162-170.

[161] Pernaut, J.-M., And Reynolds, J. R. Use of conducting electroactive polymers for drug delivery and sensing of bioactive molecules. a redox chemistry approach. The Journal of Physical Chemistry B 104, 17 (May 2000), 4080-4090.

[162] Kim, B., Spinks, G., Wallace, G., And John, R. Electroformation of conducting polymers in a hydrogel support matrix. Polymer 41, 5 (Mar. 2000), 1783-1790. 
[163] Kagz, H., zGM, S., And Orbay, M. Preparation of modified polyacrylamide hydrogels and application in removal of $\mathrm{Cu}(\mathrm{II})$ ion. Polymer 42, 18 (2001), 7497-7502.

[164] Baker, C. K., Qiu, Y. J., And Reynolds, J. R. Electrochemically-induced charge and mass transport in polypyrrole/poly(styrene sulfonate) molecular composites. The Journal of Physical Chemistry 95, 11 (1991), 4446-4452.

[165] Ibanez, J. G., Alatorre-Ordaz, A., Gutierrez-Granados, S., And Batina, N. Nanoscale degradation of polypyrrole films under oxidative stress: An atomic force microscopy study and review. Polymer Degradation and Stability 93, 4 (2008), 827-837.

[166] Price, A. D., Gillen, T., Liu, C. C., O’Shaughnessy, C. A., And NAguib, H. E. Evaluation of porous membrane core elasticity and porous morphology for polypyrrole trilayer actuators. Journal of Cellular Plastics 48 , 1 (Jan. 2012), 25-42.

[167] Sixou, B., Mermilliod, N., And Travers, J. P. Aging effects on the transport properties in conducting polymer polypyrrole. Physical Review B 53, 8 (1996), 4509-4521.

[168] Cheung, K., Bloor, D., And Stevens, G. Characterization of polypyrrole electropolymerized on different electrodes. Polymer 29, 9 (1988), 1709-1717.

[169] Siwy, Z., Gu, Y., Spohr, H. A., Baur, D., Wolf-Reber, A., Spohr, R., Apel, P., And Korchev, Y. E. Rectification and voltage gating of ion currents in a nanofabricated pore. Europhysics Letters (EPL) 60, 3 (Nov. 2002), 349-355.

[170] Hayden, O., And Nielsch, K. Molecular- and Nano-Tubes. Springer, 2011.

[171] Jovanovic-Talisman, T., Tetenbaum-Novatt, J., McKenney, A. S., Zilman, A., Peters, R., Rout, M. P., And Chait, B. T. Artificial nanopores that mimic the transport selectivity of the nuclear pore complex. Nature 45\%, 7232 (2008), 1023-1027.

[172] Saleh, O. A., And Sohn, L. L. An artificial nanopore for molecular sensing. Nano Letters 3, 1 (2003), 37-38.

[173] Zhu, B., Li, J., Chen, Q., CaO, R.-G., Li, J., And Xu, D. Artificial, switchable $\mathrm{k}+$-gated ion channels based on flow-through titania-nanotube arrays. Physical chemistry chemical physics: PCCP 12, 34 (2010), 9989-9992. PMID: 20725665.

[174] Sexton, L. T., Horne, L. P., and Martin, C. R. Developing synthetic conical nanopores for biosensing applications. Molecular bioSystems 3, 10 (2007), 667-685. PMID: 17882330. 
[175] Majumder, M., Zhan, X., Andrews, R., And Hinds, B. J. Voltage gated carbon nanotube membranes. Langmuir 23, 16 (2007), 8624-8631.

[176] Ariza, M. J., And Otero, T. F. Nitrate and chloride transport through a smart membrane. Journal of Membrane Science 290, 12 (2007), 241-249.

[177] Otero, T. F., Grande, H., and Rodrguez, J. Role of conformational relaxation on the voltammetric behavior of polypyrrole. experiments and mathematical model. The Journal of Physical Chemistry B 101, 42 (1997), 85258533 .

[178] Ren, X., And Pickup, P. G. Impedance measurements of ionic conductivity as a probe of structure in electrochemically deposited polypyrrole films. Journal of Electroanalytical Chemistry 396, 12 (1995), 359-364.

[179] Madden, J. D. W. Conducting Polymer Actuators. Thesis, Massachusetts Institute of Technology, 2000. Thesis (Ph.D.)-Massachusetts Institute of Technology, Dept. of Mechanical Engineering, 2000.

[180] Badylak, S. F., Freytes, D. O., and Gilbert, T. W. Extracellular matrix as a biological scaffold material: Structure and function. Acta biomaterialia 5, 1 (2009), 1-13. PMID: 18938117.

[181] Kapellos, G. E., Alexiou, T. S., and Payatakes, A. C. A multiscale theoretical model for diffusive mass transfer in cellular biological media. Mathematical Biosciences 210, 1 (2007), 177-237.

[182] Rowley, J. A., Madlambayan, G., And Mooney, D. J. Alginate hydrogels as synthetic extracellular matrix materials. Biomaterials 20, 1 (1999), $45-53$.

[183] Shu, X. Z., Liu, Y., Palumbo, F., and Prestwich, G. D. Disulfidecrosslinked hyaluronan-gelatin hydrogel films: a covalent mimic of the extracellular matrix for in vitro cell growth. Biomaterials 24, 21 (2003), 3825-3834.

[184] Tibbitt, M. W., And Anseth, K. S. Hydrogels as extracellular matrix mimics for 3D cell culture. Biotechnology and Bioengineering 103, 4 (2009), 655-663.

[185] Ferreira, L., Vidal, M. M., And Gil, M. H. Design of a drug-delivery system based on polyacrylamide hydrogels. evaluation of structural properties. The Chemical Educator 6, 2 (2001), 100-103.

[186] Marcus, Y. Effect of ions on the structure of water. Pure and Applied Chemistry 82, 10 (2010), 1889-1899.

[187] Majumdar, S., Ray, P. S., Kargupta, K., And Ganguly, S. Ionic ingress and charge neutralization phenomena of conducting polymer films. ChemPhysChem 11, 1 (Jan. 2010), 211-219. 
[188] Piwnica-Worms, D., Jacob, R., Horres, C. R., and Lieberman, M. Transmembrane chloride flux in tissue-cultured chick heart cells. The Journal of General Physiology 81, 5 (1983), 731-748.

[189] Tybrandt, K. Ionic Circuits for Transduction of Electronic Signals into Biological Stimuli. Doctoral thesis, Linkping University, The Institute of Technology, Linkping, Sweden, 2012.

[190] Dai, T., Qing, X., Lu, Y., And Xia, Y. Conducting hydrogels with enhanced mechanical strength. Polymer 50, 22 (2009), 5236-5241.

[191] Paster, E., Ruddy, B., Pillai, P., And Hunter, I. Closed loop performance of polypyrrole linear contractile actuators. pp. $506-511$.

[192] Rajagopalan, S., Feldman, A., and Fernandes, J. Designing artificial muscles: A biomimetic approach. In Proceedings of the Canadian Engineering Education Association (McGill University, Montreal, QC, July 2004).

[193] Chang, J. H., Aleman de Leon, C. R., and Hunter, I. W. Selfassembled, nanostructured polypyrrole films grown in a high-gravity environment. Langmuir (2012).

[194] Jager, E. W. H., Smela, E., And Ingans, O. Microfabricating conjugated polymer actuators. Science 290, 5496 (2000), 1540 -1545.

[195] Xia, L., Quan, B., And Wei, Z. Patterned growth of vertically aligned polypyrrole nanowire arrays. Macromolecular Rapid Communications In Press (2011).

[196] Green, R. A., Baek, S., Poole-Warren, L. A., and Martens, P. J. Conducting polymer-hydrogels for medical electrode applications. Science and Technology of Advanced Materials 11, 1 (2010), 014107.

[197] Gomez, N., Lee, J. ., Nickels, J. ., And Schmidt, C. . Micropatterned polypyrrole: A combination of electrical and topographical characteristics for the stimulation of cells. Advanced Functional Materials 17, 10 (2007), 16451653 .

[198] Benedetto, F. D., Biasco, A., Pisignano, D., and Cingolani, R. Patterning polyacrylamide hydrogels by soft lithography. Nanotechnology 16, 5 (May 2005), S165-S170.

[199] Capiglia, C., Saito, Y., Yamamoto, H., Kageyama, H., and MustarelLi, P. Transport properties and microstructure of gel polymer electrolytes. Electrochimica Acta 45, 8-9 (Jan. 2000), 1341-1345.

[200] Fuller, J., Breda, A., And Carlin, R. Ionic liquid-polymer gel electrolytes from hydrophilic and hydrophobic ionic liquids. Journal of Electroanalytical Chemistry 459, 1 (1998), 29-34. 
[201] Yeon, S.-H., Kim, K.-S., Choi, S., Cha, J.-H., and Lee, H. Characterization of PVdF(HFP) gel electrolytes based on 1-(2-hydroxyethyl)-3-methyl imidazolium ionic liquids. The Journal of Physical Chemistry B 109, 38 (2005), 1792817935 .

[202] Fukushima, T., Asaka, K., Kosaka, A., And Aida, T. Fully plastic actuator through layer-by-layer casting with ionic-liquid-based bucky gel. Angewandte Chemie International Edition 44, 16 (2005), 24102413.

[203] Giroud, N., Rouault, H., Chainet, E., and Poignet, J.-C. Properties of BMIBF4-LiBF4 electrolytes for lithium ion batteries. In Ionic Liquid Electrolytes (Honolulu, HI, 2009), vol. 16 (35), ECS, pp. 75-88. 


\section{Appendix A}

\section{Summary of Failed Experiments}

\section{A.1 Ionic Liquid Polymer Gels}

Ionic liquid polymer gels (ILPG) are composed of an ionic liquid (i.e. a molten salt) combined with a polymer scaffold to form a highly porous semi-solid material in which ions permeate freely [199]. It is their ability to integrate mobile ions that initially made them an attractive candidate for the gel-cell.

The mixture of ionic liquid with PVDF according to a recipe by Fuller et al. [200] creates a network of continuous channels within the gel, forming a solid state ionic liquid with very high specific ionic conductivity, on the order of $10^{-3} \mathrm{~S} / \mathrm{cm}$ [201]. The consistency of the ILPG is similar to that of a very soft rubber, with a Young's Modulus around $70 \mathrm{MPa}$ [142]. Given the pliability and high ionic mobility in ILPGs, ion movement within the material has been harnessed to produce deformation. Fukushima et al. [202], for example, were able to induce a shrink / swell response in the ILPG by the application of an electrical field to a disproportionately sized ion pair, driving small anions and large cations within the material towards the anode and cathode, respectively (Figure A.1).

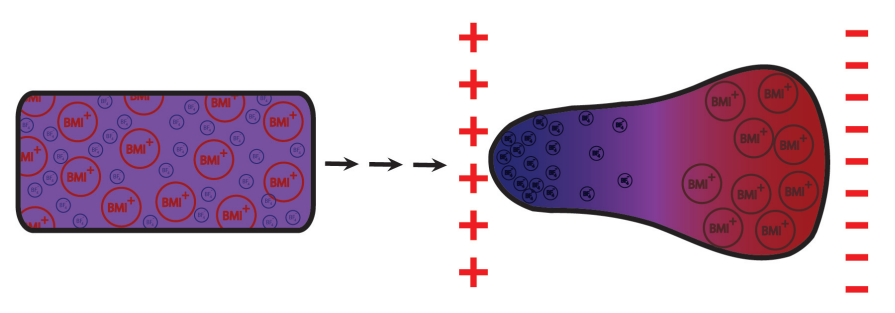

Figure A.1: Schematic representation of ion redistribution and deformation produced in the ILPG, in response to an external electrical field. Left: Undeformed gel with random distribution of ions $\mathrm{BMIm}^{+}$and $\mathrm{BF}_{4}^{-}$-. Right: A gradient in ion distribution is seen: large cations accumulate and swell at the cathode, and smaller anions accumulate at the anode. Fluid entrainement brings liquid in the gel away from the anode and towards to cathode.

Fuller et al. found that the ionic conductivity of ILPG could be changed merely by altering the polymer to ionic liquid ratio (see Figure A.2) [200]. The idea of shrinking 
material pore size to control the flux of ions was ultimately used in the gel-cell with hydrogels instead, in order to fine-tune the refractory period length via the return valve.

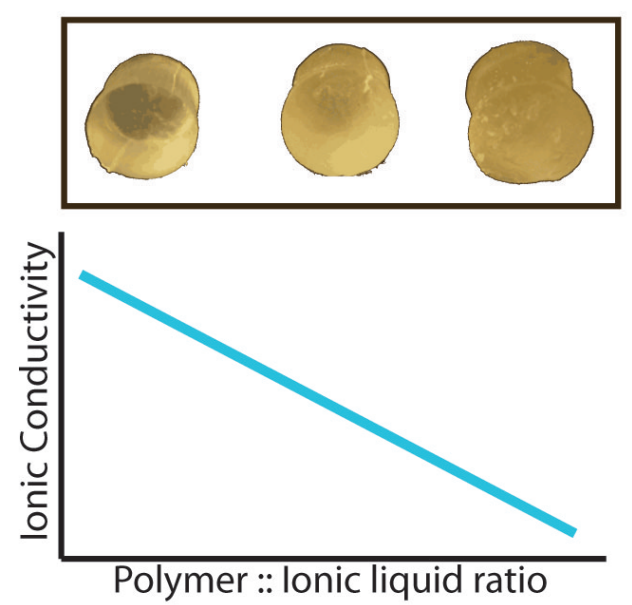

Figure A.2: Top: Ionic liquid polymer gels created in the Soft Materials Lab at UVa, with increasing ratios of polymer to ionic liquid from left to right. Visible material property changes, including shape integrity and material opacity along with an increase in Youngs modulus. Graph: Increasing the polymer to ionic liquid ratio in the ILPG results in reduced ionic conductivity of the gel.

For use in the gel-cell, potassium chloride salts were added to the ILPG preparation at varying ratios. Initially incorporated at $16.67 \mathrm{wt} \%, \mathrm{KCl}$ remained as a sediment instead of dissolving into the mixture. Its concentration in the ILPG was therefore reduced until a gel was produced with fully dissolved $\mathrm{KCl}$. The final recipe for the KCl-containing ILPG consisted of 2.5 wt \% KCl, 33 wt \% PVDF(HFP) and 65 wt $\% \mathrm{BMiBF}_{4}$, and was produced as described above.

\section{A.1.1 Mass Spectroscopy}

Mass spectrometry (a method used to determine the elemental composition of a sample) was performed on samples of $1 \mu \mathrm{M}, 100 \mu \mathrm{M}, 10 \mathrm{mM}$ and $1 \mathrm{M} \mathrm{KCl}_{a q}$, along with samples of ionic liquid, and ionic liquid saturated with $\mathrm{KCl}$. Erin Jeffre from the Mass Spec Center at the University of Virginia ran these tests. Unfortunately, the presence of $\mathrm{KCl}$ was not detected in the samples, likely because of the volatilization temperature of $\mathrm{KCl}$ was much higher than the capacity of the spectrometer available. Another technique, electrospraying the samples, was then attempted. This technique also revealed no presence of $\mathrm{KCl}$ in the samples.

\section{A.1.2 Ultraviolet-Visible Spectroscopy}

Spectroscopy in the ultraviolet range (UV-Vis) was then performed on the same samples as above to detect the presence of $\mathrm{KCl}$ between 190 and $700 \mathrm{~nm}$. However, the wavelenthgs of importance for $\mathrm{KCl}$ were within the bandwidth of a large response 
for the ionic liquid, overshadowing the presence of $\mathrm{KCl}$ in the sample, invalidating this detection method as well (see Figure A.3).

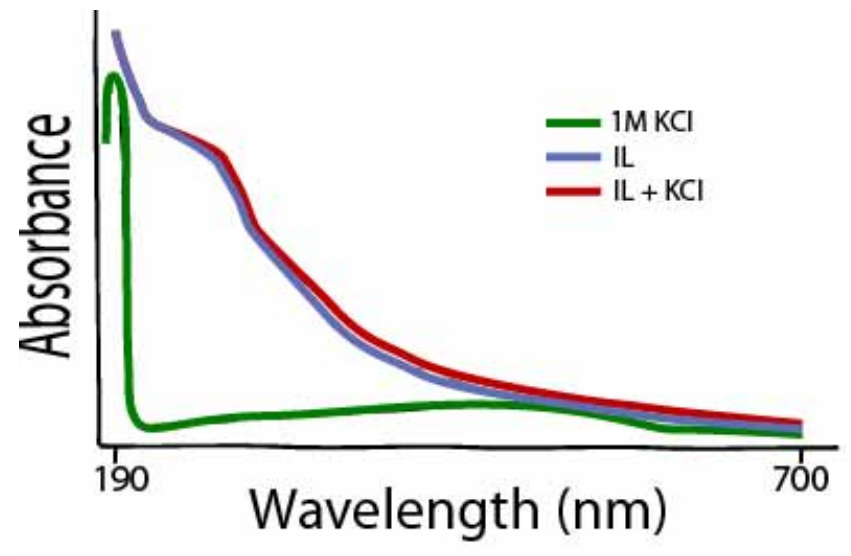

Figure A.3: UV Visible spectroscopy of $1 \mathrm{M} \mathrm{KCl}_{a q}$ (green), $\mathrm{BMIBF}_{4}$ (blue) and $\mathrm{BMIBF}_{4}+\mathrm{KCl}$ (red). It is readily apparent from this graph that the presence of $\mathrm{KCl}$ can not be distinguished from the ionic liquid. Data collected in collaboration with Jerome Ferrance.

\section{A.1.3 Mohr Method}

Potassium chloride salts were finally shown to be present in the ionic liquid via the Mohr method, which is an argentometry technique used to quantify the presence of chloride ions in a solution. The procedure involves the addition of a known molarity of silver nitrate $\left(\mathrm{AgNO}_{3}\right)$ and potassium chromate $\left(\mathrm{K}_{2} \mathrm{CrO}_{4}\right)$ to the test solution. Silver ions then react with any available chloride ions in the sample to produce a silver chloride precipitate $(\mathrm{AgCl}(\mathrm{s})$, see Reaction A.1). When there are no more chloride ions left, excess silver ions react with the available chromate ions to produce a red hued precipitate of silver chromate (see Reaction A.2). The solution changes color as the end point indicator accumulates, allowing the molar concentration of chloride ions to be calculated by stoichiometric equivalence.

$$
\begin{gathered}
\mathrm{Ag}_{(a q)}^{+}+\mathrm{Cl}_{(a q)}^{-} \longrightarrow \mathrm{AgCl}_{(s)} \\
2 \mathrm{Ag}_{(a q)}^{+}+\mathrm{CrO}_{4(a q)}^{2-} \longrightarrow \mathrm{Ag}_{2} \mathrm{CrO}_{4(s)}
\end{gathered}
$$

A calibration curve was first produced using the Mohr method to assertain its detection limit. A range of $\mathrm{KCl}_{a q}$ solutions were produced and tested, from $10 \mu \mathrm{M}$ to $1 \mathrm{M} \mathrm{KCl}$ in $\operatorname{diH}_{2} \mathrm{O}$ (see Figure A.4). The detection limit for the Mohr method was found to be around $1 \mathrm{mM} \mathrm{KCl}$ aq.

The Mohr method was then used to test four samples containing ionic liquid $\left(\mathrm{BMIBF}_{4}\right)$ and either $0.05 \mathrm{M}, 0.1 \mathrm{M}, 0.5 \mathrm{M}$ or $1 \mathrm{M} \mathrm{KCl}$, all of which were well above the detection limit. However, most of these solutions displayed significant $\mathrm{KCl}$ precipitation. Results showed that the saturation of $\mathrm{KCl}$ in ion liquid was lower than the concentrations added to solution (see Figure A.4). The maximum $\mathrm{KCl}$ detected 


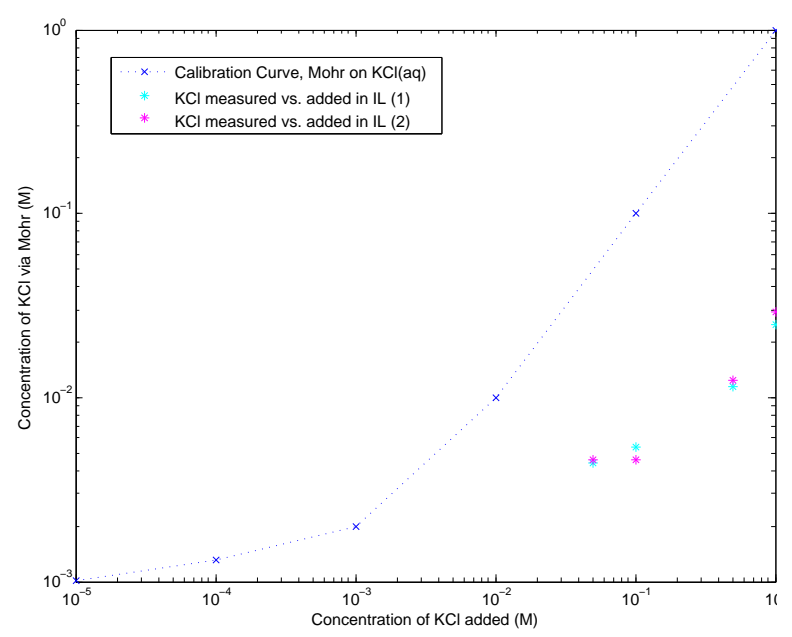

Figure A.4: The Mohr method, which quantifies the presence of $\mathrm{Cl}^{-}$ions in solution, was first used to produce a calibration curve on a range of $\mathrm{KCl}_{a q}$ solutions. It was then used to test samples of the ionic liquid $\mathrm{BMIBF}_{4}$ containing varying concentrations of $\mathrm{KCl}(\mathrm{s})$. A detection limit of $1 \mathrm{mM}$ $\mathrm{KCl}_{a q}$ was found, and a saturation limit within the ionic liquid around $27 \mathrm{mM} \mathrm{KCl}$.

in IL was $27 \mathrm{mM}(\mathrm{n}=2)$ in the case of $1 \mathrm{M} \mathrm{KCl}$ added, indicating a mere $2.7 \%$ salt incorporation.

Since the IL was found to be miscible with water, it was hypothesized that the saturation limit for $\mathrm{KCl}$ within the ILPG could be improved via their combination. A rapid investigation, however, showed the ILPGs resulting from the addition of water to their preparation were poorly formed, containing large open pores where water later evaporated.

In a further attempt to improve the saturation limit of potassium within the IL, a new salt for incorporation was investigated. Work by Giroud et al. showed that an $\mathrm{LiBF}_{4}$ salt could be dissolved in $\mathrm{BMIBF}_{4}$ at concentrations up to 4 molar [203]. Their work inspired the addition of $\mathrm{KBF}_{4}$ as a source for potassium in the ionic liquid. Since the Mohr method detects the presence of $\mathrm{Cl}^{-}$ions only, a new way to probe the samples for the presence of $\mathrm{K}^{+}$was needed.

\section{A.1.4 EIS Probe and Ion Selective Electrodes}

An EIS probe impedance measurement of ionic liquids saturated with $\mathrm{KBF}_{4}$ was performed and compared to control solutions containing $\mathrm{KBF}_{4}$ dissolved in solution. Unfortunately, the EIS probe was unable to distinguish the presence of the added salt in the ionic liquid.

Finally, the ion selective electrode which can detect the presence of potassium ions in solution was also used on varying concentrations of $\mathrm{KBF}_{4}$ in ionic liquid, with a similar negative result. 


\section{A.1.5 Conclusion}

The presence of potassium chloride tracer ions could not be quantified within the ILPG via either mass spectroscopy, or UV-Vis. The Mohr method for chloride detection revealed a very low salt incorporation within the ionic liquid, and therefore the solubility of $\mathrm{KBF}_{4}$ in IL was investigated instead. The presence of $\mathrm{KBF}_{4}$ could neither be determined via EIS probe or ISE. For these reasons, the ILPG was rejected as a potential material for the gel-cell, and hydrogel material chosen instead which was known to uptake large concentrations of electrolyte (of $\mathrm{KCl}$ salts and others).

\section{A.2 Working Electrode Material}

Polypyrrole was prepared on a variety of working electrodes before settling on the stainless steel plates. Initially, depositions were carried out on metal mesh electrodes in order to retain electrodes within the PPy structure during potential cycling for ion transport experiments. However, after several failed experiments on stainless steel and gold meshes, the idea of an integrated mesh was dropped for a free-standing membrane to be placed against a platinum working electrode in the flux cell instead. A summary of the different working electrodes and their respective reasons for failure are covered below.

\section{A.2.1 Stainless Steel Mesh}

Stainless steel (304) mesh was purchased from Unique Wire Weaving Co., Inc. Wire diameter was $0.001 "$, with 400 x 400 mesh size (36\% open area). The mesh was cleaned using ethanol, rinsed in $\mathrm{diH}_{2} \mathrm{O}$ and cut into $2 \mathrm{~cm}$ by $8 \mathrm{~cm}$ rectangles. A portion of the mesh was secured to a copper dipping electrode using double sided copper tape, and covered in insulating paint.

Deposition was initially performed potentiostatically at $+1.2 \mathrm{~V}$ vs. SCE for two hours. This resulted in membranes which appeared to fill in the gaps of the mesh very nicely (see Figure A.5), however upon flux testing they were shown to leak ions passively. Therefore, alternative conditions for polymerization were investigated, and ultimately galvanostatic depositions were carried out a $0.5,1,2$ and $3 \mathrm{~mA} / \mathrm{cm}^{2}$ for 1 to 6 hours total. The best resulting membranes were obtained with $3 \mathrm{~mA}$ galvanostatic depositions for 6 hours. 


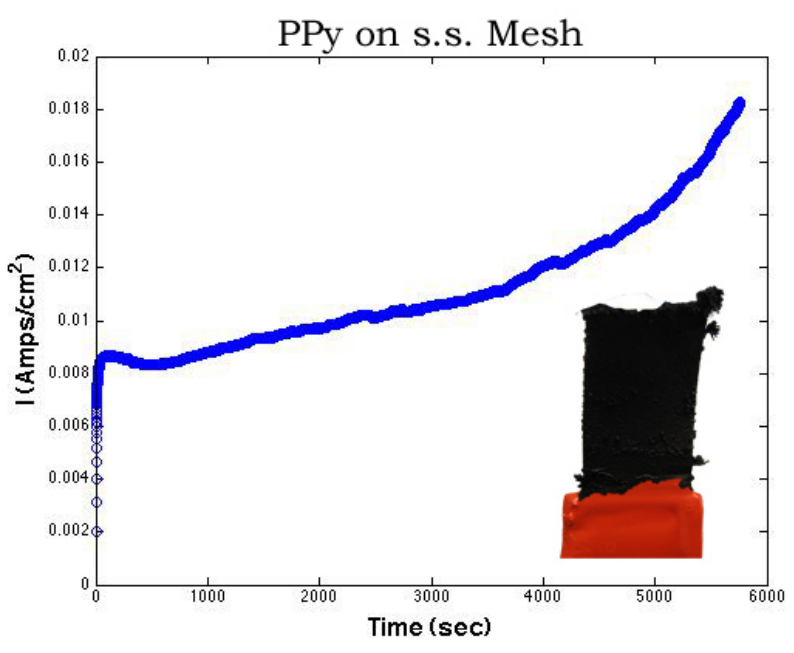

Figure A.5: Chronoamperometric response for a potentiostatic deposition of PPy on stainless steel mesh at $+1.2 \mathrm{~V}$ vs. SCE is shown. An inlay of the resulting PPy membrane is also shown.

In the process of applying $+1 \mathrm{~V}$ vs. SCE to the PPy covered stainless steel mesh in the flux cell, it became apparent that the electrode was corroding from underneath the polymer. In a separate experiment to confirm this, a stainless steel mesh was used as the working electrode in a 3-electrode setup. Using a platinum mesh counter and SCE reference, all three electrodes were placed in an electrolyte solution of 0.1 $\mathrm{M} \mathrm{KCl}_{a q}$ and $+1 \mathrm{~V}$ vs. SCE was applied at the working electrode. This resulted in significant corrosion of the mesh within a few minutes, confirming that stainless steel could not be used in the flux apparatus.

\section{A.2.2 Gold Plated Stainless Steel Mesh}

The stainless steel mesh was then plated with a thin layer of gold in an attempt to protect it from corrosion. Plating was performed using a gold cyanide solution (434 HS RTU, Technic Inc.), a two electrode cell and a voltage source which applied $1.5 \mathrm{~V}$ between a platinum mesh counter electrode and a stainless steel mesh working electrode (see Figure A.6). The plating was performed for varying amounts of time, from 1 to 2 minutes total, to obtain different gold coatings between 400 and $900 \AA$ thick. 


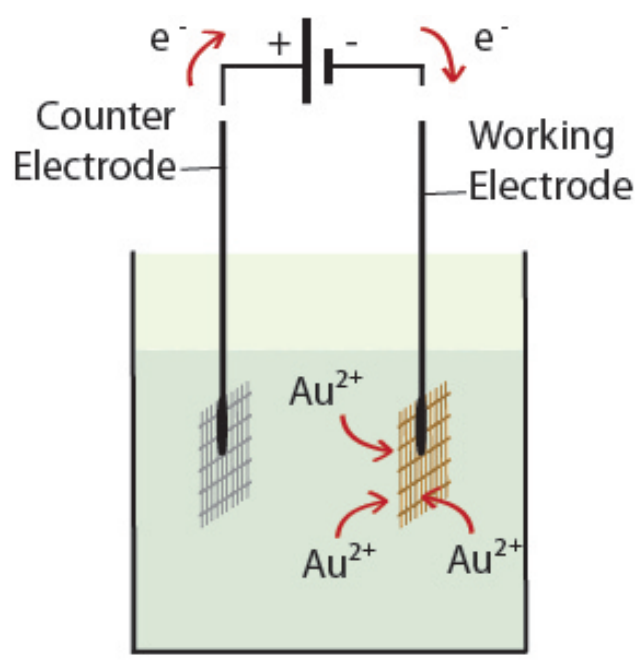

Figure A.6: Gold electroplating was performed by connecting a voltage source across a platinum mesh counter and a stainless steel mesh working electrodes. The electrolyte solution contains gold cyanide, allowing gold ions to plate out onto the cathodic stainless steel mesh.

The resulting gold plated stainless steel mesh electrodes were then tested in a simulation of the redox conditions in the flux cell. A 3-electrode cell was filled with $60 \mathrm{ml}$ of a $0.1 \mathrm{M} \mathrm{KCl}_{a q}$ electrolyte, and $+1 \mathrm{~V}$ vs. SCE was applied to the stainless steel working electrodes for one hour. Below are three gold-plated stainless steel mesh electrodes prepared in this way, before and after reduction. It is apparent that the gold plating was not enough to protect these electrodes from degradation in the setup (see Figure A.7).

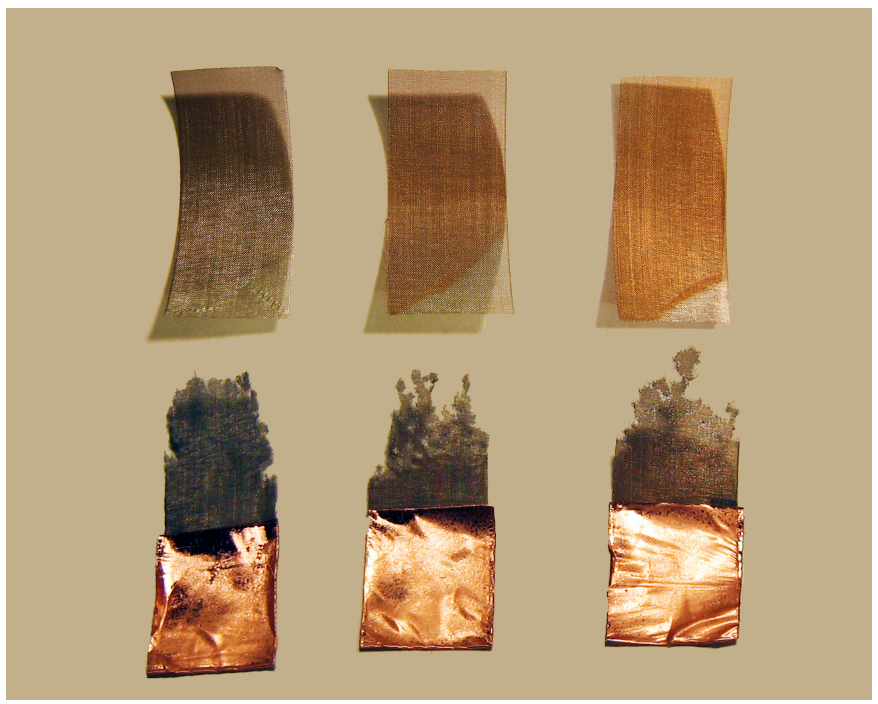

Figure A.7: Three stainless steel steel meshes with increasing gold plate thicknesses from 400 to $800 \AA$ (top, from left to right). The bottom row shows the same mesh electrodes after one hour reduction in potassium chloride electrolyte. 


\section{A.2.3 Gold Mesh}

Gold mesh was purchased from Unique Wire Weaving Co., Inc (www.uniquewire.com). Wire diameter was 0.0025", with $100 \times 100$ mesh size (56\% open area) and 99.99 $\%$ purity. The mesh was cleaned with ethanol, rinsed in $\operatorname{diH}_{2} \mathrm{O}$ and cut into $2 \mathrm{~cm}$ by $8 \mathrm{~cm}$ rectangles prior to use. A portion of the mesh was secured to the copper dipping rods using double sided copper tape, leaving a $2 \mathrm{~cm}$ by $6 \mathrm{~cm}$ area of the mesh extending beyond the electrode, which would be freestanding in the deposition solution. The copper tape and copper dipping electrode were covered in insulating paint (see Figure A.8).

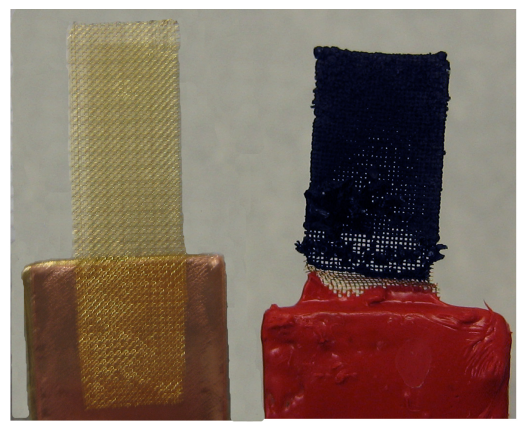

Figure A.8: Gold mesh secured to the copper dipping electrode via double-sided copper tape (left). After deposition, PPy is shown on the gold mesh (right). Pinsize holes still exist in the mesh, however, regardless of deposition conditions.

The deposition was carried out in the same 3-electrode electrochemical cell as mentioned above, with the gold mesh used as working / sense electrode, a saturated calomel reference electrode, and platinum mesh counter electrode. Now using a VersaSTAT 3, potentiostatic depositions were carried out at $+0.8 \mathrm{~V}$ vs. SCE for 1 to up to 6 hours on the gold meshes in an attempt to fill in the pinsize holes left in the PPy depositions. Unfortunately, none of the deposition schemes resulted in water-tight membranes suitable for flux experiments, and it was concluded that the mesh size needed to be reduced significantly. Obtaining a finer gold mesh, however, was cost prohibitive.

\section{A.2.4 Conclusion}

Stainless steel mesh electrodes were used for the electrodeposition of polypyrrole in a water solvent preparation. These electrodes were originally considered to produce PPy and were to be used to apply a current directly to the PPy during flux experiments. However, it was found that the stainless steel material degraded under the voltages applied at the membrane to open and close it.

To resist corrosion, gold-palladium material was electrosputtered onto the surface of the stainless steel mesh electrodes. Unfortunately, the corrosion of the electrodes coated with $\mathrm{Au}-\mathrm{Pd}$ persisted. A gold mesh working electrode was then used for the 
deposition of PPy, however the mesh size was too large and large pores in the resulting membranes lead to leaking in the flux experiments.

Ultimately, stainless steel polished plates were considered and used successfully for the deposition of thin removable PPy membranes which could be placed directly in the flux cell.

\section{A.3 Time-Resolved Hydrogel Flux Experiments}

The time-resolved concentration profile readings for $\mathrm{KCl}_{a q}$ in the receiving solution provided valuable information on the ion transport kinetics through PPy in solution. These experiments were performed by using two potentiostats in parallel within the flux cell. One instrument was used to continuously apply a high frequency alternating current to the EIS probe, while the second potentiostat controlled the potentials applied at the PPy membrane to control its oxidation state.

Unfortunately, in the case of PPy in hydrogel, a feedback mechanism was observed between the two potentiostats in the EIS probe readings. This interference masked the EIS response, making it impossible to quantify the concentration profile of $\mathrm{KCl}$ in the receiving hydrogel.

For this reason, an alternative to the time-resolved EIS capture was established, which allowed for only one potentiostat to be used at one time on the flux cell setup, alternating reading on the EIS and applying a potential at the PPy membrane. This data capture method was referred to as the "time-lapse" data capture method (see Figure A.9). 


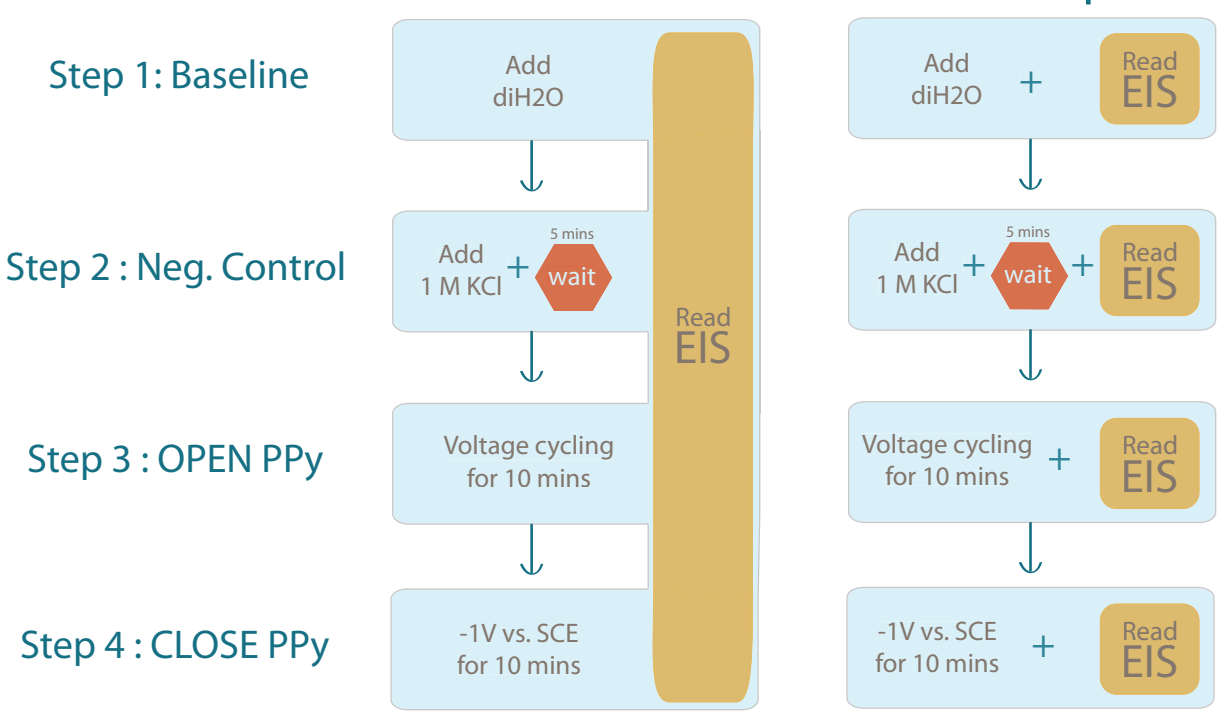

Figure A.9: Flux experiment sequence, shown for both time-resolved and time-lapse capture modes. The two approaches proceed identically through steps 1-4, however in the time-resolved case a continuous read-out of the $\mathrm{KCl}_{a q}$ concentration is provided by the EIS probe, since two potentiostats are used in parallel. In the case of time-lapse measurements, an EIS reading is performed after each step instead, using a single potentiostat. Step 1: a baseline EIS reading is performed on $\operatorname{diH}_{2} \mathrm{O}$. Step 2: a negative control reading is taken after a 5 minute wait post $\mathrm{KCl}_{a q}$ addition. Step 3: the PPy membrane is opened by cycling voltages across it for 10 minutes, Step 4: the membrane is closed. 